\title{
ML(n)BICGSTAB: REFORMULATION, ANALYSIS AND IMPLEMENTATION
}

\author{
MAN-CHUNG YEUNG \\ DEPARTMENT OF MATHEMATICS, UNIVERSITY OF WYOMING, LARAMIE, 82071, USA \\ Dedicated to the Memory of Gene Golub ${ }^{1}$
}

\begin{abstract}
With the aid of index functions, we re-derive the $\operatorname{ML}(n) \operatorname{BiCGStab}$ algorithm in 39 in a more systematic way. It turns out that there are $n$ ways to define the $\operatorname{ML}(n) \operatorname{BiCGStab}$ residual vector. Each definition will lead to a different $\mathrm{ML}(n)$ BiCGStab algorithm. We demonstrate this by presenting a second algorithm which requires less storage. In theory, this second algorithm serves as a bridge that connects the Lanczos-based BiCGStab and the Arnoldi-based FOM while $\mathrm{ML}(n) \mathrm{BiCG}$ a bridge connecting BiCG and FOM. We also analyze the breakdown situations from the probabilistic point of view and summarize some useful properties of $\operatorname{ML}(n) \operatorname{BiCGStab}$. Implementation issues are also addressed.
\end{abstract}

\section{INTRODUCTION}

If we express the BiCG [5, 15] residual as $\mathbf{r}_{k}^{B i C G}=p_{k}(\mathbf{A}) \mathbf{r}_{0}$ in terms of a polynomial, the residual vector $\mathbf{r}_{k}$ of a Lanczos-type product method ${ }^{2}$ based on BiCG is defined to be $\mathbf{r}_{k}=\phi_{k}(\mathbf{A}) p_{k}(\mathbf{A}) \mathbf{r}_{0}$ where $\phi_{k}(\lambda)$ is some polynomial of degree $k$ with $\phi_{k}(0)=1$. In CGS [29], $\phi_{k}=p_{k}$. Since, in every iteration, CGS searches for an approximate solution in a larger Krylov subspace, it often converges much faster than BiCG. However, CGS usually behaves irregularly due to a lack of a smoothing mechanism. In BiCGStab [32], the $\phi_{k}$ is

$$
\phi_{k}(\lambda)= \begin{cases}1 & \text { if } k=0 \\ \left(\rho_{k} \lambda+1\right) \phi_{k-1}(\lambda) & \text { if } k>0 .\end{cases}
$$

Here $\rho_{k}$ is a free parameter selected to minimize the 2-norm of $\mathbf{r}_{k}^{\text {BiCGStab }}$ in the $k$-iteration. As a result, BiCGStab is generally more stable and robust than CGS. BiCGStab has been extended to BiCGStab2 [7] and BiCGStab $(l)$ [24,28] through the use of higher degree minimizing polynomials. In BiCGStab2, the $\phi_{k}$ is defined by the recursion

$$
\phi_{k}(\lambda)= \begin{cases}1 & \text { if } k=0 \\ \left(\rho_{k} \lambda+1\right) \phi_{k-1}(\lambda) & \text { if } k \text { is odd } \\ \left(\left(\alpha_{k} \lambda+\beta_{k}\right)\left(\rho_{k-1} \lambda+1\right)+1-\beta_{k}\right) \phi_{k-2}(\lambda) & \text { if } k \text { is even }\end{cases}
$$

The parameters are again chosen to minimize BiCGStab2 residuals. Likewise, BiCGStab $(l)$ defines its $\phi_{k}$ as

$$
\phi_{k}(\lambda)= \begin{cases}1 & \text { if } k=0 \\ \left(1+\sum_{j=1}^{l} \alpha_{j} \lambda^{j}\right) \phi_{k-l}(\lambda) & \text { if } k \text { is a multiple of } l\end{cases}
$$

where the parameters in the factor $1+\sum_{j=1}^{l} \alpha_{j} \lambda^{j}$ yields an $l$-dimensional minimization in every $l$ th step. BiCGStab2 and BiCGStab $(l)$ usually converge faster than BiCGStab because of smaller

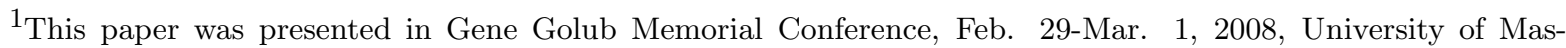
sachusetts, Dartmouth, U.S.A..

${ }^{2}$ For this type of Krylov subspace methods, one can consult 9]. They are called hybrid BiCG methods in 28].
} 
residuals in magnitude while avoiding near-breakdowns caused by a possibly too small $\rho_{k}$. CGS, BiCGStab and BiCGStab2 have been summarized and generalized by GPBi-CG [41]. Here $\phi_{k}$ is

$$
\phi_{k}(\lambda)= \begin{cases}1 & \text { if } k=0 \\ \rho_{1} \lambda+1 & \text { if } k=1 \\ \left(\alpha_{k} \lambda+1+\beta_{k}\right) \phi_{k-1}(\lambda)-\beta_{k} \phi_{k-2}(\lambda) & \text { if } k>1\end{cases}
$$

GPBi-CG will become CGS, BiCGStab or BiCGStab2 when the $\alpha, \beta, \rho$ are appropriately chosen. For detailed descriptions of these and other product-type methods, one is referred to $[6,8,21,23,33$ and the references therein. Moreover, a history of product-type methods can be found in [10]. The history starts three decades ago with IDR [37] method which can be considered as the predecessor of CGS and BiCGStab [25]. Recently, IDR has been generalized to $\operatorname{IDR}(s)$ with a shadow space of higher dimension, see [25, 31, 35]. IDR $(s)$ has close relations with $\operatorname{ML}(s) \operatorname{BiCGStab.}$

Generalizations of BiCGStab to methods based on generalizations of BiCG have been made. For example, BL-BiCGStab [4] is a BiCGStab variant built on the BL-BiCG [17] for the solution of systems with multiple right-hand sides. ML $(n) \operatorname{BiCGStab~39]~is~another~BiCGStab~variant~}$ built on $\mathrm{ML}(n) \mathrm{BiCG}$, a BiCG-like method derived from a variant of the band Lanczos process described in [1] with $n$ left-starting vectors and a single right-starting vector.

The derivation of the $\operatorname{ML}(n)$ BiCGStab algorithm in [39] was complicated. In this paper, we exploit the concept of index functions to re-derive the algorithm in a more systematic way, step by step. Index functions were introduced in [38] by Boley for the purpose of simplifying the development of the transpose-free multiple starting Lanczos process, and they proved to be very helpful.

It turns out that the definition of the $\operatorname{ML}(n) \operatorname{BiCGStab}$ residual vector $\mathbf{r}_{k}$ in [39] is not unique. There are at least $n$ different ways to define $\mathbf{r}_{k}$. Let $\widehat{\mathbf{r}}_{k}$ be the residual of $\operatorname{ML}(n) \operatorname{BiCG}$ and $\phi_{k}(\lambda)$ as in (1.1). Then, the ML(n)BiCGStab residual $\mathbf{r}_{k}$ in [39] is defined as

$$
\mathbf{r}_{k}=\phi_{j}(\mathbf{A}) \widehat{\mathbf{r}}_{k}
$$

where $k=j n+i, 1 \leq i \leq n, j=0,1,2, \cdots$. Starting from $k=1$, let us call every $n$ consecutive iterations an iteration "cycle". For example, iterations $k=1,2, \cdots, n$ form the first cycle, iterations $k=n+1, n+2, \cdots, 2 n$ the second cycle and so on. Then definition (1.2) increases the degree of $\phi$ by 1 at the beginning of a cycle. One actually can define $\mathbf{r}_{k}$ by increasing the degree of $\phi$ by 1 anywhere within an iteration cycle. Each definition will lead to a different $\operatorname{ML}(n) \operatorname{BiCGStab}$ algorithm. As an illustration, we derive a second ML $(n)$ BiCGStab algorithm associated with the definition

$$
\mathbf{r}_{j n+i}= \begin{cases}\phi_{j}(\mathbf{A}) \widehat{\mathbf{r}}_{j n+i} & \text { if } 1 \leq i \leq n-1 \\ \phi_{j+1}(\mathbf{A}) \widehat{\mathbf{r}}_{j n+i} & \text { if } i=n\end{cases}
$$

(1.3) increases the degree of $\phi$ by 1 at the end of a cycle. The resulting algorithm requires about $25 \%$ less storage (not counting the storage of the coefficient matrix and the preconditioner) than the algorithm associated with definition (1.2). However, one drawback with this storage-saving algorithm is that, in some experiments, its computed residual $\mathbf{r}_{k}$ can easily diverge from the corresponding exact residual when $n$ is moderately large.

Both ML $(n)$ BiCG and ML $(n)$ BiCGStab possess a set of left starting vectors (or, shadow vectors) $\mathbf{q}_{1}, \cdots, \mathbf{q}_{n}$ that can be chosen freely. This freedom appears to be an advantage of the methods. It not only helps stabilize the performance of the algorithms, but also allows to see a connection between the Lanczos-based BiCG/BiCGStab and the Arnoldi-based FOM.

Just like BiCGStab, ML $(n)$ BiCGStab can suffer from three types of breakdown, caused respectively by the failure of the underlying Lanczos process, the non-existence of the $L U$ factorization during the construction of $\mathrm{ML}(n) \mathrm{BiCG}$ and the parameters $\rho_{k}$. We prove that the breakdown probability is zero when the shadow vectors are selected randomly. 
The outline of the paper is as follows. In 92 , we introduce index functions. In $₫ 3$, we present the $\mathrm{ML}(n) \mathrm{BiCG}$ algorithm introduced in 39], from which ML $(n)$ BiCGStab algorithms are derived. In 44, we rederive the $\mathrm{ML}(n)$ BiCGStab algorithm in [39] by index functions. In \$5, we derive a storage-saving $\operatorname{ML}(n)$ BiCGStab algorithm from a different definition of the residual vector. In 86. we discuss relationships of $\operatorname{ML}(n)$ BiCGStab with some other methods. In \$7, implementation issues are addressed. Concluding remarks are made in $₫ 8$.

\section{IndEX FunCTIONS}

Let be given a positive integer $n$. For all integers $k$, we define

$$
g_{n}(k)=\lfloor(k-1) / n\rfloor \text { and } r_{n}(k)=k-n g_{n}(k)
$$

where $\lfloor\cdot\rfloor$ rounds its argument to the nearest integer towards minus infinity. We call $g_{n}$ and $r_{n}$ index functions; they are defined on $\mathcal{Z}$, the set of all integers, with ranges $\mathcal{Z}$ and $\{1,2, \cdots, n\}$, respectively.

If we write

$$
k=j n+i
$$

with $1 \leq i \leq n$ and $j \in \mathcal{Z}$, then

$$
g_{n}(j n+i)=j \quad \text { and } \quad r_{n}(j n+i)=i .
$$

\begin{tabular}{|c|c|c|c|c|c|c|}
\hline$k$ & 0 & 123 & 456 & 789 & 101112 & \\
\hline$g_{n}(k)$ & -1 & 000 & 111 & 222 & 333 & $\cdots$ \\
\hline$r_{n}(k)$ & 3 & 123 & 123 & 123 & 123 & $\cdots$ \\
\hline
\end{tabular}

Table 2.1 illustrates the behavior of $g_{n}$ and $r_{n}$ with $n=3$. It can be seen that $g_{n}(k)$ has a jump when $k$, moved from left to right, passes a multiple of $n$.

TABLE 2.1. Simple illustration of the index functions for $n=3$.

The following properties can be easily verified by using (2.2) .

Proposition 2.1. Let $k \in \mathcal{N}$, the set of all positive integers, and $s \in \mathcal{N}_{0}:=\mathcal{N} \cup\{0\}$.

(a) $g_{n}(k+n)=g_{n}(k)+1$ and $r_{n}(k+n)=r_{n}(k)$.

(b) $g_{n}(s+1)+1=g_{n}(k+1)$ if $\max (k-n, 0) \leq s \leq g_{n}(k) n-1$.

(c) $g_{n}(s+1)=g_{n}\left(g_{n}(k) n+1\right)=g_{n}(k)$ if $g_{n}(k) n \leq s \leq k-1$.

(d) $g_{n}(k+1)=g_{n}(k)+1$ if $r_{n}(k)=n$. $g_{n}(k+1)=g_{n}(k)$ if $r_{n}(k)<n$.

(e) $\max (k-n, 0)>g_{n}(k) n-1$ if $r_{n}(k)=n$ or $g_{n}(k)=0$.

\section{3. $\mathrm{A} \operatorname{ML}(n) \mathrm{BiCG}$ Algorithm}

Parallel to the derivation of BiCGStab from BiCG, ML $(n)$ BiCGStab was derived in [39] from a BiCG-like method named $\mathrm{ML}(n) \mathrm{BiCG}$, which was built upon a band Lanczos process with $n$ left starting vectors and a single right starting vector. In this section, we present the algorithm of $\mathrm{ML}(n) \mathrm{BiCG}$ from [39] and summarize some properties of it. 
3.1. The Algorithm. Consider the solution of the linear system

$$
\mathbf{A x}=\mathbf{b}
$$

where $\mathbf{A} \in \mathcal{C}^{N \times N}$ and $\mathbf{b} \in \mathcal{C}^{N}$. Throughout the paper we do not assume the matrix $\mathbf{A}$ is nonsingular except where specified. The solution of singular systems has been extensively studied in area of iterative methods, see, for instance, [2, 14, 16, 19, 36, 40].

Let be given $n$ vectors $\mathbf{q}_{1}, \ldots, \mathbf{q}_{n} \in \mathcal{C}^{N}$, which we call left starting vectors or shadow vectors. Set

$$
\mathbf{p}_{k}=\left(\mathbf{A}^{H}\right)^{g_{n}(k)} \mathbf{q}_{r_{n}(k)}
$$

for $k=1,2, \cdots$. The following algorithm for solving (3.1) is from [39].

\section{Algorithm 3.1. $\mathrm{ML}(n) \mathrm{BiCG}^{3}$}

1. Choose an initial guess $\widehat{\mathbf{x}}_{0}$ and $n$ vectors $\mathbf{q}_{1}, \mathbf{q}_{2}, \cdots, \mathbf{q}_{n}$.

2. Compute $\widehat{\mathbf{r}}_{0}=\mathbf{b}-\mathbf{A} \widehat{\mathbf{x}}_{0}$ and set $\mathbf{p}_{1}=\mathbf{q}_{1}, \widehat{\mathbf{g}}_{0}=\widehat{\mathbf{r}}_{0}$.

3. For $k=1,2, \cdots$, until convergence:

4. $\alpha_{k}=\mathbf{p}_{k}^{H} \widehat{\mathbf{r}}_{k-1} / \mathbf{p}_{k}^{H} \mathbf{A} \widehat{\mathbf{g}}_{k-1}$;

5. $\quad \widehat{\mathbf{x}}_{k}=\widehat{\mathbf{x}}_{k-1}+\alpha_{k} \widehat{\mathbf{g}}_{k-1}$;

6. $\quad \widehat{\mathbf{r}}_{k}=\widehat{\mathbf{r}}_{k-1}-\alpha_{k} \mathbf{A} \widehat{\mathbf{g}}_{k-1}$;

7. For $s=\max (k-n, 0), \cdots, k-1$

8. $\quad \beta_{s}^{(k)}=-\mathbf{p}_{s+1}^{H} \mathbf{A}\left(\widehat{\mathbf{r}}_{k}+\sum_{t=\max (k-n, 0)}^{s-1} \beta_{t}^{(k)} \widehat{\mathbf{g}}_{t}\right) / \mathbf{p}_{s+1}^{H} \mathbf{A} \widehat{\mathbf{g}}_{s} ;$

9. End

10. $\widehat{\mathbf{g}}_{k}=\widehat{\mathbf{r}}_{k}+\sum_{s=\max (k-n, 0)}^{k-1} \beta_{s}^{(k)} \widehat{\mathbf{g}}_{s}$

11. Compute $\mathbf{p}_{k+1}$ according to (3.2)

12. End

The $\mathrm{ML}(n) \mathrm{BiCG}$ algorithm is a variation of the classical BiCG algorithm. The left-hand side (shadow) Krylov subspace of BiCG is replaced by the block Krylov subspace with $n$ starting vectors $\mathbf{q}_{1}, \mathbf{q}_{2}, \cdots, \mathbf{q}_{n}$ :

$$
\begin{aligned}
\mathcal{B}_{k} & :=\text { the space spanned by the first } k \text { columns of }\left[\mathbf{Q}, \mathbf{A}^{H} \mathbf{Q},\left(\mathbf{A}^{H}\right)^{2} \mathbf{Q}, \cdots\right] \\
& =\operatorname{span}\left\{\mathbf{p}_{1}, \mathbf{p}_{2}, \cdots, \mathbf{p}_{k}\right\} \\
& =\sum_{i=1}^{r_{n}(k)} \mathcal{K}_{g_{n}(k)+1}\left(\mathbf{A}^{H}, \mathbf{q}_{i}\right)+\sum_{i=r_{n}(k)+1}^{n} \mathcal{K}_{g_{n}(k)}\left(\mathbf{A}^{H}, \mathbf{q}_{i}\right)
\end{aligned}
$$

where $\mathbf{Q}:=\left[\mathbf{q}_{1}, \mathbf{q}_{2}, \cdots, \mathbf{q}_{n}\right]$ and

$$
\mathcal{K}_{t}(\mathbf{M}, \mathbf{v}):=\operatorname{span}\left\{\mathbf{v}, \mathbf{M v}, \cdots, \mathbf{M}^{t-1} \mathbf{v}\right\}
$$

for $\mathbf{M} \in \mathcal{C}^{N \times N}, \mathbf{v} \in \mathcal{C}^{N}$ and $t \in \mathcal{N}$. Moreover, in $\mathrm{ML}(n) \mathrm{BiCG}$, the basis used for $\mathcal{B}_{k}$ is not chosen to be bi-orthogonal, but simply the set $\left\{\mathbf{p}_{1}, \mathbf{p}_{2}, \cdots, \mathbf{p}_{k}\right\}$. Therefore, the ML $(n) \operatorname{BiCG}$ algorithm can be viewed as a generalization of a one-sided Lanczos algorithm (see [9,20]). The likely illconditioning of this basis does not matter, as the algorithm is only a technical tool for deriving $\operatorname{ML}(n)$ BiCGStab and this basis disappears in ML( $n)$ BiCGStab because $\mathbf{A}^{H}$ will be absorbed by the residuals and direction vectors of $\operatorname{ML}(n)$ BiCGStab. For constructing the right-hand side basis consisting of residuals $\widehat{\mathbf{r}}_{k}$, we used recurrences that generalize the coupled two-term recurrences of $\mathrm{BiCG}$, that is, direction vectors $\widehat{\mathbf{g}}_{k}$ are also constructed.

\footnotetext{
${ }^{3}$ Algorithm 3.1 consists of exact mathematical formulas for $\alpha_{k}, \beta_{s}^{(k)}, \widehat{\mathbf{x}}_{k}, \widehat{\mathbf{r}}_{k}$ and $\widehat{\mathbf{g}}_{k}$ obtained in $\S 3$ of [39]. Repeated operations should be removed in order to make the algorithm computationally efficient. Moreover, even though the algorithm has not been tested, it is believed to be numerically instable because of Line 11 in which the left starting vectors are repeatedly multiplied by $\mathbf{A}^{H}$, a type of operation which is highly sensitive to round-off errors. The algorithm has been introduced only for the purpose of developing $\mathrm{ML}(n) \operatorname{BiCGStab}$ algorithms.
} 
3.2. Properties. Let $\nu$ be the degree of the minimal polynomial $p_{\min }\left(\lambda ; \mathbf{A}, \widehat{\mathbf{r}}_{0}\right)$ of $\widehat{\mathbf{r}}_{0}$ with respect to $\mathbf{A}$ (that is, the unique monic polynomial $p(\lambda)$ of minimum degree such that $p(\mathbf{A}) \widehat{\mathbf{r}}_{0}=\mathbf{0}$ ) and let

$$
\widehat{\mathbf{S}}_{\nu}=\left[\mathbf{p}_{1}, \mathbf{p}_{2}, \cdots, \mathbf{p}_{\nu}\right]^{H} \mathbf{A}\left[\widehat{\mathbf{r}}_{0}, \mathbf{A} \widehat{\mathbf{r}}_{0}, \cdots, \mathbf{A}^{\nu-1} \widehat{\mathbf{r}}_{0}\right]
$$

and

$$
\widehat{\mathbf{W}}_{\nu}=\left[\mathbf{p}_{1}, \mathbf{p}_{2}, \cdots, \mathbf{p}_{\nu}\right]^{H}\left[\widehat{\mathbf{r}}_{0}, \mathbf{A} \widehat{\mathbf{r}}_{0}, \cdots, \mathbf{A}^{\nu-1} \widehat{\mathbf{r}}_{0}\right]
$$

Denote by $\widehat{\mathbf{S}}_{l}$ and $\widehat{\mathbf{W}}_{l}$ the $l \times l$ leading principal submatrices of $\widehat{\mathbf{S}}_{\nu}$ and $\widehat{\mathbf{W}}_{\nu}$ respectively. We now summarize some useful facts about Algorithm 3.1. They can be derived from the construction procedure of the algorithm.

Proposition 3.1. In infinite precision arithmetic, if $\prod_{l=1}^{\nu} \operatorname{det}\left(\widehat{\mathbf{S}}_{l}\right) \operatorname{det}\left(\widehat{\mathbf{W}}_{l}\right) \neq 0$, then Algorithm 3.1 does not break down by zero division for $k=1,2, \cdots, \nu$, and $x_{\nu}$ is the exact solution of (3.1). Moreover, the computed quantities satisfy

(a) $\widehat{\mathbf{x}}_{k} \in \widehat{\mathbf{x}}_{0}+\mathcal{K}_{k}\left(\mathbf{A}, \widehat{\mathbf{r}}_{0}\right)$ and $\widehat{\mathbf{r}}_{k}=\mathbf{b}-\mathbf{A} \widehat{\mathbf{x}}_{k} \in \widehat{\mathbf{r}}_{0}+\mathbf{A} \mathcal{K}_{k}\left(\mathbf{A}, \widehat{\mathbf{r}}_{0}\right)$ for $1 \leq k \leq \nu$.

(b) $\operatorname{span}\left\{\widehat{\mathbf{r}}_{0}, \widehat{\mathbf{r}}_{1}, \cdots, \widehat{\mathbf{r}}_{k-1}\right\}=\mathcal{K}_{k}\left(\mathbf{A}, \widehat{\mathbf{r}}_{0}\right)$ for $1 \leq k \leq \nu$.

(c) $\operatorname{span}\left\{\mathbf{A} \widehat{\mathbf{r}}_{0}, \mathbf{A} \widehat{\mathbf{r}}_{1}, \cdots, \mathbf{A} \widehat{\mathbf{r}}_{\nu-1}\right\}=\mathcal{K}_{\nu}\left(\mathbf{A}, \widehat{\mathbf{r}}_{0}\right)$.

(d) $\widehat{\mathbf{r}}_{k} \perp \mathcal{B}_{k}$ and $\widehat{\mathbf{r}}_{k} \not \perp \mathbf{p}_{k+1}$ for $0 \leq k \leq \nu-1{ }^{4}$

(e) $\operatorname{span}\left\{\widehat{\mathbf{g}}_{0}, \widehat{\mathbf{g}}_{1}, \cdots, \widehat{\mathbf{g}}_{k-1}\right\}=\mathcal{K}_{k}\left(\mathbf{A}, \widehat{\mathbf{r}}_{0}\right)$ for $1 \leq k \leq \nu$.

(f) $\operatorname{span}\left\{\mathbf{A} \widehat{\mathbf{g}}_{0}, \mathbf{A} \widehat{\mathbf{g}}_{1}, \cdots, \mathbf{A} \widehat{\mathbf{g}}_{\nu-1}\right\}=\mathcal{K}_{\nu}\left(\mathbf{A}, \widehat{\mathbf{r}}_{0}\right)$.

(g) $\mathbf{A} \widehat{\mathbf{g}}_{k} \perp \mathcal{B}_{k}$ and $\mathbf{A} \widehat{\mathbf{g}}_{k} \not \perp \mathbf{p}_{k+1}$ for $0 \leq k \leq \nu-1$.

Because of Proposition 3.1(a) and (d), ML(n)BiCG is an oblique projection Krylov subspace method [21].

Remarks:

(i) The matrices $\widehat{\mathbf{S}}_{l}$ and $\widehat{\mathbf{W}}_{l}$ have already appeared in [12,13] where they were called moment matrices. Proposition 3.1 can be regarded as a generalization of Theorem 2 in [13] from $n=1$ to $n>1$.

(ii) Just like BiCG, ML $(n) \mathrm{BiCG}$ also has two types of breakdown caused, respectively, by the failure of the underlying Lanczos process and the nonexistence of the $L U$ factorizations of the Hessenberg matrix of the recurrence coefficients. Both types of breakdown are reflected in Algorithm 3.1 by $\mathbf{p}_{k}^{H} \mathbf{A} \widehat{\mathbf{g}}_{k-1}=0$. The condition $\prod_{l=1}^{\nu} \operatorname{det}\left(\widehat{\mathbf{W}}_{l}\right) \neq 0$ guarantees that the underlying Lanczos process works without breakdown, and the condition $\prod_{l=1}^{\nu} \operatorname{det}\left(\widehat{\mathbf{S}}_{l}\right) \neq 0$ ensures that the $L U$ factorizations exist.

(iii) $\operatorname{det}\left(\widehat{\mathbf{S}}_{\nu}\right) \neq 0$ implies that $p_{\min }\left(0 ; \mathbf{A}, \widehat{\mathbf{r}}_{0}\right) \neq 0$ which, in turn, implies that (3.1) is consistent and has a solution lying in $\widehat{\mathbf{x}}_{0}+\mathcal{K}_{\nu}\left(\mathbf{A}, \widehat{\mathbf{r}}_{0}\right)$.

The derivation of $\mathrm{ML}(n)$ BiCGStab will require the following result which, in the case when $n=1$, has been used in CGS and BiCGStab.

Corollary 3.1. Let $s \in \mathcal{N}$ and

$$
\psi_{g_{n}(s)}(\lambda)=c_{g_{n}(s)} \lambda^{g_{n}(s)}+c_{g_{n}(s)-1} \lambda^{g_{n}(s)-1}+\cdots+c_{0}
$$

be any polynomial of exact degree $g_{n}(s)$. Then, under the assumptions of Proposition 3.1,

$$
\mathbf{p}_{s}^{H} \widehat{\mathbf{r}}_{k}=\frac{1}{c_{g_{n}(s)}} \mathbf{q}_{r_{n}(s)}^{H} \psi_{g_{n}(s)}(\mathbf{A}) \widehat{\mathbf{r}}_{k} \quad \text { and } \quad \mathbf{p}_{s}^{H} \mathbf{A} \widehat{\mathbf{g}}_{k}=\frac{1}{c_{g_{n}(s)}} \mathbf{q}_{r_{n}(s)}^{H} \mathbf{A} \psi_{g_{n}(s)}(\mathbf{A}) \widehat{\mathbf{g}}_{k}
$$

if $0 \leq k \leq \nu-1$ and $s \leq k+n$.

\footnotetext{
${ }^{4}$ We say that $\mathbf{u} \perp \mathbf{v}$ if $\mathbf{u}^{H} \mathbf{v}=0$.
} 
Proof. It is easy to verify that

$$
\mathbf{p}_{s}-\frac{1}{\bar{c}_{g_{n}(s)}} \bar{\psi}_{g_{n}(s)}\left(\mathbf{A}^{H}\right) \mathbf{q}_{r_{n}(s)} \in \mathcal{B}_{k}
$$

by Proposition 2.1(a) and (3.2), where the overbar denotes complex conjugation. The corollary then follows from Proposition 3.1(d) and (g).

Corollary 3.1 essentially says that adding to $\mathbf{p}_{s}$ a vector from $\mathcal{B}_{k}$ does not change the inner products $\mathbf{p}_{s}^{H} \widehat{\mathbf{r}}_{k}$ and $\mathbf{p}_{s}^{H} \mathbf{A} \widehat{\mathbf{g}}_{k}$.

Examples exist where the condition $\prod_{l=1}^{\nu} \operatorname{det}\left(\widehat{\mathbf{W}}_{l}\right) \operatorname{det}\left(\widehat{\mathbf{S}}_{l}\right) \neq 0$ in Proposition 3.1 holds, as shown below.

Lemma 3.1. Consider the case where $n=1, \widehat{\mathbf{r}}_{0} \in \mathcal{R}^{N}, \widehat{\mathbf{r}}_{0} \neq \mathbf{0}^{5}$ and $\mathbf{A} \in \mathcal{R}^{N \times N}$ is nonsingular. If $\mathbf{q}_{1} \in \mathcal{R}^{N}$ is a random vector with independent and identically distributed elements from $N(0,1)$, the normal distribution with mean 0 and variance 1 , then $\operatorname{Prob}\left(\prod_{l=1}^{\nu} \operatorname{det}\left(\widehat{\mathbf{W}}_{l}\right) \operatorname{det}\left(\widehat{\mathbf{S}}_{l}\right)=0\right)=0$.

Proof. Since $\mathbf{p}_{k}=\mathbf{A}^{g_{n}(k)} \mathbf{q}_{r_{n}(k)}=\mathbf{A}^{k-1} \mathbf{q}_{1}$ when $n=1$, both $\widehat{\mathbf{S}}_{\nu}$ and $\widehat{\mathbf{W}}_{\nu}$ are Hankel matrices

$$
\widehat{\mathbf{S}}_{\nu}=\left[\begin{array}{llll}
\widehat{s}_{1} & \widehat{s}_{2} & \cdots & \widehat{s}_{\nu} \\
\widehat{s}_{2} & \widehat{s}_{3} & \cdots & \widehat{s}_{\nu+1} \\
\cdots & \cdots & \cdots & \cdots \\
\widehat{s}_{\nu} & \widehat{s}_{\nu+1} & \cdots & \widehat{s}_{2 \nu-1}
\end{array}\right], \quad \widehat{\mathbf{W}}_{\nu}=\left[\begin{array}{llll}
\widehat{w}_{1} & \widehat{w}_{2} & \cdots & \widehat{w}_{\nu} \\
\widehat{w}_{2} & \widehat{w}_{3} & \cdots & \widehat{w}_{\nu+1} \\
\cdots & \cdots & \cdots & \cdots \\
\widehat{w}_{\nu} & \widehat{w}_{\nu+1} & \cdots & \widehat{w}_{2 \nu-1}
\end{array}\right]
$$

where $\widehat{s}_{t}=\mathbf{q}_{1}^{T} \mathbf{A}^{t} \widehat{\mathbf{r}}_{0}$ and $\widehat{w}_{t}=\mathbf{q}_{1}^{T} \mathbf{A}^{t-1} \widehat{\mathbf{r}}_{0}$ for $t=1,2, \cdots, 2 \nu-1$.

We first prove

$$
\operatorname{Prob}\left(\operatorname{det}\left(\widehat{\mathbf{W}}_{l}\right)=0\right)=0
$$

for any fixed $l$ with $1 \leq l \leq \nu$. It is trivial that (3.3) holds when $l=1$ and we therefore assume $l \geq 2$ in the following discussion.

By assumption, $\nu$ is the degree of the minimal polynomial of $\widehat{\mathbf{r}}_{0}$ with respect to $\mathbf{A}$. This implies that $\mathcal{K}:=\operatorname{span}\left\{\mathbf{A}^{t} \widehat{\mathbf{r}}_{0} \mid t \in \mathcal{N}_{0}\right\}$ is a $\nu$-dimensional space with $\left\{\widehat{\mathbf{r}}_{0}, \mathbf{A} \widehat{\mathbf{r}}_{0}, \cdots, \mathbf{A}^{\nu-1} \widehat{\mathbf{r}}_{0}\right\}$ as a basis. Since $\mathbf{A}$ is nonsingular, $\left\{\mathbf{A}^{l-1} \widehat{\mathbf{r}}_{0}, \mathbf{A}^{l} \widehat{\mathbf{r}}_{0}, \cdots, \mathbf{A}^{l+\nu-2} \widehat{\mathbf{r}}_{0}\right\}$ is another basis of $\mathcal{K}$.

Perform an orthogonal factorization of the $N \times \nu$ matrix

$$
\left[\mathbf{A}^{l-1} \widehat{\mathbf{r}}_{0}, \mathbf{A}^{l} \widehat{\mathbf{r}}_{0}, \cdots, \mathbf{A}^{l+\nu-2} \widehat{\mathbf{r}}_{0}\right]=\mathbf{Q R}
$$

where $\mathbf{Q} \in \mathcal{R}^{N \times N}$ is orthogonal and $\mathbf{R} \in \mathcal{R}^{N \times \nu}$ is upper triangular with positive main diagonal elements $r_{11}, r_{22}, \cdots, r_{\nu \nu}$. Clearly, the first $\nu$ columns of $\mathbf{Q}$ form a basis of $\mathcal{K}$ and the last $N-\nu$ columns belong to $\mathcal{K}^{\perp}$.

Write

$$
\begin{aligned}
\mathbf{A}^{l-2} \widehat{\mathbf{r}}_{0} & =\xi_{1} \mathbf{A}^{l-1} \widehat{\mathbf{r}}_{0}+\xi_{2} \mathbf{A}^{l} \widehat{\mathbf{r}}_{0}+\cdots+\xi_{\nu} \mathbf{A}^{l+\nu-2} \widehat{\mathbf{r}}_{0} \\
& =\left[\mathbf{A}^{l-1} \widehat{\mathbf{r}}_{0}, \mathbf{A}^{l} \widehat{\mathbf{r}}_{0}, \cdots, \mathbf{A}^{l+\nu-2} \widehat{\mathbf{r}}_{0}\right] \xi \\
& =\mathbf{Q R} \xi \equiv \mathbf{Q} \eta
\end{aligned}
$$

for some scalars $\xi_{1}, \xi_{2}, \cdots, \xi_{\nu} \in \mathcal{R}$, where $\xi=\left[\xi_{1}, \xi_{2}, \cdots, \xi_{\nu}\right]^{T} \in \mathcal{R}^{\nu}$ and $\eta=\left[\eta_{1}, \eta_{2}, \cdots, \eta_{N}\right]^{T}=$ $\mathbf{R} \xi \in \mathcal{R}^{N}$. Since $\mathbf{A}$ is nonsingular and $\left\{\widehat{\mathbf{r}}_{0}, \mathbf{A} \widehat{\mathbf{r}}_{0}, \cdots, \mathbf{A}^{\nu-1} \widehat{\mathbf{r}}_{0}\right\}$ linearly independent, we have $\xi_{\nu} \neq 0$ and hence $\eta_{\nu}=r_{\nu \nu} \xi_{\nu} \neq 0$. Let $\theta=\left[\theta_{1}, \theta_{2}, \cdots, \theta_{N}\right]^{T}=\mathbf{Q}^{T} \mathbf{q}_{1}$. Then $\theta$ is a random vector

\footnotetext{
${ }^{5}$ In Lemma 3.1 and Theorem 3.1, $\widehat{\mathbf{r}}_{0}$ can be any non-zero vector in $\mathcal{R}^{N}$, not necessary to be a residual vector like $\widehat{\mathbf{r}}_{0}=\mathbf{b}-\mathbf{A} \widehat{\mathbf{x}}_{0}$.
} 
with iid elements from $N(0,1)[3]$. We now express $\operatorname{det}\left(\widehat{\mathbf{W}}_{l}\right)$ in terms of the elements of $\theta$. Let us write

$$
\begin{aligned}
& {\left[\widehat{w}_{1}, \widehat{w}_{2}, \cdots, \widehat{w}_{l-2}, \widehat{w}_{l-1}, \widehat{w}_{l}, \cdots, \widehat{w}_{2 l-1}\right] } \\
= & \mathbf{q}_{1}^{T}\left[\widehat{\mathbf{r}}_{0}, \mathbf{A} \widehat{\mathbf{r}}_{0}, \cdots, \mathbf{A}^{l-3} \widehat{\mathbf{r}}_{0}, \mathbf{A}^{l-2} \widehat{\mathbf{r}}_{0}, \mathbf{A}^{l-1} \widehat{\mathbf{r}}_{0}, \cdots, \mathbf{A}^{2 l-2} \widehat{\mathbf{r}}_{0}\right] \\
= & \mathbf{q}_{1}^{T}\left[\widehat{\mathbf{r}}_{0}, \mathbf{A} \widehat{\mathbf{r}}_{0}, \cdots, \mathbf{A}^{l-3} \widehat{\mathbf{r}}_{0}, \mathbf{Q} \eta, \mathbf{Q R} \mathbf{R}^{(l)}\right] \\
= & \mathbf{q}_{1}^{T} \mathbf{Q}\left[\mathbf{Q}^{T}\left[\widehat{\mathbf{r}}_{0}, \mathbf{A} \widehat{\mathbf{r}}_{0}, \cdots, \mathbf{A}^{l-3} \widehat{\mathbf{r}}_{0}\right], \eta, \mathbf{R}^{(l)}\right] \\
= & \theta^{T}\left[\mathbf{Q}^{T}\left[\widehat{\mathbf{r}}_{0}, \mathbf{A} \widehat{\mathbf{r}}_{0}, \cdots, \mathbf{A}^{l-3} \widehat{\mathbf{r}}_{0}\right], \eta, \mathbf{R}^{(l)}\right]
\end{aligned}
$$

where $\mathbf{R}^{(l)}$ denotes the matrix consisting of the first $l$ columns of $\mathbf{R}$. Since the last $N-\nu$ columns of $\mathbf{Q}$ belong to $\mathcal{K}^{\perp}$, the last $N-\nu$ rows of the matrix $\mathbf{Q}^{T}\left[\widehat{\mathbf{r}}_{0}, \mathbf{A} \widehat{\mathbf{r}}_{0}, \cdots, \mathbf{A}^{l-3} \widehat{\mathbf{r}}_{0}\right]$ are zeros. Similarly, the last $N-\nu$ elements of $\eta=\mathbf{R} \xi$ are zeros because the last $N-\nu$ rows of $\mathbf{R}$ are zeros. We therefore have

$$
\widehat{w}_{t}=\left\{\begin{array}{c}
\text { a linear combination of } \theta_{1}, \theta_{2}, \cdots, \theta_{\nu} \text { if } 1 \leq t \leq l-2, \\
\eta_{1} \theta_{1}+\eta_{2} \theta_{2}+\cdots+\eta_{\nu} \theta_{\nu} \text { with } \eta_{\nu} \neq 0 \text { if } t=l-1 \\
r_{1, t-l+1} \theta_{1}+r_{2, t-l+1} \theta_{2}+\cdots+r_{t-l+1, t-l+1} \theta_{t-l+1} \\
\text { with } r_{t-l+1, t-l+1} \neq 0 \text { if } l \leq t \leq 2 l-1
\end{array}\right.
$$

This shows that none of the random variables $\theta_{\nu+1}, \theta_{\nu+2}, \cdots, \theta_{N}$ is involved in any of the $\widehat{w}$ 's. In more detail, when $l<\nu, \widehat{w}_{t}=\widehat{w}_{t}\left(\theta_{1}, \theta_{2}, \cdots, \theta_{\nu}\right)$ if $1 \leq t \leq l-1$ and $\widehat{w}_{t}=\widehat{w}_{t}\left(\theta_{1}, \theta_{2}, \cdots, \theta_{\nu-1}\right)$ if $l \leq t \leq 2 l-1$; when $l=\nu$, $\widehat{w}_{t}=\widehat{w}_{t}\left(\theta_{1}, \theta_{2}, \cdots, \theta_{\nu}\right)$ if $1 \leq t \leq \nu-1$ or $t=2 \nu-1$, and $\widehat{w}_{t}=\widehat{w}_{t}\left(\theta_{1}, \theta_{2}, \cdots, \theta_{\nu-1}\right)$ if $\nu \leq t<2 \nu-1$.

We now expand $\operatorname{det}\left(\widehat{\mathbf{W}}_{l}\right)$ by minors down its last column and write it into a polynomial in $\theta_{\nu}$. This yields

$$
\begin{aligned}
& (-1)^{\frac{1}{2} l(l+1)+1} \operatorname{det}\left(\widehat{\mathbf{W}}_{l}\right) \\
= & \widehat{w}_{2 l-1} \widehat{w}_{l-1}^{l-1}+\cdots \\
= & \begin{cases}\left(\sum_{s=1}^{l} r_{s l} \theta_{s}\right) \eta_{\nu}^{l-1} \theta_{\nu}^{l-1}+c_{l-2} \theta_{\nu}^{l-2}+\cdots+c_{1} \theta_{\nu}+c_{0} & \text { if } 2 \leq l<\nu, \\
r_{\nu \nu} \eta_{\nu}^{\nu-1} \theta_{\nu}^{\nu}+d_{\nu-1} \theta_{\nu}^{\nu-1}+\cdots+d_{1} \theta_{\nu}+d_{0} & \text { if } l=\nu\end{cases}
\end{aligned}
$$

where the coefficients $c_{0}, \cdots, c_{l-2}$ and $d_{0}, \cdots, d_{\nu-1}$ are polynomials in $\theta_{1}, \theta_{2}, \cdots, \theta_{\nu-1}$. Now (3.3) follows from the facts that $r_{l l} \neq 0, r_{\nu \nu} \neq 0, \eta_{\nu} \neq 0$ and $\theta_{1}, \theta_{2}, \cdots, \theta_{\nu}$ are independent random variables.

Note that $\nu$ is also the degree of the minimal polynomial of $\mathbf{A} \widehat{\mathbf{r}}_{0}$ with respect to $\mathbf{A}$ when $\mathbf{A}$ is nonsingular. With $\widehat{\mathbf{r}}_{0}$ replaced by $\mathbf{A} \widehat{\mathbf{r}}_{0}$ in (3.3), we then have

$$
\operatorname{Prob}\left(\operatorname{det}\left(\widehat{\mathbf{S}}_{l}\right)=0\right)=0
$$

for any $l$ with $1 \leq l \leq \nu$.

Now, (3.3) and (3.4) together imply that

$$
\begin{aligned}
& \operatorname{Prob}\left(\prod_{l=1}^{\nu} \operatorname{det}\left(\widehat{\mathbf{W}}_{l}\right) \operatorname{det}\left(\widehat{\mathbf{S}}_{l}\right)=0\right) \\
& \leq \sum_{l=1}^{\nu} \operatorname{Prob}\left(\operatorname{det}\left(\widehat{\mathbf{W}}_{l}\right)=0\right)+\sum_{l=1}^{\nu} \operatorname{Prob}\left(\operatorname{det}\left(\widehat{\mathbf{S}}_{l}\right)=0\right)=0
\end{aligned}
$$

and the lemma is proved.

The $\mathbf{A}$ in Lemma 3.1 is assumed to be nonsingular. For a general $\mathbf{A}$, we have

Theorem 3.1. Consider the case where $n=1, \widehat{\mathbf{r}}_{0} \in \mathcal{R}^{N}, \widehat{\mathbf{r}}_{0} \neq \mathbf{0}$ and $\mathbf{A} \in \mathcal{R}^{N \times N}$. If $\mathbf{q}_{1} \in$ $\mathcal{R}^{N}$ is a random vector with independent and identically distributed elements from $N(0,1)$, then $\operatorname{Prob}\left(\prod_{l=1}^{\nu} \operatorname{det}\left(\widehat{\mathbf{W}}_{l}\right) \operatorname{det}\left(\widehat{\mathbf{S}}_{l}\right)=0\right)=0$ if and only if $p_{\text {min }}\left(0 ; \mathbf{A}, \widehat{\mathbf{r}}_{0}\right) \neq 0$. 
Proof. If $p_{\min }\left(0 ; \mathbf{A}, \widehat{\mathbf{r}}_{0}\right)=0$, then $\mathbf{A}^{\nu} \widehat{\mathbf{r}}_{0}$ is a linear combination of $\mathbf{A} \widehat{\mathbf{r}}_{0}, \cdots, \mathbf{A}^{\nu-1} \widehat{\mathbf{r}}_{0}$ or $\mathbf{A} \widehat{\mathbf{r}}_{0}=\mathbf{0}$ in the case when $\nu=1$. Hence $\operatorname{det}\left(\widehat{\mathbf{S}}_{\nu}\right)=0$ no matter what $\mathbf{q}_{1}$ is and therefore $\operatorname{Prob}\left(\prod_{l=1}^{\nu} \operatorname{det}\left(\widehat{\mathbf{W}}_{l}\right) \operatorname{det}\left(\widehat{\mathbf{S}}_{l}\right)=0\right)=$ 1.

We now suppose $p_{\min }\left(0 ; \mathbf{A}, \widehat{\mathbf{r}}_{0}\right) \neq 0$. By the real version of the Schur's unitary triangularization theorem (see, for instance, [11]), A can be decomposed as

$$
\mathbf{A}=\mathbf{Q}^{T}\left[\begin{array}{cc}
\mathbf{B}_{11} & \mathbf{B}_{12} \\
\mathbf{0} & \mathbf{B}_{22}
\end{array}\right] \mathbf{Q} \equiv \mathbf{Q}^{T} \mathbf{B} \mathbf{Q}
$$

where $\mathbf{Q} \in \mathcal{R}^{N \times N}$ is orthogonal, $\mathbf{B}_{11} \in \mathcal{R}^{N_{1} \times N_{1}}$ nonsingular and $\mathbf{B}_{22} \in \mathcal{R}^{N_{2} \times N_{2}}$ strictly upper triangular (namely, an upper triangular matrix with its main diagonal elements zero). Let $\widetilde{\mathbf{r}}_{0}=$ $\mathbf{Q} \widehat{\mathbf{r}}_{0} \equiv\left[\widetilde{\mathbf{r}}_{01}^{T}, \widetilde{\mathbf{r}}_{02}^{T}\right]^{T}$ where $\widetilde{\mathbf{r}}_{01} \in \mathcal{R}^{N_{1}}$ and $\widetilde{\mathbf{r}}_{02} \in \mathcal{R}^{N_{2}}$. Then $p_{\min }\left(\mathbf{B} ; \mathbf{A}, \widehat{\mathbf{r}}_{0}\right) \widetilde{\mathbf{r}}_{0}=\mathbf{Q} p_{\min }\left(\mathbf{A} ; \mathbf{A}, \widehat{\mathbf{r}}_{0}\right) \widehat{\mathbf{r}}_{0}=$ 0. Note that

$$
\mathbf{B}^{k}=\left[\begin{array}{cc}
\mathbf{B}_{11}^{k} & * \\
\mathbf{0} & \mathbf{B}_{22}^{k}
\end{array}\right]
$$

for $k \in \mathcal{N}$, we have

$$
p_{\min }\left(\mathbf{B} ; \mathbf{A}, \widehat{\mathbf{r}}_{0}\right)=\left[\begin{array}{cc}
p_{\min }\left(\mathbf{B}_{11} ; \mathbf{A}, \widehat{\mathbf{r}}_{0}\right) & { }^{*} \\
\mathbf{0} & p_{\min }\left(\mathbf{B}_{22} ; \mathbf{A}, \widehat{\mathbf{r}}_{0}\right)
\end{array}\right] .
$$

Thus, $p_{\min }\left(\mathbf{B} ; \mathbf{A}, \widehat{\mathbf{r}}_{0}\right) \widetilde{\mathbf{r}}_{0}=\mathbf{0}$ implies that $p_{\min }\left(\mathbf{B}_{22} ; \mathbf{A}, \widehat{\mathbf{r}}_{0}\right) \widetilde{\mathbf{r}}_{02}=\mathbf{0}$. If we write $p_{\min }\left(\lambda ; \mathbf{A}, \widehat{\mathbf{r}}_{0}\right)=$ $\sum_{t=0}^{\nu} c_{t} \lambda^{t}$ with $c_{0} \neq 0$, then $\sum_{t=1}^{\nu} c_{t} \mathbf{B}_{22}^{t}$ is a strictly upper triangular matrix. Thus, $p_{\min }\left(\mathbf{B}_{22} ; \mathbf{A}, \widehat{\mathbf{r}}_{0}\right)=$ $\left(\sum_{t=1}^{\nu} c_{t} \mathbf{B}_{22}^{t}\right)+c_{0} \mathbf{I}$ is an upper triangular matrix whose main diagonal elements are $c_{0}$. So, $p_{\min }\left(\mathbf{B}_{22} ; \mathbf{A}, \widehat{\mathbf{r}}_{0}\right)$ is nonsingular and therefore $p_{\min }\left(\mathbf{B}_{22} ; \mathbf{A}, \widehat{\mathbf{r}}_{0}\right) \widetilde{\mathbf{r}}_{02}=\mathbf{0}$ yields $\widetilde{\mathbf{r}}_{02}=\mathbf{0}$. Since $\widetilde{\mathbf{r}}_{0} \neq \mathbf{0}$ due to $\widehat{\mathbf{r}}_{0} \neq \mathbf{0}$ by assumption, $\widetilde{\mathbf{r}}_{02} \neq \widetilde{\mathbf{r}}_{0}$. In other words, $N_{2}<N$ or $\mathbf{B}_{11}$ is not a null matrix.

Now that $\widetilde{\mathbf{r}}_{02}=\mathbf{0},(3.5)$ implies that

$$
\mathbf{B}^{k} \widetilde{\mathbf{r}}_{0}=\left[\begin{array}{c}
\mathbf{B}_{11}^{k} \widetilde{\mathbf{r}}_{01} \\
\mathbf{0}
\end{array}\right]
$$

for $k \in \mathcal{N}$. Therefore, $p(\mathbf{B}) \widetilde{\mathbf{r}}_{0}=\left[\left(p\left(\mathbf{B}_{11}\right) \widetilde{\mathbf{r}}_{01}\right)^{T}, \mathbf{0}^{T}\right]^{T}$ for any polynomial $p(\lambda)$. Thus, the minimal polynomial of $\widetilde{\mathbf{r}}_{0}$ with respect to $\mathbf{B}$ is equal to the minimal polynomial of $\widetilde{\mathbf{r}}_{01}$ with respect to $\mathbf{B}_{11}$. This implies that, $\nu$, the degree of the minimal polynomial of $\widehat{\mathbf{r}}_{0}$ with respect to $\mathbf{A}$, is also the degree of the minimal polynomial of $\widetilde{\mathbf{r}}_{01}$ with respect to $\mathbf{B}_{11}$.

We now set $\theta=\mathbf{Q} \mathbf{q}_{1} \equiv\left[\theta_{1}^{T}, \theta_{2}^{T}\right]^{T}$ where $\theta_{1} \in \mathcal{R}^{N_{1}}$ and $\theta_{2} \in \mathcal{R}^{N_{2}}$. Since $\mathbf{q}_{1}$ is random with iid elements from $N(0,1)$, so is $\theta$. By (3.6),

$$
\mathbf{q}_{1}^{T} \mathbf{A}^{k} \widehat{\mathbf{r}}_{0}=\theta^{T} \mathbf{B}^{k} \widetilde{\mathbf{r}}_{0}=\theta_{1}^{T} \mathbf{B}_{11}^{k} \widetilde{\mathbf{r}}_{01} \quad \text { and } \quad \mathbf{q}_{1}^{T} \widehat{\mathbf{r}}_{0}=\theta_{1}^{T} \widetilde{\mathbf{r}}_{01}
$$

where $k \in \mathcal{N}$. Thus

$$
\widehat{\mathbf{S}}_{\nu}\left(\mathbf{A}, \widehat{\mathbf{r}}_{0}, \mathbf{q}_{1}\right)=\widehat{\mathbf{S}}_{\nu}\left(\mathbf{B}_{11}, \widetilde{\mathbf{r}}_{01}, \theta_{1}\right) \text { and } \widehat{\mathbf{W}}_{\nu}\left(\mathbf{A}, \widehat{\mathbf{r}}_{0}, \mathbf{q}_{1}\right)=\widehat{\mathbf{W}}_{\nu}\left(\mathbf{B}_{11}, \widetilde{\mathbf{r}}_{01}, \theta_{1}\right) .
$$

Now, the desired probability follows from Lemma 3.1 because $\mathbf{B}_{11}$ is nonsingular, $\theta_{1}$ is iid $N(0,1)$ random and $\nu$ is the degree of the minimal polynomial of $\widetilde{\mathbf{r}}_{01}$ with respect to $\mathbf{B}_{11}$.

Extension of the theorem to the general case should be possible, namely, $n \geq 1, \mathbf{A} \in \mathcal{C}^{N \times N}, \widehat{\mathbf{r}}_{0} \in$ $\mathcal{C}^{N \times 1}$ and $\left[\mathbf{q}_{1}, \cdots, \mathbf{q}_{n}\right]$ is a Gaussian matrix. We remark that, when $\mathbf{A}$ is non-defective, the general case has been proved in the proof of Theorem 3 of [30. The proof was based on the observation that, if a polynomial $p\left(\lambda_{1}, \cdots, \lambda_{l}\right) \not \equiv 0$, then $\operatorname{Prob}\left(p\left(\lambda_{1}, \cdots, \lambda_{l}\right)=0\right)=0$ when $\lambda_{1}, \cdots, \lambda_{l}$ are randomly chosen.

Remark: $p_{\min }\left(0 ; \mathbf{A}, \widehat{\mathbf{r}}_{0}\right) \neq 0$ if and only if the affine space $\widehat{\mathbf{x}}_{0}+\operatorname{span}\left\{\mathbf{A}^{t} \widehat{\mathbf{r}}_{0} \mid t \in \mathcal{N}_{0}\right\}$ contains a solution to (3.1). 
The following corollary then follows from Proposition 3.1 and Theorem 3.1.

Corollary 3.2. In the case where $n=1$, (3.1) is a real system and $\mathbf{q}_{1} \in \mathcal{R}^{N}$ is a random vector with iid elements from $N(0,1)$, Algorithm 3.1 almost surely works without breakdown by zero division to find a solution from the affine space $\widehat{\mathbf{x}}_{0}+\operatorname{span}\left\{\mathbf{A}^{t} \widehat{\mathbf{r}}_{0} \mid t \in \mathcal{N}_{0}\right\}$ provided that $\widehat{\mathbf{x}}_{0} \in \mathcal{R}^{N}$ is chosen such that the affine space contains a solution to (3.1).

Remarks:

(i) The initial guess $\widehat{\mathbf{x}}_{0}$ in Corollary 3.2 is a user-provided vector. It may not be a random vector in some applications. For example, in cases where a sequence of similar linear systems is solved, the solution from the previous system may be used as the $\widehat{\mathbf{x}}_{0}$ for the new system.

(ii) If we pick $\widehat{\mathbf{x}}_{0} \in \mathcal{R}^{N}$ randomly and set $\mathbf{q}_{1}=\mathbf{b}-\mathbf{A} \widehat{\mathbf{x}}_{0}$, then Algorithm 3.1 with $n=1$, or equivalently in mathematics, the standard BiCG (see 96 ), almost surely solves (3.1) without breakdown by zero division for all, but a certain small class of, nonsingular $\mathbf{A} \in$ $\mathcal{R}^{N \times N}$. For details, see [13].

\section{A $\operatorname{ML}(n)$ BiCGStab Algorithm}

An algorithm for the ML $(n)$ BiCGStab method has been derived from ML $(n) \operatorname{BiCG}$ in 39 ] (Algorithm 2 without preconditioning and Algorithm 3 with preconditioning in [39]), but the derivation there is complicated and less inspiring. In this section, we re-derive the algorithm in a more systematic fashion with the help of index functions.

4.1. Notation and Definitions. Let $\phi_{k}(\lambda)$ be the polynomial of degree $k$ defined by (1.1). If expressed in terms of the power basis

$$
\phi_{k}(\lambda)=c_{k}^{(k)} \lambda^{k}+\cdots+c_{1}^{(k)} \lambda+c_{0}^{(k)},
$$

it is clear that $c_{k}^{(k)}=\rho_{1} \rho_{2} \cdots \rho_{k}$ and $c_{0}^{(k)}=1$. Thus,

$$
c_{k}^{(k)}=\rho_{k} c_{k-1}^{(k-1)} .
$$

In $\operatorname{ML}(n)$ BiCGStab, we construct the following vectors: for $k \in \mathcal{N}$,

$$
\begin{aligned}
& \mathbf{r}_{k}=\phi_{g_{n}(k)+1}(\mathbf{A}) \widehat{\mathbf{r}}_{k}, \quad \mathbf{u}_{k}=\phi_{g_{n}(k)}(\mathbf{A}) \widehat{\mathbf{r}}_{k}, \\
& \mathbf{g}_{k}=\phi_{g_{n}(k)+1}(\mathbf{A}) \widehat{\mathbf{g}}_{k}, \\
& \mathbf{w}_{k}=\mathbf{A g}_{k}=\rho_{g_{n}(k)+1} \mathbf{A} \phi_{g_{n}(k)}(\mathbf{A}) \widehat{\mathbf{g}}_{k},
\end{aligned}
$$

and for $k=0$, set

$$
\mathbf{r}_{0}=\widehat{\mathbf{r}}_{0} \quad \text { and } \quad \mathbf{g}_{0}=\widehat{\mathbf{g}}_{0} .
$$

The vectors $\mathbf{r}_{k}$ will be the residual vectors of the approximate solutions $\mathbf{x}_{k}$ computed in the following $\mathrm{ML}(n) \mathrm{BiCGStab}$ algorithm.

4.2. Algorithm Derivation. The derivation parallels the one of BiCGStab from BiCG. We first replace all the inner products $\mathbf{p}^{H} \widehat{\mathbf{r}}$ and $\mathbf{p}^{H} \mathbf{A} \widehat{\mathbf{g}}$ in $\mathrm{ML}(n)$ BiCG respectively by inner products of the forms $\mathbf{q}^{H} \phi(\mathbf{A}) \widehat{\mathbf{r}}$ and $\mathbf{q}^{H} \mathbf{A} \phi(\mathbf{A}) \widehat{\mathbf{g}}$, where $\phi$ is the polynomial (1.1). Corollary 3.1 guarantees that the inner products remain unchanged with such replacements. Then we compile recurrences for the new residuals $\mathbf{r}_{k}$ and the corresponding iterates. The overall derivation is best described and verified in stages, and depends on Proposition 2.1 and Corollary 3.1.

The derivation is complicated by the fact that the recurrences in the $k$ th iteration in $\operatorname{ML}(n) \operatorname{BiCG}$ involve $n$ terms which stretch from $k-n$ to $k-1$. Note that $k-n \leq g_{n}(k) n \leq k-1$. The degrees of the $\phi_{g_{n}(s)}$ and $\phi_{g_{n}(s)+1}$ in (4.3) are increased at $g_{n}(k) n+1$ as $s$ runs from $k-n$ to $k-1$ (see, 
for example, Table 2.1). Therefore, our first task in the derivation is to split up in $\mathrm{ML}(n) \operatorname{BiCG}$ the loops and the sums of length $n$ into two parts, one from $k-n$ to $g_{n}(k) n-1$ and the other from $g_{n}(k) n+1$ to $k-1$. The following Derivation Stage (DS) \#1 is computationally equivalent to Algorithm 3.1 (forgetting Lines 1, 2, 5 and 11).

\section{Derivation Stage \#1.}

1. For $k=1,2, \cdots$, until convergence:

2. If $r_{n}(k)=1$

3. $\quad \alpha_{k}=\mathbf{p}_{k}^{H} \widehat{\mathbf{r}}_{k-1} / \mathbf{p}_{k}^{H} \mathbf{A} \widehat{\mathbf{g}}_{k-1} ;$

4. $\quad \widehat{\mathbf{r}}_{k}=\widehat{\mathbf{r}}_{k-1}-\alpha_{k} \mathbf{A} \widehat{\mathbf{g}}_{k-1}$;

5. $\quad$ Else

6. $\quad \alpha_{k}=\mathbf{p}_{k}^{H} \widehat{\mathbf{r}}_{k-1} / \mathbf{p}_{k}^{H} \mathbf{A} \widehat{\mathbf{g}}_{k-1} ;$

7. $\quad \widehat{\mathbf{r}}_{k}=\widehat{\mathbf{r}}_{k-1}-\alpha_{k} \mathbf{A} \widehat{\mathbf{g}}_{k-1}$;

8. $\quad$ End

9. If $r_{n}(k)<n$

10. $\quad$ For $s=\max (k-n, 0), \cdots, g_{n}(k) n-1$

11. $\beta_{s}^{(k)}=-\mathbf{p}_{s+1}^{H} \mathbf{A}\left(\widehat{\mathbf{r}}_{k}+\sum_{t=\max (k-n, 0)}^{s-1} \beta_{t}^{(k)} \widehat{\mathbf{g}}_{t}\right) / \mathbf{p}_{s+1}^{H} \mathbf{A} \widehat{\mathbf{g}}_{s} ;$

12. $\quad$ End

13. $\quad \beta_{g_{n}(k) n}^{(k)}=-\mathbf{p}_{g_{n}(k) n+1}^{H} \mathbf{A}\left(\widehat{\mathbf{r}}_{k}+\sum_{t=\max (k-n, 0)}^{g_{n}(k) n-1} \beta_{t}^{(k)} \widehat{\mathbf{g}}_{t}\right) / \mathbf{p}_{g_{n}(k) n+1}^{H} \mathbf{A} \widehat{\mathbf{g}}_{g_{n}(k) n} ;$

14. $\quad$ For $s=g_{n}(k) n+1, \cdots, k-1$

15. $\beta_{s}^{(k)}=-\mathbf{p}_{s+1}^{H} \mathbf{A}\left(\widehat{\mathbf{r}}_{k}+\sum_{t=\max (k-n, 0)}^{g_{n}(k) n} \beta_{t}^{(k)} \widehat{\mathbf{g}}_{t}+\sum_{t=g_{n}(k) n+1}^{s-1} \beta_{t}^{(k)} \widehat{\mathbf{g}}_{t}\right) / \mathbf{p}_{s+1}^{H} \mathbf{A} \widehat{\mathbf{g}}_{s} ;$

16. $\quad$ End

17. $\quad \widehat{\mathbf{g}}_{k}=\widehat{\mathbf{r}}_{k}+\sum_{s=\max (k-n, 0)}^{g_{n}(k) n} \beta_{s}^{(k)} \widehat{\mathbf{g}}_{s}+\sum_{s=g_{n}(k) n+1}^{k-1} \beta_{s}^{(k)} \widehat{\mathbf{g}}_{s} ;$

18. Else

19. $\quad \beta_{g_{n}(k) n}^{(k)}=-\mathbf{p}_{g_{n}(k) n+1}^{H} \mathbf{A} \widehat{\mathbf{r}}_{k} / \mathbf{p}_{g_{n}(k) n+1}^{H} \mathbf{A} \widehat{\mathbf{g}}_{g_{n}(k) n} ;$

20. $\quad$ For $s=g_{n}(k) n+1, \cdots, k-1$

21. $\quad \beta_{s}^{(k)}=-\mathbf{p}_{s+1}^{H} \mathbf{A}\left(\widehat{\mathbf{r}}_{k}+\beta_{g_{n}(k) n}^{(k)} \widehat{\mathbf{g}}_{g_{n}(k) n}+\sum_{t=g_{n}(k) n+1}^{s-1} \beta_{t}^{(k)} \widehat{\mathbf{g}}_{t}\right) / \mathbf{p}_{s+1}^{H} \mathbf{A} \widehat{\mathbf{g}}_{s} ;$

22. $\quad$ End

23. $\quad \widehat{\mathbf{g}}_{k}=\widehat{\mathbf{r}}_{k}+\beta_{g_{n}(k) n}^{(k)} \widehat{\mathbf{g}}_{g_{n}(k) n}+\sum_{s=g_{n}(k) n+1}^{k-1} \beta_{s}^{(k)} \widehat{\mathbf{g}}_{s} ;$

24. End

25. End

We have adopted the conventions: empty loops are skipped and empty sums are zero. These conventions will also be applied in the sequel.

In the next stage of the derivation, we replace inner products $\mathbf{p}^{H} \widehat{\mathbf{r}}$ and $\mathbf{p}^{H} \mathbf{A} \widehat{\mathbf{g}}$ by inner products of the forms $\mathbf{q}^{H} \phi(\mathbf{A}) \widehat{\mathbf{r}}$ and $\mathbf{q}^{H} \mathbf{A} \phi(\mathbf{A}) \widehat{\mathbf{g}}$ respectively. That is, the factor $\left(\mathbf{A}^{H}\right)^{g_{n}(k)}$ that is hidden in the left basis vector $\mathbf{p}_{k}$ is moved to the right-hand side space and replaced by the factor $\phi_{g_{n}(k)}(\mathbf{A})$. Formally, by Corollary 3.1 together with (3.2), (4.2) and Proposition 2.1(a), DS\#1 can be further transformed into the version below. Explanations are given after listing.

\section{Derivation Stage \#2.}

1. For $k=1,2, \cdots$, until convergence:

2. If $r_{n}(k)=1$

3. $\quad \alpha_{k}=\mathbf{q}_{r_{n}(k)}^{H} \phi_{g_{n}(k)}(\mathbf{A}) \widehat{\mathbf{r}}_{k-1} / \mathbf{q}_{r_{n}(k)}^{H} \mathbf{A} \phi_{g_{n}(k)}(\mathbf{A}) \widehat{\mathbf{g}}_{k-1} ;$

4. $\quad \phi_{g_{n}(k)}(\mathbf{A}) \widehat{\mathbf{r}}_{k}=\phi_{g_{n}(k)}(\mathbf{A}) \widehat{\mathbf{r}}_{k-1}-\alpha_{k} \mathbf{A} \phi_{g_{n}(k)}(\mathbf{A}) \widehat{\mathbf{g}}_{k-1} ;$ 
5. $\quad \phi_{g_{n}(k)+1}(\mathbf{A}) \widehat{\mathbf{r}}_{k}=\left(\rho_{g_{n}(k)+1} \mathbf{A}+\mathbf{I}\right) \phi_{g_{n}(k)}(\mathbf{A}) \widehat{\mathbf{r}}_{k} ;$

6. Else

7. $\quad \alpha_{k}=\mathbf{q}_{r_{n}(k)}^{H} \phi_{g_{n}(k)}(\mathbf{A}) \widehat{\mathbf{r}}_{k-1} / \mathbf{q}_{r_{n}(k)}^{H} \mathbf{A} \phi_{g_{n}(k)}(\mathbf{A}) \widehat{\mathbf{g}}_{k-1} ;$

8. $\quad \phi_{g_{n}(k)}(\mathbf{A}) \widehat{\mathbf{r}}_{k}=\phi_{g_{n}(k)}(\mathbf{A}) \widehat{\mathbf{r}}_{k-1}-\alpha_{k} \mathbf{A} \phi_{g_{n}(k)}(\mathbf{A}) \widehat{\mathbf{g}}_{k-1}$;

9. $\quad \phi_{g_{n}(k)+1}(\mathbf{A}) \widehat{\mathbf{r}}_{k}=\phi_{g_{n}(k)+1}(\mathbf{A}) \widehat{\mathbf{r}}_{k-1}-\alpha_{k} \mathbf{A} \phi_{g_{n}(k)+1}(\mathbf{A}) \widehat{\mathbf{g}}_{k-1}$;

10. End

11. If $r_{n}(k)<n$

12. $\quad$ For $s=\max (k-n, 0), \cdots, g_{n}(k) n-1$

13. $\quad \beta_{s}^{(k)}=-\mathbf{q}_{r_{n}(s+1)}^{H}\left(\phi_{g_{n}(s+1)+1}(\mathbf{A}) \widehat{\mathbf{r}}_{k}+\right.$ $\left.\sum_{t=\max (k-n, 0)}^{s-1} \beta_{t}^{(k)} \rho_{g_{n}(s+1)+1} \mathbf{A} \phi_{g_{n}(s+1)}(\mathbf{A}) \widehat{\mathbf{g}}_{t}\right) / \rho_{g_{n}(s+1)+1} \mathbf{q}_{r_{n}(s+1)}^{H} \mathbf{A} \phi_{g_{n}(s+1)}(\mathbf{A}) \widehat{\mathbf{g}}_{s} ;$

14. $\quad$ End

15. $\quad \beta_{g_{n}(k) n}^{(k)}=-\mathbf{q}_{1}^{H}\left(\phi_{g_{n}(k)+1}(\mathbf{A}) \widehat{\mathbf{r}}_{k}+\right.$ $\left.\sum_{t=\max (k-n, 0)}^{g_{n}(k) n-1} \beta_{t}^{(k)} \rho_{g_{n}(k)+1} \mathbf{A} \phi_{g_{n}(k)}(\mathbf{A}) \widehat{\mathbf{g}}_{t}\right) / \rho_{g_{n}(k)+1} \mathbf{q}_{1}^{H} \mathbf{A} \phi_{g_{n}(k)}(\mathbf{A}) \widehat{\mathbf{g}}_{g_{n}(k) n}$

16. $\quad$ For $s=g_{n}(k) n+1, \cdots, k-1$

17. $\quad \beta_{s}^{(k)}=-\mathbf{q}_{r_{n}(s+1)}^{H}\left(\phi_{g_{n}(s+1)+1}(\mathbf{A}) \widehat{\mathbf{r}}_{k}+\sum_{t=\max (k-n, 0)}^{g_{n}(k) n} \beta_{t}^{(k)} \rho_{g_{n}(s+1)+1} \mathbf{A} \phi_{g_{n}(s+1)}(\mathbf{A}) \widehat{\mathbf{g}}_{t}+\right.$ $\left.\sum_{t=g_{n}(k) n+1}^{s-1} \beta_{t}^{(k)} \rho_{g_{n}(s+1)+1} \mathbf{A} \phi_{g_{n}(s+1)}(\mathbf{A}) \widehat{\mathbf{g}}_{t}\right) / \rho_{g_{n}(s+1)+1} \mathbf{q}_{r_{n}(s+1)}^{H} \mathbf{A} \phi_{g_{n}(s+1)}(\mathbf{A}) \widehat{\mathbf{g}}_{s} ;$

18. $\quad$ End

19. $\quad \rho_{g_{n}(k)+1} \mathbf{A} \phi_{g_{n}(k)}(\mathbf{A}) \widehat{\mathbf{g}}_{k}=\rho_{g_{n}(k)+1} \mathbf{A} \phi_{g_{n}(k)}(\mathbf{A}) \widehat{\mathbf{r}}_{k}+$ $\sum_{s=\max (k-n, 0)}^{g_{n}(k) n} \beta_{s}^{(k)} \rho_{g_{n}(k)+1} \mathbf{A} \phi_{g_{n}(k)}(\mathbf{A}) \widehat{\mathbf{g}}_{s}+$
$\sum_{s=g_{n}(k) n+1}^{k-1} \beta_{s}^{(k)} \rho_{g_{n}(k)+1} \mathbf{A} \phi_{g_{n}(k)}(\mathbf{A}) \widehat{\mathbf{g}}_{s} ;$

20. $\quad \phi_{g_{n}(k)+1}(\mathbf{A}) \widehat{\mathbf{g}}_{k}=\phi_{g_{n}(k)+1}(\mathbf{A}) \widehat{\mathbf{r}}_{k}+\sum_{s=\max (k-n, 0)}^{g_{n}(k) n} \beta_{s}^{(k)} \phi_{g_{n}(k)+1}(\mathbf{A}) \widehat{\mathbf{g}}_{s}+$ $\sum_{s=g_{n}(k) n+1}^{k-1} \beta_{s}^{(k)} \phi_{g_{n}(k)+1}(\mathbf{A}) \widehat{\mathbf{g}}_{s}$;

21. Else

22. $\quad \beta_{g_{n}(k) n}^{(k)}=-\mathbf{q}_{1}^{H} \phi_{g_{n}(k)+1}(\mathbf{A}) \widehat{\mathbf{r}}_{k} / \rho_{g_{n}(k)+1} \mathbf{q}_{1}^{H} \mathbf{A} \phi_{g_{n}(k)}(\mathbf{A}) \widehat{\mathbf{g}}_{g_{n}(k) n} ;$

23. $\quad$ For $s=g_{n}(k) n+1, \cdots, k-1$

24. $\quad \beta_{s}^{(k)}=-\mathbf{q}_{r_{n}(s+1)}^{H}\left(\phi_{g_{n}(s+1)+1}(\mathbf{A}) \widehat{\mathbf{r}}_{k}+\beta_{g_{n}(k) n}^{(k)} \rho_{g_{n}(s+1)+1} \mathbf{A} \phi_{g_{n}(s+1)}(\mathbf{A}) \widehat{\mathbf{g}}_{g_{n}(k) n}+\right.$ $\left.\sum_{t=g_{n}(k) n+1}^{s-1} \beta_{t}^{(k)} \rho_{g_{n}(s+1)+1} \mathbf{A} \phi_{g_{n}(s+1)}(\mathbf{A}) \widehat{\mathbf{g}}_{t}\right) / \rho_{g_{n}(s+1)+1} \mathbf{q}_{r_{n}(s+1)}^{H} \mathbf{A} \phi_{g_{n}(s+1)}(\mathbf{A}) \widehat{\mathbf{g}}_{s} ;$

25. $\quad$ End

26. $\quad \phi_{g_{n}(k)+1}(\mathbf{A}) \widehat{\mathbf{g}}_{k}=\phi_{g_{n}(k)+1}(\mathbf{A}) \widehat{\mathbf{r}}_{k}+\beta_{g_{n}(k) n}^{(k)} \phi_{g_{n}(k)+1}(\mathbf{A}) \widehat{\mathbf{g}}_{g_{n}(k) n}+$ $\sum_{s=g_{n}(k) n+1}^{k-1} \beta_{s}^{(k)} \phi_{g_{n}(k)+1}(\mathbf{A}) \widehat{\mathbf{g}}_{s}$;

27. End

28. End

Lines 4, 8, 9, 19, 20 and 26, DS\#2, were obtained from Lines 4, 7, 17 and 23, DS\#1, through a multiplication by $\phi_{g_{n}(k)}(\mathbf{A}), \phi_{g_{n}(k)+1}(\mathbf{A})$ and $\rho_{g_{n}(k)+1} \mathbf{A} \phi_{g_{n}(k)}(\mathbf{A})$ respectively. Line 5, DS\#2, is a direct result of the definition (1.1) of $\phi$. These lines are prepared for the updates of the vectors defined in (4.3).

To help understand how DS\#1 is turned into DS\#2, let us demonstrate (i) the transformation of Line 3, DS\#1, into Line 3, DS\#2 and (ii) the transformation of the term $\mathbf{p}_{g_{n}(k) n+1}^{H} \mathbf{A} \widehat{\mathbf{r}}_{k}$ on Line 13, DS\#1, into the term $\mathbf{q}_{1}^{H} \phi_{g_{n}(k)+1}(\mathbf{A}) \widehat{\mathbf{r}}_{k}$ on Line 15 , DS\#2, as follows. 
(i) By Corollary 3.1 ,

$$
\alpha_{k}=\frac{\mathbf{p}_{k}^{H} \widehat{\mathbf{r}}_{k-1}}{\mathbf{p}_{k}^{H} \mathbf{A} \widehat{\mathbf{g}}_{k-1}}=\frac{\frac{1}{c_{g_{n}(k)}^{\left(g_{n}(k)\right.}} \mathbf{q}_{r_{n}(k)}^{H} \phi_{g_{n}(k)}(\mathbf{A}) \widehat{\mathbf{r}}_{k-1}}{\frac{1}{c_{g_{n}(k)}^{\left(g_{n}(k)\right.}} \mathbf{q}_{r_{n}(k)}^{H} \mathbf{A} \phi_{g_{n}(k)}(\mathbf{A}) \widehat{\mathbf{g}}_{k-1}}=\frac{\mathbf{q}_{r_{n}(k)}^{H} \phi_{g_{n}(k)}(\mathbf{A}) \widehat{\mathbf{r}}_{k-1}}{\mathbf{q}_{r_{n}(k)}^{H} \mathbf{A} \phi_{g_{n}(k)}(\mathbf{A}) \widehat{\mathbf{g}}_{k-1}}
$$

where $c_{g_{n}(k)}^{\left(g_{n}(k)\right)}$ is the leading coefficient of $\phi_{g_{n}(k)}(\lambda)$ (see (4.1)).

(ii) By (3.2) and Proposition 2.1(a), we have

$$
\begin{aligned}
\mathbf{A}^{H} \mathbf{p}_{g_{n}(k) n+1} & =\left(\mathbf{A}^{H}\right)^{g_{n}\left(g_{n}(k) n+1\right)+1} \mathbf{q}_{r_{n}\left(g_{n}(k) n+1\right)} \\
& =\left(\mathbf{A}^{H}\right)^{g_{n}\left(\left(g_{n}(k)+1\right) n+1\right)} \mathbf{q}_{r_{n}\left(\left(g_{n}(k)+1\right) n+1\right)} \\
& =\mathbf{p}_{\left(g_{n}(k)+1\right) n+1} .
\end{aligned}
$$

Hence $\mathbf{p}_{g_{n}(k) n+1}^{H} \mathbf{A} \widehat{\mathbf{r}}_{k}=\mathbf{p}_{\left(g_{n}(k)+1\right) n+1}^{H} \widehat{\mathbf{r}}_{k}$. Since $\left(g_{n}(k)+1\right) n+1 \leq k+n$, an application of Corollary 3.1 to $\mathbf{p}_{\left(g_{n}(k)+1\right) n+1}^{H} \widehat{\mathbf{r}}_{k}$ thus yields

$$
\begin{aligned}
\mathbf{p}_{g_{n}(k) n+1}^{H} \mathbf{A} \widehat{\mathbf{r}}_{k} & =\frac{1}{c_{\left.g_{n}\left(\left(g_{n}(k)+1\right) n+1\right)\right)}^{\left(g_{n}\left(g_{n}(k)+1\right) n+1\right)}} \mathbf{q}_{r_{n}\left(\left(g_{n}(k)+1\right) n+1\right)}^{H} \phi_{g_{n}\left(\left(g_{n}(k)+1\right) n+1\right)}(\mathbf{A}) \widehat{\mathbf{r}}_{k} \\
& =\frac{1}{c_{g_{n}(k)+1}^{\left(g_{n}(k)+1\right)}} \mathbf{q}_{1}^{H} \phi_{g_{n}(k)+1}(\mathbf{A}) \widehat{\mathbf{r}}_{k} .
\end{aligned}
$$

The second equation above follows from (2.2). The coefficient $1 / c_{g_{n}(k)+1}^{\left(g_{n}(k)+1\right)}$ is missed from Line 15, DS\#2, because it was canceled out by the coefficient from the denominator.

Our goal is to establish updating relations for the quantities introduced in (4.3). To this end, we further transform DS\#2 into the following version. This time, we work on the index function $g_{n}$ with the aid of Proposition 2.1 so that the definitions in (4.3) can be applied. Again, further explanations are given after the listing.

\section{Derivation Stage \#3.}

1. For $k=1,2, \cdots$, until convergence:

2. If $r_{n}(k)=1$

3. $\quad \alpha_{k}=\mathbf{q}_{r_{n}(k)}^{H} \phi_{g_{n}(k-1)+1}(\mathbf{A}) \widehat{\mathbf{r}}_{k-1} / \mathbf{q}_{r_{n}(k)}^{H} \mathbf{A} \phi_{g_{n}(k-1)+1}(\mathbf{A}) \widehat{\mathbf{g}}_{k-1} ;$

4. $\quad \phi_{g_{n}(k)}(\mathbf{A}) \widehat{\mathbf{r}}_{k}=\phi_{g_{n}(k-1)+1}(\mathbf{A}) \widehat{\mathbf{r}}_{k-1}-\alpha_{k} \mathbf{A} \phi_{g_{n}(k-1)+1}(\mathbf{A}) \widehat{\mathbf{g}}_{k-1}$;

5. $\quad \phi_{g_{n}(k)+1}(\mathbf{A}) \widehat{\mathbf{r}}_{k}=\left(\rho_{g_{n}(k)+1} \mathbf{A}+\mathbf{I}\right) \phi_{g_{n}(k)}(\mathbf{A}) \widehat{\mathbf{r}}_{k}$

6. Else

7. $\quad \alpha_{k}=\mathbf{q}_{r_{n}(k)}^{H} \phi_{g_{n}(k-1)}(\mathbf{A}) \widehat{\mathbf{r}}_{k-1} / \mathbf{q}_{r_{n}(k)}^{H} \mathbf{A} \phi_{g_{n}(k-1)}(\mathbf{A}) \widehat{\mathbf{g}}_{k-1}$

8. $\quad \phi_{g_{n}(k)}(\mathbf{A}) \widehat{\mathbf{r}}_{k}=\phi_{g_{n}(k-1)}(\mathbf{A}) \widehat{\mathbf{r}}_{k-1}-\alpha_{k} \mathbf{A} \phi_{g_{n}(k-1)}(\mathbf{A}) \widehat{\mathbf{g}}_{k-1}$;

9. $\quad \phi_{g_{n}(k)+1}(\mathbf{A}) \widehat{\mathbf{r}}_{k}=\phi_{g_{n}(k-1)+1}(\mathbf{A}) \widehat{\mathbf{r}}_{k-1}-\alpha_{k} \mathbf{A} \phi_{g_{n}(k-1)+1}(\mathbf{A}) \widehat{\mathbf{g}}_{k-1}$;

10. End

11. If $r_{n}(k)<n$

12. $\quad$ For $s=\max (k-n, 0), \cdots, g_{n}(k) n-1$

13. $\quad \beta_{s}^{(k)}=-\mathbf{q}_{r_{n}(s+1)}^{H}\left(\phi_{g_{n}(k)}(\mathbf{A}) \widehat{\mathbf{r}}_{k}+\right.$

$$
\left.\sum_{t=\max (k-n, 0)}^{s-1} \beta_{t}^{(k)} \rho_{g_{n}(t)+1} \mathbf{A} \phi_{g_{n}(t)}(\mathbf{A}) \widehat{\mathbf{g}}_{t}\right) / \rho_{g_{n}(s)+1} \mathbf{q}_{r_{n}(s+1)}^{H} \mathbf{A} \phi_{g_{n}(s)}(\mathbf{A}) \widehat{\mathbf{g}}_{s} ;
$$

14. $\quad$ End

15. $\quad \beta_{g_{n}(k) n}^{(k)}=-\mathbf{q}_{1}^{H}\left(\phi_{g_{n}(k)+1}(\mathbf{A}) \widehat{\mathbf{r}}_{k}+\right.$

$$
\left.\sum_{t=\max (k-n, 0)}^{g_{n}(k) n-1} \beta_{t}^{(k)} \rho_{g_{n}(k)+1} \mathbf{A} \phi_{g_{n}(t)+1}(\mathbf{A}) \widehat{\mathbf{g}}_{t}\right) / \rho_{g_{n}(k)+1} \mathbf{q}_{1}^{H} \mathbf{A} \phi_{g_{n}\left(g_{n}(k) n\right)+1}(\mathbf{A}) \widehat{\mathbf{g}}_{g_{n}(k) n} ;
$$

16. $\quad$ For $s=g_{n}(k) n+1, \cdots, k-1$

17. $\quad \beta_{s}^{(k)}=-\mathbf{q}_{r_{n}(s+1)}^{H}\left(\phi_{g_{n}(k)+1}(\mathbf{A}) \widehat{\mathbf{r}}_{k}+\sum_{t=\max (k-n, 0)}^{g_{n}(k) n} \beta_{t}^{(k)} \rho_{g_{n}(k)+1} \mathbf{A} \phi_{g_{n}(t)+1}(\mathbf{A}) \widehat{\mathbf{g}}_{t}+\right.$ 
18. $\quad$ End

$$
\left.\sum_{t=g_{n}(k) n+1}^{s-1} \beta_{t}^{(k)} \rho_{g_{n}(t)+1} \mathbf{A} \phi_{g_{n}(t)}(\mathbf{A}) \widehat{\mathbf{g}}_{t}\right) / \rho_{g_{n}(s)+1} \mathbf{q}_{r_{n}(s+1)}^{H} \mathbf{A} \phi_{g_{n}(s)}(\mathbf{A}) \widehat{\mathbf{g}}_{s}
$$

19. $\quad \rho_{g_{n}(k)+1} \mathbf{A} \phi_{g_{n}(k)}(\mathbf{A}) \widehat{\mathbf{g}}_{k}=\rho_{g_{n}(k)+1} \mathbf{A} \phi_{g_{n}(k)}(\mathbf{A}) \widehat{\mathbf{r}}_{k}+$

$$
\begin{aligned}
& \sum_{s=\max (k-n, 0)}^{g_{n}(k) n} \beta_{s}^{(k)} \rho_{g_{n}(k)+1} \mathbf{A} \phi_{g_{n}(s)+1}(\mathbf{A}) \widehat{\mathbf{g}}_{s}+ \\
& \sum_{s=g_{n}(k) n+1}^{k-1} \beta_{s}^{(k)} \rho_{g_{n}(s)+1} \mathbf{A} \phi_{g_{n}(s)}(\mathbf{A}) \widehat{\mathbf{g}}_{s}
\end{aligned}
$$

20.

$$
\begin{aligned}
\phi_{g_{n}(k)+1}(\mathbf{A}) \widehat{\mathbf{g}}_{k}= & \phi_{g_{n}(k)+1}(\mathbf{A}) \widehat{\mathbf{r}}_{k}+\sum_{s=\max (k-n, 0)}^{g_{n}(k) n} \beta_{s}^{(k)}\left(\rho_{g_{n}(k)+1} \mathbf{A}+\mathbf{I}\right) \phi_{g_{n}(s)+1}(\mathbf{A}) \widehat{\mathbf{g}}_{s}+ \\
& \sum_{s=g_{n}(k) n+1}^{k-1} \beta_{s}^{(k)} \phi_{g_{n}(s)+1}(\mathbf{A}) \widehat{\mathbf{g}}_{s} ;
\end{aligned}
$$

21. $\quad$ Else

$$
\beta_{g_{n}(k) n}^{(k)}=-\mathbf{q}_{1}^{H} \phi_{g_{n}(k)+1}(\mathbf{A}) \widehat{\mathbf{r}}_{k} / \rho_{g_{n}(k)+1} \mathbf{q}_{1}^{H} \mathbf{A} \phi_{g_{n}\left(g_{n}(k) n\right)+1}(\mathbf{A}) \widehat{\mathbf{g}}_{g_{n}(k) n} ;
$$

23. $\quad$ For $s=g_{n}(k) n+1, \cdots, k-1$

24 .

25.

$$
\begin{gathered}
\beta_{s}^{(k)}=-\mathbf{q}_{r_{n}(s+1)}^{H}\left(\phi_{g_{n}(k)+1}(\mathbf{A}) \widehat{\mathbf{r}}_{k}+\beta_{g_{n}(k) n}^{(k)} \rho_{g_{n}(k)+1} \mathbf{A} \phi_{g_{n}\left(g_{n}(k) n\right)+1}(\mathbf{A}) \widehat{\mathbf{g}}_{g_{n}(k) n}+\right. \\
\left.\sum_{t=g_{n}(k) n+1}^{s-1} \beta_{t}^{(k)} \rho_{g_{n}(t)+1} \mathbf{A} \phi_{g_{n}(t)}(\mathbf{A}) \widehat{\mathbf{g}}_{t}\right) / \rho_{g_{n}(s)+1} \mathbf{q}_{r_{n}(s+1)}^{H} \mathbf{A} \phi_{g_{n}(s)}(\mathbf{A}) \widehat{\mathbf{g}}_{s}
\end{gathered}
$$

26. $\quad \phi_{g_{n}(k)+1}(\mathbf{A}) \widehat{\mathbf{g}}_{k}=\phi_{g_{n}(k)+1}(\mathbf{A}) \widehat{\mathbf{r}}_{k}+\beta_{g_{n}(k) n}^{(k)}\left(\rho_{g_{n}(k)+1} \mathbf{A}+\mathbf{I}\right) \phi_{g_{n}\left(g_{n}(k) n\right)+1}(\mathbf{A}) \widehat{\mathbf{g}}_{g_{n}(k) n}+$

$$
\sum_{s=g_{n}(k) n+1}^{k-1} \beta_{s}^{(k)} \phi_{g_{n}(s)+1}(\mathbf{A}) \widehat{\mathbf{g}}_{s} \text {; }
$$

27. End

28. End

As an example, let us show how the $g_{n}(s+1)$ inside the sum $\sum_{t=\max (k-n, 0)}^{s-1} \cdots$ on Line 13, DS\#2, was written as the $g_{n}(t)$ on Line 13 , DS\#3.

If $g_{n}(k)=0$, Line 13 of DS\#2 is not implemented because of the conventions immediately following DS\#1. So, we assume that $g_{n}(k)>0$. Since

$$
\max (k-n, 0) \leq s, t \leq g_{n}(k) n-1,
$$

we have

$$
g_{n}(s+1)=g_{n}(k+1)-1=g_{n}(t+1)
$$

by Proposition 2.1(b). Now that $g_{n}(k)>0, \max (k-n, 0)=k-n$ and hence

$$
k-n \leq t \leq g_{n}(k) n-1 .
$$

Let $k=j n+i$ as in (2.1). Then (4.5) is

$$
(j-1) n+i \leq t \leq(j-1) n+n-1
$$

which implies that $r_{n}(t)<n$. Now, Proposition 2.1(d) yields $g_{n}(t+1)=g_{n}(t)$ and therefore we have $g_{n}(s+1)=g_{n}(t)$.

Now we are ready to use the vectors defined in (4.3) and (4.4). Substituting these vectors into DS\#3 leads to the following stage.

\section{Derivation Stage \#4.}

1. For $k=1,2, \cdots$, until convergence:

2. If $r_{n}(k)=1$

3. $\quad \alpha_{k}=\mathbf{q}_{r_{n}(k)}^{H} \mathbf{r}_{k-1} / \mathbf{q}_{r_{n}(k)}^{H} \mathbf{A g}_{k-1} ;$

4. $\quad \mathbf{u}_{k}=\mathbf{r}_{k-1}-\alpha_{k} \mathbf{A g}_{k-1}$;

5. $\quad \mathbf{r}_{k}=\rho_{g_{n}(k)+1} \mathbf{A} \mathbf{u}_{k}+\mathbf{u}_{k}$;

6. Else

7. $\alpha_{k}=\rho_{g_{n}(k-1)+1} \mathbf{q}_{r_{n}(k)}^{H} \mathbf{u}_{k-1} / \mathbf{q}_{r_{n}(k)}^{H} \mathbf{d}_{k-1} ;$ 
8. $\quad \mathbf{u}_{k}=\mathbf{u}_{k-1}-\left(\alpha_{k} / \rho_{g_{n}(k-1)+1}\right) \mathbf{d}_{k-1} ;$

9. $\quad \mathbf{r}_{k}=\mathbf{r}_{k-1}-\alpha_{k} \mathbf{A} \mathbf{g}_{k-1}$

10. End

11. If $r_{n}(k)<n$

12. For $s=\max (k-n, 0), \cdots, g_{n}(k) n-1$

13.

14. $\quad$ End

$$
\beta_{s}^{(k)}=-\mathbf{q}_{r_{n}(s+1)}^{H}\left(\mathbf{u}_{k}+\sum_{t=\max (k-n, 0)}^{s-1} \beta_{t}^{(k)} \mathbf{d}_{t}\right) / \mathbf{q}_{r_{n}(s+1)}^{H} \mathbf{d}_{s} ;
$$

15. $\quad \beta_{g_{n}(k) n}^{(k)}=-\mathbf{q}_{1}^{H}\left(\mathbf{r}_{k}+\rho_{g_{n}(k)+1} \sum_{t=\max (k-n, 0)}^{g_{n}(k) n-1} \beta_{t}^{(k)} \mathbf{A g}_{t}\right) / \rho_{g_{n}(k)+1} \mathbf{q}_{1}^{H} \mathbf{A g}_{g_{n}(k) n}$;

16. $\quad$ For $s=g_{n}(k) n+1, \cdots, k-1$

17. $\quad \beta_{s}^{(k)}=-\mathbf{q}_{r_{n}(s+1)}^{H}\left(\mathbf{r}_{k}+\rho_{g_{n}(k)+1} \sum_{t=\max (k-n, 0)}^{g_{n}(k) n} \beta_{t}^{(k)} \mathbf{A g}_{t}+\right.$

$$
\left.\sum_{t=g_{n}(k) n+1}^{s-1} \beta_{t}^{(k)} \mathbf{d}_{t}\right) / \mathbf{q}_{r_{n}(s+1)}^{H} \mathbf{d}_{s} \text {; }
$$

18. $\quad$ End

19. $\quad \mathbf{d}_{k}=\mathbf{r}_{k}-\mathbf{u}_{k}+\rho_{g_{n}(k)+1} \sum_{s=\max (k-n, 0)}^{g_{n}(k) n} \beta_{s}^{(k)} \mathbf{A g}_{s}+\sum_{s=g_{n}(k) n+1}^{k-1} \beta_{s}^{(k)} \mathbf{d}_{s} ;$

20. $\quad \mathbf{g}_{k}=\mathbf{r}_{k}+\sum_{s=\max (k-n, 0)}^{g_{n}(k) n} \beta_{s}^{(k)}\left(\rho_{g_{n}(k)+1} \mathbf{A}+\mathbf{I}\right) \mathbf{g}_{s}+\sum_{s=g_{n}(k) n+1}^{k-1} \beta_{s}^{(k)} \mathbf{g}_{s} ;$

21. Else

22. $\quad \beta_{g_{n}(k) n}^{(k)}=-\mathbf{q}_{1}^{H} \mathbf{r}_{k} / \rho_{g_{n}(k)+1} \mathbf{q}_{1}^{H} \mathbf{A g}_{g_{n}(k) n} ;$

23. $\quad$ For $s=g_{n}(k) n+1, \cdots, k-1$

24. $\quad \beta_{s}^{(k)}=-\mathbf{q}_{r_{n}(s+1)}^{H}\left(\mathbf{r}_{k}+\rho_{g_{n}(k)+1} \beta_{g_{n}(k) n}^{(k)} \mathbf{A g}_{g_{n}(k) n}+\sum_{t=g_{n}(k) n+1}^{s-1} \beta_{t}^{(k)} \mathbf{d}_{t}\right) / \mathbf{q}_{r_{n}(s+1)}^{H} \mathbf{d}_{s}$;

25. $\quad$ End

26. $\quad \mathbf{g}_{k}=\mathbf{r}_{k}+\beta_{g_{n}(k) n}^{(k)}\left(\rho_{g_{n}(k)+1} \mathbf{A}+\mathbf{I}\right) \mathbf{g}_{g_{n}(k) n}+\sum_{s=g_{n}(k) n+1}^{k-1} \beta_{s}^{(k)} \mathbf{g}_{s} ;$

27. End

28. End

We consider $\mathbf{r}_{k}$ to be the residual of the $k$ th approximate solution $\mathbf{x}_{k}$. Updating relations for $\mathbf{x}_{k}$ can be obtained from Lines 4, 5 and 9 respectively:

$$
\mathbf{x}_{k}= \begin{cases}\mathbf{x}_{k-1}-\rho_{g_{n}(k)+1} \mathbf{u}_{k}+\alpha_{k} \mathbf{g}_{k-1}, & \text { if } r_{n}(k)=1 \\ \mathbf{x}_{k-1}+\alpha_{k} \mathbf{g}_{k-1}, & \text { if } r_{n}(k)>1\end{cases}
$$

After adding (4.6) to DS\#4 and simplifying the operations appropriately, we arrive at the following $\operatorname{ML}(n)$ BiCGStab algorithm. Just like BiCGStab, the free parameter $\rho_{g_{n}}(k)+1$ on Line 5, DS\#4, is chosen to minimize the 2-norm of $\mathbf{r}_{k}$.

\section{Algorithm 4.1. $\mathrm{ML}(n)$ BiCGStab without preconditioning associated with definition (4.3)}

1. Choose an initial guess $\mathbf{x}_{0}$ and $n$ vectors $\mathbf{q}_{1}, \mathbf{q}_{2}, \cdots, \mathbf{q}_{n}$.

2. Compute $\mathbf{r}_{0}=\mathbf{b}-\mathbf{A} \mathbf{x}_{0}$ and set $\mathbf{g}_{0}=\mathbf{r}_{0}$. Compute $\mathbf{w}_{0}=\mathbf{A} \mathbf{g}_{0}, c_{0}=\mathbf{q}_{1}^{H} \mathbf{w}_{0}$.

3. For $k=1,2, \cdots$, until convergence:

4. If $r_{n}(k)=1$

5. $\quad \alpha_{k}=\mathbf{q}_{r_{n}(k)}^{H} \mathbf{r}_{k-1} / c_{k-1} ;$

6. $\quad \mathbf{u}_{k}=\mathbf{r}_{k-1}-\alpha_{k} \mathbf{w}_{k-1}$;

7. $\mathbf{x}_{k}=\mathbf{x}_{k-1}+\alpha_{k} \mathbf{g}_{k-1}$;

8. $\quad \rho_{g_{n}(k)+1}=-\left(\mathbf{A} \mathbf{u}_{k}\right)^{H} \mathbf{u}_{k} /\left\|\mathbf{A} \mathbf{u}_{k}\right\|_{2}^{2}$;

9. $\quad \mathbf{x}_{k}=\mathbf{x}_{k}-\rho_{g_{n}(k)+1} \mathbf{u}_{k}$

10. $\mathbf{r}_{k}=\rho_{g_{n}(k)+1} \mathbf{A} \mathbf{u}_{k}+\mathbf{u}_{k}$; 
11. Else

12. $\quad \widetilde{\alpha}_{k}=\mathbf{q}_{r_{n}(k)}^{H} \mathbf{u}_{k-1} / c_{k-1} ; \quad \% \widetilde{\alpha}_{k}=\alpha_{k} / \rho_{g_{n}(k-1)+1}$

13. If $r_{n}(k)<n$

14. $\quad \mathbf{u}_{k}=\mathbf{u}_{k-1}-\widetilde{\alpha}_{k} \mathbf{d}_{k-1}$;

15. End

16. $\mathbf{x}_{k}=\mathbf{x}_{k-1}+\rho_{g_{n}(k-1)+1} \widetilde{\alpha}_{k} \mathbf{g}_{k-1}$;

17. $\quad \mathbf{r}_{k}=\mathbf{r}_{k-1}-\rho_{g_{n}(k-1)+1} \widetilde{\alpha}_{k} \mathbf{w}_{k-1}$;

18. End

19. If $r_{n}(k)<n$

20. $\mathbf{z}_{d}=\mathbf{u}_{k}, \mathbf{g}_{k}=\mathbf{0}, \mathbf{z}_{w}=\mathbf{0}$;

21. For $s=k-n, \cdots, g_{n}(k) n-1$ and $g_{n}(k) \geq 1$

22. $\beta_{s}^{(k)}=-\mathbf{q}_{r_{n}(s+1)}^{H} \mathbf{z}_{d} / c_{s}$;

23. $\quad \mathbf{z}_{d}=\mathbf{z}_{d}+\beta_{s}^{(k)} \mathbf{d}_{s}$;

24. $\quad \mathbf{g}_{k}=\mathbf{g}_{k}+\beta_{s}^{(k)} \mathbf{g}_{s}$;

25. $\quad \mathbf{z}_{w}=\mathbf{z}_{w}+\beta_{s}^{(k)} \mathbf{w}_{s}$

26. End

27. $\quad \mathbf{z}_{w}=\mathbf{r}_{k}+\rho_{g_{n}(k)+1} \mathbf{z}_{w}$;

28. $\quad \tilde{\beta}_{g_{n}(k) n}^{(k)}=-\mathbf{q}_{1}^{H} \mathbf{z}_{w} / c_{g_{n}(k) n} ; \quad \% \tilde{\beta}_{g_{n}(k) n}^{(k)}=\rho_{g_{n}(k)+1} \beta_{g_{n}(k) n}^{(k)}$

29. $\quad \mathbf{z}_{w}=\mathbf{z}_{w}+\tilde{\beta}_{g_{n}(k) n}^{(k)} \mathbf{w}_{g_{n}(k) n} ;$

30. $\mathbf{g}_{k}=\mathbf{g}_{k}+\mathbf{z}_{w}+\left(\tilde{\beta}_{g_{n}(k) n}^{(k)} / \rho_{\left.g_{n}(k)+1\right)}\right) \mathbf{g}_{g_{n}(k) n} ;$

31. For $s=g_{n}(k) n+1, \cdots, k-1$

32. $\quad \beta_{s}^{(k)}=-\mathbf{q}_{r_{n}(s+1)}^{H} \mathbf{z}_{w} / c_{s}$;

33. $\quad \mathbf{g}_{k}=\mathbf{g}_{k}+\beta_{s}^{(k)} \mathbf{g}_{s}$;

34. $\quad \mathbf{z}_{w}=\mathbf{z}_{w}+\beta_{s}^{(k)} \mathbf{d}_{s}$;

35. End

36. $\mathbf{d}_{k}=\mathbf{z}_{w}-\mathbf{u}_{k}$

37. $\quad c_{k}=\mathbf{q}_{r_{n}(k+1)}^{H} \mathbf{d}_{k}$;

38. $\quad \mathbf{w}_{k}=\mathbf{A g}_{k}$;

39. Else

40. $\quad \tilde{\beta}_{g_{n}(k) n}^{(k)}=-\mathbf{q}_{1}^{H} \mathbf{r}_{k} / c_{g_{n}(k) n} ; \quad \% \tilde{\beta}_{g_{n}(k) n}^{(k)}=\rho_{g_{n}(k)+1} \beta_{g_{n}(k) n}^{(k)}$

41. $\quad \mathbf{z}_{w}=\mathbf{r}_{k}+\tilde{\beta}_{g_{n}(k) n}^{(k)} \mathbf{w}_{g_{n}(k) n} ;$

42. $\quad \mathbf{g}_{k}=\mathbf{z}_{w}+\left(\tilde{\beta}_{g_{n}(k) n}^{(k)} / \rho_{g_{n}(k)+1}\right) \mathbf{g}_{g_{n}(k) n} ;$

43. For $s=g_{n}(k) n+1, \cdots, k-1$

44. $\quad \beta_{s}^{(k)}=-\mathbf{q}_{r_{n}(s+1)}^{H} \mathbf{z}_{w} / c_{s}$;

45. $\quad \mathbf{g}_{k}=\mathbf{g}_{k}+\beta_{s}^{(k)} \mathbf{g}_{s}$;

46. $\quad \mathbf{z}_{w}=\mathbf{z}_{w}+\beta_{s}^{(k)} \mathbf{d}_{s}$;

47. End

48. $\quad \mathbf{w}_{k}=\mathbf{A} \mathbf{g}_{k}$;

49. $\quad c_{k}=\mathbf{q}_{r_{n}(k+1)}^{H} \mathbf{w}_{k}$;

50. End

51. End

Remarks: 
TABLE 4.1. Average cost per $(k$-)iteration of Algorithm 9.1 and its storage requirement.

\begin{tabular}{|c|c|c|c|}
\hline Preconditioning $\left(\mathbf{M}^{-1} \mathbf{v}\right)$ & $1+\frac{1}{n}$ & Vector addition $(\mathbf{u} \pm \mathbf{v})$ & $2-\frac{2}{n}$ \\
\hline Matvec $(\mathbf{A v})$ & $1+\frac{1}{n}$ & Saxpy $(\mathbf{u}+\alpha \mathbf{v})$ & $\max \left(2.5 n+2.5-\frac{2}{n}, 6\right)$ \\
\hline dot product $\left(\mathbf{u}^{H} \mathbf{v}\right)$ & $n+1+\frac{2}{n}$ & Storage & $\mathbf{A}+\mathbf{M}+(4 n+5) N+O(n)$ \\
\hline
\end{tabular}

(i) Algorithm 4.1 does not compute the quantities $\mathbf{u}_{k}$ and $\mathbf{d}_{k}$ when $r_{n}(k)=n$ (see Lines 13-15 and Lines 39-50).

(ii) if the $\mathbf{u}_{k}$ on Line 6 happens to be zero, then the $\rho_{g_{n}(k)+1}$ on Line 8 and therefore the $\mathbf{x}_{k}$ and $\mathbf{r}_{k}$ on Lines 9 and 10 will not be computable. In this case, however, the $\mathbf{x}_{k}$ on Line 7 will be the exact solution to system (3.1) and Algorithm 4.1] stops there.

We now compare Algorithm 4.1 with the ML( $n)$ BiCGStab algorithm in 39. First, the definitions of $\mathbf{r}_{k}, \mathbf{u}_{k}$ and $\mathbf{g}_{k}$ are the same in both algorithms, but $\mathbf{d}_{k}$ is defined differently. In [39, $\mathbf{d}_{k}=\phi_{g_{n}(k)}(\mathbf{A}) \widehat{\mathbf{g}}_{k}$. In exact arithmetic, however, both algorithms compute the same $\rho_{g_{n}(k)+1}, \mathbf{r}_{k}$ and $\mathbf{x}_{k}$. Second, the derivation of Algorithm 4.1 has been made simpler by using index functions. As a result, some redundant operations in Algorithm 2 of [39] can been seen and removed and some arithmetics are simplified. For example, the vectors $\mathbf{d}_{k}, \mathbf{u}_{k}$ are computed in every iteration in Algorithm 2 of [39]. They are now computed only when $r_{n}(k)<n$. Also, the expression of $\beta_{g_{n}(k) n}^{(k)}$ on Line 39 of Algorithm 4.1 is simpler. Some other minor changes were also made so that the algorithm becomes more efficient.

Computational cost and storage requirement of Algorithm 4.1, obtained based on its preconditioned version, Algorithm 9.1 in \$9, are summarized in Table 4.1. Since the vectors $\left\{\mathbf{q}_{1}, \ldots, \mathbf{q}_{n}\right\}$, $\left\{\mathbf{d}_{k-n}, \ldots, \mathbf{d}_{g_{n}(k) n-1}, \mathbf{d}_{g_{n}(k) n+1}, \ldots, \mathbf{d}_{k-1}\right\},\left\{\mathbf{g}_{k-n}, \ldots, \mathbf{g}_{k-1}\right\}$ and $\left\{\mathbf{w}_{k-n}, \ldots, \mathbf{w}_{g_{n}(k) n}, \mathbf{w}_{k-1}\right\}$ are required in iteration $k$, they must be stored. When $n$ is large, this storage is dominant. So, the storage requirement of the algorithm is about $4 n N$.

4.3. Properties. We summarize the properties of Algorithm 4.1 in the following proposition. Since $\mathbf{r}_{0}=\widehat{\mathbf{r}}_{0}$ by (4.4), $\nu$ (see $\oint 3.2$ ) is also the degree of the minimal polynomial of $\mathbf{r}_{0}$ with respect to $\mathbf{A}$.

Proposition 4.1. Under the assumptions of Proposition 3.1, if $\rho_{g_{n}(k)+1} \neq 0$ and $-1 / \rho_{g_{n}(k)+1} \notin$ $\sigma(\mathbf{A})$ for $1 \leq k \leq \nu-1$, where $\sigma(\mathbf{A})$ is the spectrum of $\mathbf{A}$, then Algorithm 4.1 does not break down by zero division for $k=1,2, \cdots, \nu$, and $\mathbf{x}_{\nu}$ is the exact solution of (3.1). Moreover, the computed quantities satisfy

(a) $\mathbf{x}_{k} \in \mathbf{x}_{0}+\mathcal{K}_{g_{n}(k)+k+1}\left(\mathbf{A}, \mathbf{r}_{0}\right)$ and $\mathbf{r}_{k}=\mathbf{b}-\mathbf{A} \mathbf{x}_{k} \in \mathbf{r}_{0}+\mathbf{A} \mathcal{K}_{g_{n}(k)+k+1}\left(\mathbf{A}, \mathbf{r}_{0}\right)$ for $1 \leq k \leq \nu-1$.

(b) $\mathbf{r}_{k} \neq \mathbf{0}$ for $1 \leq k \leq \nu-1$ and $\mathbf{r}_{\nu}=\mathbf{0}$.

(c) $\mathbf{r}_{k} \not \perp \mathbf{q}_{1}$ for $1 \leq k \leq \nu-1$ with $r_{n}(k)=n$.

(d) $\mathbf{u}_{k} \perp \operatorname{span}\left\{\mathbf{q}_{1}, \mathbf{q}_{2}, \cdots, \mathbf{q}_{r_{n}(k)}\right\}$ and $\mathbf{u}_{k} \not \perp \mathbf{q}_{r_{n}(k)+1}$ for $1 \leq k \leq \nu-1$ with $r_{n}(k)<n$.

(e) $\mathbf{d}_{k} \perp \operatorname{span}\left\{\mathbf{q}_{1}, \mathbf{q}_{2}, \cdots, \mathbf{q}_{r_{n}(k)}\right\}$ and $\mathbf{d}_{k} \not \perp \mathbf{q}_{r_{n}(k)+1}$ for $1 \leq k \leq \nu-1$ with $r_{n}(k)<n$.

Proof. The divisors in Algorithm 4.1 are $c_{k},\left\|\mathbf{A u}_{k}\right\|_{2}^{2}$ and $\rho_{g_{n}(k)+1}$ respectively, where the $\rho$ 's have been assumed to be nonzero. By Proposition 3.1(c), we have $\mathbf{A} \widehat{\mathbf{r}}_{k} \neq \mathbf{0}$ for $1 \leq k \leq \nu-1$. Since $-1 / \rho \notin \sigma(\mathbf{A})$ by assumption, $\phi_{g_{n}(k)}(\mathbf{A})$ is nonsingular. Hence $\mathbf{A} \mathbf{u}_{k}=\phi_{g_{n}(k)}(\mathbf{A}) \mathbf{A} \widehat{\mathbf{r}}_{k} \neq \mathbf{0}$ (see (4.3) for the first equation). Therefore, $\left\|\mathbf{A} \mathbf{u}_{k}\right\|_{2} \neq 0$ for $1 \leq k \leq \nu-1$. 
$c_{k}$ is defined respectively on Lines 37 and 49 in the algorithm. When $r_{n}(k)<n$, we have $c_{k}=$ $\mathbf{q}_{r_{n}(k+1)}^{H} \mathbf{d}_{k}$. In this case, $c_{k}=\rho_{g_{n}(k)+1} \mathbf{q}_{r_{n}(k+1)}^{H} \mathbf{A} \phi_{g_{n}(k)}(\mathbf{A}) \widehat{\mathbf{g}}_{k}=\rho_{g_{n}(k)+1} \mathbf{q}_{r_{n}(k+1)}^{H} \mathbf{A} \phi_{g_{n}(k+1)}(\mathbf{A}) \widehat{\mathbf{g}}_{k}=$ $\rho_{g_{n}(k)+1} c_{g_{n}(k+1)}^{\left(g_{n}(k+1)\right)} \mathbf{p}_{k+1}^{H} \mathbf{A} \widehat{\mathbf{g}}_{k}=\rho_{g_{n}(k)+1} c_{g_{n}(k)}^{\left(g_{n}(k)\right)} \mathbf{p}_{k+1}^{H} \mathbf{A} \widehat{\mathbf{g}}_{k}=c_{g_{n}(k)+1}^{\left(g_{n}(k)+1\right)} \mathbf{p}_{k+1}^{H} \mathbf{A} \widehat{\mathbf{g}}_{k}$ by (4.3), Proposition 2.1(d), Corollary 3.1, (4.1) and (4.2). Since the $\rho$ 's are nonzero and $\mathbf{p}_{k+1}^{H} \mathbf{A} \widehat{\mathbf{g}}_{k} \neq 0$ by Proposition $3.1(\mathrm{~g})$, we have $c_{g_{n}(k)+1}^{\left(g_{n}(k)+1\right)} \neq 0$ and hence $c_{k} \neq 0$. When $r_{n}(k)=n$, on the other hand, $c_{k}=$ $\mathbf{q}_{r_{n}(k+1)}^{H} \mathbf{w}_{k}=\mathbf{q}_{r_{n}(k+1)}^{H} \mathbf{A} \mathbf{g}_{k}$. In this case, $c_{k}=\mathbf{q}_{r_{n}(k+1)}^{H} \mathbf{A} \phi_{g_{n}(k)+1}(\mathbf{A}) \widehat{\mathbf{g}}_{k}=\mathbf{q}_{r_{n}(k+1)}^{H} \mathbf{A} \phi_{g_{n}(k+1)}(\mathbf{A}) \widehat{\mathbf{g}}_{k}$ $=c_{g_{n}(k+1)}^{\left(g_{n}(k+1)\right)} \mathbf{p}_{k+1}^{H} \mathbf{A} \widehat{\mathbf{g}}_{k}=c_{g_{n}(k)+1}^{\left(g_{n}(k)+1\right)} \mathbf{p}_{k+1}^{H} \mathbf{A} \widehat{\mathbf{g}}_{k} \neq 0$. Therefore, in either case, we always have $c_{k} \neq 0$ for $1 \leq k \leq \nu-1$. Moreover, $c_{0}=\mathbf{q}_{1}^{H} \mathbf{w}_{0}=\mathbf{q}_{1}^{H} \mathbf{A} \mathbf{g}_{0}$ according to Line 2 of the algorithm. Since $\mathbf{p}_{1}=\mathbf{q}_{1}$ by (3.2) and $\mathbf{g}_{0}=\widehat{\mathbf{g}}_{0}$ by (4.4),$c_{0} \neq 0$ by Proposition $3.1(\mathrm{~g})$.

Now that $\left\|\mathbf{A} \mathbf{u}_{k}\right\|_{2} \neq 0$ and $\rho_{g_{n}(k)+1} \neq 0$ for $1 \leq k \leq \nu-1$ and $c_{k} \neq 0$ for $0 \leq k \leq \nu-1$, Algorithm 4.1 does not break down by zero division in the first $\nu-1$ iterations. When $k=\nu$, $\mathbf{u}_{k}=\mathbf{u}_{\nu}=\phi_{g_{n}(\nu)}(\mathbf{A}) \widehat{\mathbf{r}}_{\nu}=\mathbf{0}$ and $\mathbf{r}_{k}=\mathbf{r}_{\nu}=\phi_{g_{n}(\nu)+1}(\mathbf{A}) \widehat{\mathbf{r}}_{\nu}=\mathbf{0}$ due to $\widehat{\mathbf{r}}_{\nu}=\mathbf{0}$ by Proposition 3.1. If it happens that $r_{n}(\nu)=1$, then the $\mathbf{x}_{k}\left(=\mathbf{x}_{\nu}\right)$ on Line 7 is the exact solution to system (3.1) because its residual $\mathbf{u}_{\nu}$ is zero. So, the algorithm stops there. Otherwise, the $\mathbf{x}_{k}\left(=\mathbf{x}_{\nu}\right)$ on Line 16 will be exact with residual $\mathbf{r}_{\nu}=\mathbf{0}$ and where the algorithm stops.

Part (a) follows from the definition of $\mathbf{r}_{k}$ in (4.3) and Proposition 3.1(a).

Since $\widehat{\mathbf{r}}_{k} \neq \mathbf{0}$ for $1 \leq k \leq \nu-1$ by Proposition 3.1 (b) and $\phi_{g_{n}(k)+1}(\mathbf{A})$ is nonsingular due to $-1 / \rho \notin \sigma(\mathbf{A})$, we have $\mathbf{r}_{k}=\phi_{g_{n}(k)+1}(\mathbf{A}) \widehat{\mathbf{r}}_{k} \neq \mathbf{0}$. Therefore, Part (b) holds.

For Part (c), write $k=j n+n$ with $0 \leq j$. By (4.3), (4.1) and Corollary 3.1, we have $\mathbf{q}_{1}^{H} \mathbf{r}_{k}=$ $\mathbf{q}_{1}^{H} \phi_{g_{n}(k)+1}(\mathbf{A}) \widehat{\mathbf{r}}_{k}=\mathbf{q}_{r_{n}((j+1) n+1)}^{H} \phi_{g_{n}((j+1) n+1)}(\mathbf{A}) \widehat{\mathbf{r}}_{k}=\mathbf{q}_{r_{n}(k+1)}^{H} \phi_{g_{n}(k+1)}(\mathbf{A}) \widehat{\mathbf{r}}_{k}=c_{g_{n}(k+1)}^{\left(g_{n}(k+1)\right)} \mathbf{p}_{k+1}^{H} \widehat{\mathbf{r}}_{k}=$ $c_{g_{n}(k)+1}^{\left(g_{n}(k)+1\right)} \mathbf{p}_{k+1}^{H} \widehat{\mathbf{r}}_{k}$. Now Part (c) follows from Proposition [3.1(d) and $c_{g_{n}(k)+1}^{\left(g_{n}(k)+1\right)} \neq 0$.

For the proof of Part (d), we first note that Algorithm 4.1 does not compute $\mathbf{u}_{k}$ when $r_{n}(k)=n$ (see Lines 13 - 15). Write $k=j n+i$ as in (2.1) and let $1 \leq t \leq i<n$. Then $r_{n}(k)=i, g_{n}(k)=j=$ $g_{n}(j n+t)$ and $r_{n}(j n+t)=t$. Now, by (4.3) and Corollary 3.1, we have $\mathbf{q}_{t}^{H} \mathbf{u}_{k}=\mathbf{q}_{t}^{H} \phi_{g_{n}(k)}(\mathbf{A}) \widehat{\mathbf{r}}_{k}=$ $\mathbf{q}_{r_{n}(j n+t)}^{H} \phi_{g_{n}(j n+t)}(\mathbf{A}) \widehat{\mathbf{r}}_{k}=c_{g_{n}(j n+t)}^{\left(g_{n}(j n+t)\right)} \mathbf{p}_{j n+t}^{H} \widehat{\mathbf{r}}_{k}$. Since $\mathbf{p}_{j n+t}^{H} \widehat{\mathbf{r}}_{k}=0$ by Proposition $3.1(\mathrm{~d}), \mathbf{q}_{t}^{H} \mathbf{u}_{k}=0$ for $1 \leq t \leq i$. Similarly, $\mathbf{q}_{i+1}^{H} \mathbf{u}_{k}=c_{g_{n}(k)}^{\left(g_{n}(k)\right)} \mathbf{p}_{j n+i+1}^{H} \widehat{\mathbf{r}}_{k}=c_{g_{n}(k)}^{\left(g_{n}(k)\right)} \mathbf{p}_{k+1}^{H} \widehat{\mathbf{r}}_{k}$ (the validity of the first equation requires $i<n)$. Because of Proposition $\underline{3.1}(\mathrm{~d})$ and $c_{g_{n}(k)}^{\left(g_{n}(k)\right)} \neq 0, \mathbf{q}_{i+1}^{H} \mathbf{u}_{k} \neq 0$.

Similar to the quantity $\mathbf{u}_{k}$, Algorithm 4.1 does not compute $\mathbf{d}_{k}$ when $r_{n}(k)=n$ (see Lines 40 - 49). By (4.3), $\mathbf{d}_{k}=\rho_{g_{n}(k)+1} \mathbf{A} \phi_{g_{n}(k)}(\mathbf{A}) \widehat{\mathbf{g}}_{k}$ and the proof of Part (e) is parallel to that of Part (d).

The conditions of $\rho_{g_{n}(k)+1} \neq 0$ and $-1 / \rho_{g_{n}(k)+1} \notin \sigma(\mathbf{A})$ can be easily made satisfied. For example, one can add some small random noise (e.g., $N(0, \delta)$ with $\delta \ll 1)$ to $\rho_{g_{n}(k)+1}$ after it is computed.

Corollary 4.1. Consider the case where $n=1$, (3.1) is a real system and $\mathbf{q}_{1} \in \mathcal{R}^{N}$ is a random vector with iid elements from $N(0,1)$. If some small random number is added to $\rho_{g_{n}(k)+1}$ after it is computed so that $\rho_{g_{n}(k)+1} \neq 0$ and $-1 / \rho_{g_{n}(k)+1} \notin \sigma(\mathbf{A})$, then Algorithm 4.1] will work almost surely without breakdown by zero division to find a solution of (3.1) from the affine space $\mathbf{x}_{0}+$ $\operatorname{span}\left\{\mathbf{A}^{t} \mathbf{r}_{0} \mid t \in \mathcal{N}_{0}\right\}$ provided that $\mathbf{x}_{0} \in \mathcal{R}^{N}$ is chosen such that the affine space contains a solution to (3.1).

Proposition 4.1 indicates that exact solution can only be found at iteration $k=\nu$. It is possible, however, that $\left\|\mathbf{r}_{k}\right\|_{2}$ can become very small for some $k<\nu$. In practice, we terminate the algorithm when $\left\|\mathbf{r}_{k}\right\|_{2}$ falls within a given tolerance. 
As in the case of BiCGStab, ML $(n)$ BiCGStab can encounter a breakdown in its implementation. $\mathrm{ML}(n) \mathrm{BiCGStab}$, besides the two types of breakdown of $\mathrm{ML}(n) \mathrm{BiCG}$, has one more type of breakdown caused by $\rho_{g_{n}(k)+1}$. In more detail, the divisors in Algorithm 4.1 are $c_{k},\left\|\mathbf{A} \mathbf{u}_{k}\right\|_{2}^{2}$ and $\rho_{g_{n}(k)+1}$. If $\left\|\mathbf{A} \mathbf{u}_{k}\right\|_{2}=0$, then $\rho_{g_{n}(k)+1}=\infty$ and a breakdown due to the overflow of $\rho_{g_{n}(k)+1}$ occurs. Under the assumptions of Proposition 4.1, on the other hand, it can be shown (see the proof of the proposition) that $c_{k}=c_{g_{n}(k)+1}^{\left(g_{n}(k)+1\right)} \mathbf{p}_{k+1}^{H} \mathbf{A} \widehat{\mathbf{g}}_{k}$, where $c_{g_{n}(k)+1}^{\left(g_{n}(k)+1\right)}$ is the leading coefficient of $\phi_{g_{n}(k)+1}(\lambda)$ (see (4.1)). So, $c_{k}$ is a quantity that relates to $\rho_{g_{n}(k)+1}$ and the $\operatorname{ML}(n) \operatorname{BiCG}$ divisor $\mathbf{p}_{k+1}^{H} \mathbf{A} \widehat{\mathbf{g}}_{k}$. Thus, either $\rho_{g_{n}(k)+1}=0$ or $\mathbf{p}_{k+1}^{H} \mathbf{A} \widehat{\mathbf{g}}_{k}=0$ can cause $c_{k}=0$.

\section{A Second $\operatorname{ML}(n)$ BiCGStab Algorithm}

If we write $k=j n+i$ as in (2.1), the $\mathbf{r}_{k}$ defined by (4.4) then becomes

$$
\mathbf{r}_{j n+i}=\phi_{j+1}(\mathbf{A}) \widehat{\mathbf{r}}_{j n+i}
$$

where $i=1,2, \cdots, n$ and $j=0,1,2, \cdots$.

Starting with $k=1$, let us call every $n$ consecutive $k$-iterations a "cycle", namely, iterations $k=1,2, \cdots, n$ form the first cycle, iterations $k=n+1, n+2, \cdots, n+n$ the second cycle and so on. Then (5.1) increases the degree of the polynomial $\phi$ by 1 at the beginning of every cycle. For example, consider $n=3$. Then (5.1) implies that

$$
\begin{array}{lll}
\mathbf{r}_{1}=\phi_{1}(\mathbf{A}) \widehat{\mathbf{r}}_{1}, & \mathbf{r}_{4}=\phi_{2}(\mathbf{A}) \widehat{\mathbf{r}}_{4}, & \mathbf{r}_{7}=\phi_{3}(\mathbf{A}) \widehat{\mathbf{r}}_{7} \\
\mathbf{r}_{2}=\phi_{1}(\mathbf{A}) \widehat{\mathbf{r}}_{2}, & \mathbf{r}_{5}=\phi_{2}(\mathbf{A}) \widehat{\mathbf{r}}_{5}, & \mathbf{r}_{8}=\phi_{3}(\mathbf{A}) \widehat{\mathbf{r}}_{8}, \\
\mathbf{r}_{3}=\phi_{1}(\mathbf{A}) \widehat{\mathbf{r}}_{3}, & \mathbf{r}_{6}=\phi_{2}(\mathbf{A}) \widehat{\mathbf{r}}_{6}, & \mathbf{r}_{9}=\phi_{3}(\mathbf{A}) \widehat{\mathbf{r}}_{9} .
\end{array}
$$

Iteration $k=4$ is the first iteration of the second cycle and the degree of $\phi$ is increased from 1 to 2 there.

One can define $\mathbf{r}_{k}$ by increasing the degree of $\phi$ by one anywhere within a cycle. Correspondingly, (we believe) the definition will lead to a different algorithm of $\mathrm{ML}(n) \operatorname{BiCGStab.~As~an~}$ illustration, let us increase the degree of $\phi$ at the end of every cycle and derive the algorithm associated with it.

5.1. Notation and Definitions. Let $\phi_{k}(\lambda)$ be defined as in (1.1). For $k \in \mathcal{N}$, define

$$
\begin{array}{ll}
\mathbf{r}_{k}=\phi_{g_{n}(k+1)}(\mathbf{A}) \widehat{\mathbf{r}}_{k}, & \mathbf{g}_{k}=\phi_{g_{n}(k+1)}(\mathbf{A}) \widehat{\mathbf{g}}_{k}, \\
\mathbf{u}_{k}=\phi_{g_{n}(k)}(\mathbf{A}) \widehat{\mathbf{r}}_{k}, & \mathbf{w}_{k}=\mathbf{A g}_{k} .
\end{array}
$$

and set

$$
\mathbf{r}_{0}=\widehat{\mathbf{r}}_{0} \quad \text { and } \quad \mathbf{g}_{0}=\widehat{\mathbf{g}}_{0} .
$$

The vector $\mathbf{r}_{k}$ is considered to be the residual of the approximate solution $\mathbf{x}_{k}$ computed. We remark that $\mathbf{r}_{k}=\mathbf{u}_{k}$ when $r_{n}(k)<n$ since $g_{n}(k+1)=g_{n}(k)$ in this case.

Definition (5.2) increases the degree of $\phi$ at the end of a cycle. To see this, let $n=3$. Then (5.2) yields

$$
\begin{array}{lll}
\mathbf{r}_{1}=\phi_{0}(\mathbf{A}) \widehat{\mathbf{r}}_{1}, & \mathbf{r}_{4}=\phi_{1}(\mathbf{A}) \widehat{\mathbf{r}}_{4}, & \mathbf{r}_{7}=\phi_{2}(\mathbf{A}) \widehat{\mathbf{r}}_{7} \\
\mathbf{r}_{2}=\phi_{0}(\mathbf{A}) \widehat{\mathbf{r}}_{2}, & \mathbf{r}_{5}=\phi_{1}(\mathbf{A}) \widehat{\mathbf{r}}_{5}, & \mathbf{r}_{8}=\phi_{2}(\mathbf{A}) \widehat{\mathbf{r}}_{8}, \\
\mathbf{r}_{3}=\phi_{1}(\mathbf{A}) \widehat{\mathbf{r}}_{3}, & \mathbf{r}_{6}=\phi_{2}(\mathbf{A}) \widehat{\mathbf{r}}_{6}, & \mathbf{r}_{9}=\phi_{3}(\mathbf{A}) \widehat{\mathbf{r}}_{9} .
\end{array}
$$

5.2. Algorithm Derivation. To derive the algorithm associated with (5.2), we first transform Algorithm 3.1 (forgetting Lines 1, 2, 5 and 11) into the following version which is computationally equivalent to Algorithm 3.1, but is more convenient for us to apply Proposition 2.1.

Derivation Stage \#5. 
1. For $k=1,2, \cdots$, until convergence:

2. $\alpha_{k}=\mathbf{p}_{k}^{H} \widehat{\mathbf{r}}_{k-1} / \mathbf{p}_{k}^{H} \mathbf{A} \widehat{\mathbf{g}}_{k-1} ;$

3. If $r_{n}(k)<n$

4. $\quad \widehat{\mathbf{r}}_{k}=\widehat{\mathbf{r}}_{k-1}-\alpha_{k} \mathbf{A} \widehat{\mathbf{g}}_{k-1}$;

5. $\quad$ For $s=\max (k-n, 0), \cdots, g_{n}(k) n-1$

6. $\quad \beta_{s}^{(k)}=-\mathbf{p}_{s+1}^{H} \mathbf{A}\left(\widehat{\mathbf{r}}_{k}+\sum_{t=\max (k-n, 0)}^{s-1} \beta_{t}^{(k)} \widehat{\mathbf{g}}_{t}\right) / \mathbf{p}_{s+1}^{H} \mathbf{A} \widehat{\mathbf{g}}_{s} ;$

7. $\quad$ End

8. $\quad$ For $s=g_{n}(k) n, \cdots, k-1$

9. $\quad \beta_{s}^{(k)}=-\mathbf{p}_{s+1}^{H} \mathbf{A}\left(\widehat{\mathbf{r}}_{k}+\sum_{t=\max (k-n, 0)}^{g_{n}(k) n-1} \beta_{t}^{(k)} \widehat{\mathbf{g}}_{t}+\sum_{t=g_{n}(k) n}^{s-1} \beta_{t}^{(k)} \widehat{\mathbf{g}}_{t}\right) / \mathbf{p}_{s+1}^{H} \mathbf{A} \widehat{\mathbf{g}}_{s} ;$

10. $\quad$ End

11. $\widehat{\mathbf{g}}_{k}=\widehat{\mathbf{r}}_{k}+\sum_{s=\max (k-n, 0)}^{g_{n}(k) n-1} \beta_{s}^{(k)} \widehat{\mathbf{g}}_{s}+\sum_{s=g_{n}(k) n}^{k-1} \beta_{s}^{(k)} \widehat{\mathbf{g}}_{s} ;$

12. Else

13. $\quad \widehat{\mathbf{r}}_{k}=\widehat{\mathbf{r}}_{k-1}-\alpha_{k} \mathbf{A} \widehat{\mathbf{g}}_{k-1} ;$

14. $\quad$ For $s=g_{n}(k) n, \cdots, k-1$

15. $\beta_{s}^{(k)}=-\mathbf{p}_{s+1}^{H} \mathbf{A}\left(\widehat{\mathbf{r}}_{k}+\sum_{t=g_{n}(k) n}^{s-1} \beta_{t}^{(k)} \widehat{\mathbf{g}}_{t}\right) / \mathbf{p}_{s+1}^{H} \mathbf{A} \widehat{\mathbf{g}}_{s} ;$

16. $\quad$ End

17. $\widehat{\mathbf{g}}_{k}=\widehat{\mathbf{r}}_{k}+\sum_{s=g_{n}(k) n}^{k-1} \beta_{s}^{(k)} \widehat{\mathbf{g}}_{s} ;$

18. End

19. End

Then we transform DS\#5 as follows by Corollary 3.1 .

\section{Derivation Stage \#6.}

1. For $k=1,2, \cdots$, until convergence:

2. $\quad \alpha_{k}=\mathbf{q}_{r_{n}(k)}^{H} \phi_{g_{n}(k)}(\mathbf{A}) \widehat{\mathbf{r}}_{k-1} / \mathbf{q}_{r_{n}(k)}^{H} \mathbf{A} \phi_{g_{n}(k)}(\mathbf{A}) \widehat{\mathbf{g}}_{k-1} ;$

3. If $r_{n}(k)<n$

4. $\quad \phi_{g_{n}(k)}(\mathbf{A}) \widehat{\mathbf{r}}_{k}=\phi_{g_{n}(k)}(\mathbf{A}) \widehat{\mathbf{r}}_{k-1}-\alpha_{k} \mathbf{A} \phi_{g_{n}(k)}(\mathbf{A}) \widehat{\mathbf{g}}_{k-1}$

5. $\quad$ For $s=\max (k-n, 0), \cdots, g_{n}(k) n-1$

6. $\quad \beta_{s}^{(k)}=-\mathbf{q}_{r_{n}(s+1)}^{H}\left(\phi_{g_{n}(s+1)+1}(\mathbf{A}) \widehat{\mathbf{r}}_{k}\right.$

$\left.+\rho_{g_{n}(s+1)+1} \sum_{t=\max (k-n, 0)}^{s-1} \beta_{t}^{(k)} \mathbf{A} \phi_{g_{n}(s+1)}(\mathbf{A}) \widehat{\mathbf{g}}_{t}\right) / \rho_{g_{n}(s+1)+1} \mathbf{q}_{r_{n}(s+1)}^{H} \mathbf{A} \phi_{g_{n}(s+1)}(\mathbf{A}) \widehat{\mathbf{g}}_{s} ;$

7. $\quad$ End

8. $\quad$ For $s=g_{n}(k) n, \cdots, k-1$

9. $\quad \beta_{s}^{(k)}=-\mathbf{q}_{r_{n}(s+1)}^{H} \mathbf{A}\left(\phi_{g_{n}(s+1)}(\mathbf{A}) \widehat{\mathbf{r}}_{k}+\sum_{t=\max (k-n, 0)}^{g_{n}(k) n-1} \beta_{t}^{(k)} \phi_{g_{n}(s+1)}(\mathbf{A}) \widehat{\mathbf{g}}_{t}\right.$ $\left.+\sum_{t=g_{n}(k) n}^{s-1} \beta_{t}^{(k)} \phi_{g_{n}(s+1)}(\mathbf{A}) \widehat{\mathbf{g}}_{t}\right) / \mathbf{q}_{r_{n}(s+1)}^{H} \mathbf{A} \phi_{g_{n}(s+1)}(\mathbf{A}) \widehat{\mathbf{g}}_{s}$;

10. $\quad$ End

11. $\quad \phi_{g_{n}(k+1)}(\mathbf{A}) \widehat{\mathbf{g}}_{k}=\phi_{g_{n}(k+1)}(\mathbf{A}) \widehat{\mathbf{r}}_{k}+\left(\rho_{g_{n}(k+1)} \mathbf{A}+\mathbf{I}\right) \sum_{s=\max (k-n, 0)}^{g_{n}(k) n-1} \beta_{s}^{(k)} \phi_{g_{n}(k+1)-1}(\mathbf{A}) \widehat{\mathbf{g}}_{s}+$ $\sum_{s=g_{n}(k) n}^{k-1} \beta_{s}^{(k)} \phi_{g_{n}(k+1)}(\mathbf{A}) \widehat{\mathbf{g}}_{s}$;

12. Else

13. $\quad \phi_{g_{n}(k)}(\mathbf{A}) \widehat{\mathbf{r}}_{k}=\phi_{g_{n}(k)}(\mathbf{A}) \widehat{\mathbf{r}}_{k-1}-\alpha_{k} \mathbf{A} \phi_{g_{n}(k)}(\mathbf{A}) \widehat{\mathbf{g}}_{k-1} ;$

14. $\quad \phi_{g_{n}(k+1)}(\mathbf{A}) \widehat{\mathbf{r}}_{k}=\left(\rho_{g_{n}(k+1)} \mathbf{A}+\mathbf{I}\right) \phi_{g_{n}(k+1)-1}(\mathbf{A}) \widehat{\mathbf{r}}_{k}$;

15. $\quad$ For $s=g_{n}(k) n, \cdots, k-1$

16. $\quad \beta_{s}^{(k)}=-\mathbf{q}_{r_{n}(s+1)}^{H}\left(\phi_{g_{n}(s+1)+1}(\mathbf{A}) \widehat{\mathbf{r}}_{k}+\right.$ 
17.

$$
\left.\rho_{g_{n}(s+1)+1} \sum_{t=g_{n}(k) n}^{s-1} \beta_{t}^{(k)} \mathbf{A} \phi_{g_{n}(s+1)}(\mathbf{A}) \widehat{\mathbf{g}}_{t}\right) / \rho_{g_{n}(s+1)+1} \mathbf{q}_{r_{n}(s+1)}^{H} \mathbf{A} \phi_{g_{n}(s+1)}(\mathbf{A}) \widehat{\mathbf{g}}_{s} ;
$$

18. $\quad \phi_{g_{n}(k+1)}(\mathbf{A}) \widehat{\mathbf{g}}_{k}=\phi_{g_{n}(k+1)}(\mathbf{A}) \widehat{\mathbf{r}}_{k}+\left(\rho_{g_{n}(k+1)} \mathbf{A}+\mathbf{I}\right) \sum_{s=g_{n}(k) n}^{k-1} \beta_{s}^{(k)} \phi_{g_{n}(k+1)-1}(\mathbf{A}) \widehat{\mathbf{g}}_{s} ;$

19. End

20. End

Lines 4, 11, 13 and 18, DS\#6, were obtained from Lines 4, 11, 13 and 17, DS\#5, by multiplying them with $\phi_{g_{n}(k)}(\mathbf{A})$ and $\phi_{g_{n}(k+1)}(\mathbf{A})$ respectively. Line 14, DS\#6, is a direct result of the definition (1.1) of $\phi$.

Now we use Proposition 2.1 to write DS\#6 as

\section{Derivation Stage \#7.}

1. For $k=1,2, \cdots$, until convergence:

2. $\quad \alpha_{k}=\mathbf{q}_{r_{n}(k)}^{H} \phi_{g_{n}(k)}(\mathbf{A}) \widehat{\mathbf{r}}_{k-1} / \mathbf{q}_{r_{n}(k)}^{H} \mathbf{A} \phi_{g_{n}(k)}(\mathbf{A}) \widehat{\mathbf{g}}_{k-1} ;$

3. If $r_{n}(k)<n$

4. $\quad \phi_{g_{n}(k+1)}(\mathbf{A}) \widehat{\mathbf{r}}_{k}=\phi_{g_{n}(k)}(\mathbf{A}) \widehat{\mathbf{r}}_{k-1}-\alpha_{k} \mathbf{A} \phi_{g_{n}(k)}(\mathbf{A}) \widehat{\mathbf{g}}_{k-1}$;

5. $\quad$ For $s=\max (k-n, 0), \cdots, g_{n}(k) n-1$

6. $\quad \beta_{s}^{(k)}=-\mathbf{q}_{r_{n}(s+1)}^{H}\left(\phi_{g_{n}(k+1)}(\mathbf{A}) \widehat{\mathbf{r}}_{k}\right.$

$\left.+\rho_{g_{n}(k+1)} \sum_{t=\max (k-n, 0)}^{s-1} \beta_{t}^{(k)} \mathbf{A} \phi_{g_{n}(t+1)}(\mathbf{A}) \widehat{\mathbf{g}}_{t}\right) / \rho_{g_{n}(k+1)} \mathbf{q}_{r_{n}(s+1)}^{H} \mathbf{A} \phi_{g_{n}(s+1)}(\mathbf{A}) \widehat{\mathbf{g}}_{s} ;$

7. $\quad$ End

8. $\quad$ For $s=g_{n}(k) n, \cdots, k-1$

9

$$
\begin{aligned}
\beta_{s}^{(k)}= & -\mathbf{q}_{r_{n}(s+1)}^{H} \mathbf{A}\left(\phi_{g_{n}(k+1)}(\mathbf{A}) \widehat{\mathbf{r}}_{k}+\sum_{t=\max (k-n, 0)}^{g_{n}(k) n-1} \beta_{t}^{(k)} \phi_{g_{n}(t+1)+1}(\mathbf{A}) \widehat{\mathbf{g}}_{t}\right. \\
& \left.+\sum_{t=g_{n}(k) n}^{s-1} \beta_{t}^{(k)} \phi_{g_{n}(t+1)}(\mathbf{A}) \widehat{\mathbf{g}}_{t}\right) / \mathbf{q}_{r_{n}(s+1)}^{H} \mathbf{A} \phi_{g_{n}(s+1)}(\mathbf{A}) \widehat{\mathbf{g}}_{s} ;
\end{aligned}
$$

10. $\quad$ End

11. $\quad \phi_{g_{n}(k+1)}(\mathbf{A}) \widehat{\mathbf{g}}_{k}=\phi_{g_{n}(k+1)}(\mathbf{A}) \widehat{\mathbf{r}}_{k}+\left(\rho_{g_{n}(k+1)} \mathbf{A}+\mathbf{I}\right) \sum_{s=\max (k-n, 0)}^{g_{n}(k) n-1} \beta_{s}^{(k)} \phi_{g_{n}(s+1)}(\mathbf{A}) \widehat{\mathbf{g}}_{s}+$ $\sum_{s=g_{n}(k) n}^{k-1} \beta_{s}^{(k)} \phi_{g_{n}(s+1)}(\mathbf{A}) \widehat{\mathbf{g}}_{s}$;

12. Else

13. $\quad \phi_{g_{n}(k)}(\mathbf{A}) \widehat{\mathbf{r}}_{k}=\phi_{g_{n}(k)}(\mathbf{A}) \widehat{\mathbf{r}}_{k-1}-\alpha_{k} \mathbf{A} \phi_{g_{n}(k)}(\mathbf{A}) \widehat{\mathbf{g}}_{k-1} ;$

14. $\quad \phi_{g_{n}(k+1)}(\mathbf{A}) \widehat{\mathbf{r}}_{k}=\left(\rho_{g_{n}(k+1)} \mathbf{A}+\mathbf{I}\right) \phi_{g_{n}(k)}(\mathbf{A}) \widehat{\mathbf{r}}_{k}$;

15. $\quad$ For $s=g_{n}(k) n, \cdots, k-1$

16. $\quad \beta_{s}^{(k)}=-\mathbf{q}_{r_{n}(s+1)}^{H}\left(\phi_{g_{n}(k+1)}(\mathbf{A}) \widehat{\mathbf{r}}_{k}+\right.$

17. $\quad$ End

$$
\left.\rho_{g_{n}(k+1)} \sum_{t=g_{n}(k) n}^{s-1} \beta_{t}^{(k)} \mathbf{A} \phi_{g_{n}(t+1)}(\mathbf{A}) \widehat{\mathbf{g}}_{t}\right) / \rho_{g_{n}(k+1)} \mathbf{q}_{r_{n}(s+1)}^{H} \mathbf{A} \phi_{g_{n}(s+1)}(\mathbf{A}) \widehat{\mathbf{g}}_{s} ;
$$

18. $\quad \phi_{g_{n}(k+1)}(\mathbf{A}) \widehat{\mathbf{g}}_{k}=\phi_{g_{n}(k+1)}(\mathbf{A}) \widehat{\mathbf{r}}_{k}+\left(\rho_{g_{n}(k+1)} \mathbf{A}+\mathbf{I}\right) \sum_{s=g_{n}(k) n}^{k-1} \beta_{s}^{(k)} \phi_{g_{n}(s+1)}(\mathbf{A}) \widehat{\mathbf{g}}_{s} ;$

19. End

20. End

We remark that the term $\phi_{g_{n}(t+1)+1}(\mathbf{A}) \widehat{\mathbf{g}}_{t}$ in the first sum on Line 9 can be further written as

$$
\begin{aligned}
\phi_{g_{n}(t+1)+1}(\mathbf{A}) \widehat{\mathbf{g}}_{t} & =\left(\rho_{g_{n}(t+1)+1} \mathbf{A}+\mathbf{I}\right) \phi_{g_{n}(t+1)}(\mathbf{A}) \widehat{\mathbf{g}}_{t} \\
& =\left(\rho_{g_{n}(k+1)} \mathbf{A}+\mathbf{I}\right) \phi_{g_{n}(t+1)}(\mathbf{A}) \widehat{\mathbf{g}}_{t} .
\end{aligned}
$$

Substituting (5.4) and (5.2) into DS\#7 then yields a set of updating relations of the vectors defined by (5.2). 


\section{Derivation Stage \#8.}

1. For $k=1,2, \cdots$, until convergence:

2. $\quad \alpha_{k}=\mathbf{q}_{r_{n}(k)}^{H} \mathbf{r}_{k-1} / \mathbf{q}_{r_{n}(k)}^{H} \mathbf{w}_{k-1}$

3. If $r_{n}(k)<n$

4. $\quad \mathbf{r}_{k}=\mathbf{r}_{k-1}-\alpha_{k} \mathbf{w}_{k-1}$

5. $\quad$ For $s=\max (k-n, 0), \cdots, g_{n}(k) n-1$

6. $\quad \beta_{s}^{(k)}=-\mathbf{q}_{r_{n}(s+1)}^{H}\left(\mathbf{r}_{k}+\rho_{g_{n}(k+1)} \sum_{t=\max (k-n, 0)}^{s-1} \beta_{t}^{(k)} \mathbf{w}_{t}\right) / \rho_{g_{n}(k+1)} \mathbf{q}_{r_{n}(s+1)}^{H} \mathbf{w}_{s} ;$

7. $\quad$ End

8. $\quad$ For $s=g_{n}(k) n, \cdots, k-1$

9. $\quad \beta_{s}^{(k)}=-\mathbf{q}_{r_{n}(s+1)}^{H}\left(\mathbf{A r}_{k}+\sum_{t=\max (k-n, 0)}^{g_{n}(k) n-1} \beta_{t}^{(k)}\left(\rho_{g_{n}(k+1)} \mathbf{A}+\mathbf{I}\right) \mathbf{w}_{t}\right.$ $\left.+\sum_{t=g_{n}(k) n}^{s-1} \beta_{t}^{(k)} \mathbf{w}_{t}\right) / \mathbf{q}_{r_{n}(s+1)}^{H} \mathbf{w}_{s}$;

10. $\quad$ End

11. $\quad \mathbf{g}_{k}=\mathbf{r}_{k}+\rho_{g_{n}(k+1)} \sum_{s=\max (k-n, 0)}^{g_{n}(k) n-1} \beta_{s}^{(k)} \mathbf{w}_{s}+\sum_{s=\max (k-n, 0)}^{g_{n}(k) n-1} \beta_{s}^{(k)} \mathbf{g}_{s}+\sum_{s=g_{n}(k) n}^{k-1} \beta_{s}^{(k)} \mathbf{g}_{s} ;$

12. Else

13. $\mathbf{u}_{k}=\mathbf{r}_{k-1}-\alpha_{k} \mathbf{w}_{k-1}$;

14. $\quad \mathbf{r}_{k}=\left(\rho_{g_{n}(k+1)} \mathbf{A}+\mathbf{I}\right) \mathbf{u}_{k}$

15. For $s=g_{n}(k) n, \cdots, k-1$

16. $\quad \beta_{s}^{(k)}=-\mathbf{q}_{r_{n}(s+1)}^{H}\left(\mathbf{r}_{k}+\rho_{g_{n}(k+1)} \sum_{t=g_{n}(k) n}^{s-1} \beta_{t}^{(k)} \mathbf{w}_{t}\right) / \rho_{g_{n}(k+1)} \mathbf{q}_{r_{n}(s+1)}^{H} \mathbf{w}_{s} ;$

17. $\quad$ End

18. $\quad \mathbf{g}_{k}=\mathbf{r}_{k}+\rho_{g_{n}(k+1)} \sum_{s=g_{n}(k) n}^{k-1} \beta_{s}^{(k)} \mathbf{w}_{s}+\sum_{s=g_{n}(k) n}^{k-1} \beta_{s}^{(k)} \mathbf{g}_{s} ;$

19. End

20. End

DS\#8 does not contain any update about $\mathbf{w}_{k}$. For the updates, we multiply the equations on Lines 11 and 18 by $\mathbf{A}$ to get

$$
\begin{aligned}
\mathbf{w}_{k}= & \mathbf{A}\left(\mathbf{r}_{k}+\rho_{g_{n}(k+1)} \sum_{s=\max (k-n, 0)}^{g_{n}(k) n-1} \beta_{s}^{(k)} \mathbf{w}_{s}\right)+\sum_{s=\max (k-n, 0)}^{g_{n}(k) n-1} \beta_{s}^{(k)} \mathbf{w}_{s} \\
& +\sum_{s=g_{n}(k) n}^{k-1} \beta_{s}^{(k)} \mathbf{w}_{s}
\end{aligned}
$$

if $r_{n}(k)<n$, and

$$
\mathbf{w}_{k}=\mathbf{A}\left(\mathbf{r}_{k}+\rho_{g_{n}(k+1)} \sum_{s=g_{n}(k) n}^{k-1} \beta_{s}^{(k)} \mathbf{w}_{s}\right)+\sum_{s=g_{n}(k) n}^{k-1} \beta_{s}^{(k)} \mathbf{w}_{s}
$$

if $r_{n}(k)=n$.

Again, we consider $\mathbf{r}_{k}$ to be a residual. To be consistent with Lines 4, 13 and 14, we update the solution vector $\mathbf{x}_{k}$ as

$$
\mathbf{x}_{k}= \begin{cases}\mathbf{x}_{k-1}+\alpha_{k} \mathbf{g}_{k-1}, & \text { if } r_{n}(k)<n \\ -\rho_{g_{n}(k+1)} \mathbf{u}_{k}+\mathbf{x}_{k-1}+\alpha_{k} \mathbf{g}_{k-1}, & \text { if } r_{n}(k)=n .\end{cases}
$$

Now adding (5.5), (5.6) and (5.7) to DS\#8 and simplifying the operations appropriately, we then arrive at the following algorithm. The free parameter $\rho_{g_{n}}(k+1)$ is chosen to minimize the 2-norm of $\mathbf{r}_{k}$.

\section{Algorithm 5.1. ML $(n)$ BiCGStab without preconditioning associated with definition (5.2)}


1. Choose an initial guess $\mathbf{x}_{0}$ and $n$ vectors $\mathbf{q}_{1}, \mathbf{q}_{2}, \cdots, \mathbf{q}_{n}$.

2. Compute $\mathbf{r}_{0}=\mathbf{b}-\mathbf{A} \mathbf{x}_{0}$ and $\mathbf{g}_{0}=\mathbf{r}_{0}, \mathbf{w}_{0}=\mathbf{A g}_{0}, c_{0}=\mathbf{q}_{1}^{H} \mathbf{w}_{0}$.

3. For $k=1,2, \cdots$, until convergence:

4. $\quad \alpha_{k}=\mathbf{q}_{r_{n}(k)}^{H} \mathbf{r}_{k-1} / c_{k-1}$;

5. If $r_{n}(k)<n$

6. $\quad \mathbf{x}_{k}=\mathbf{x}_{k-1}+\alpha_{k} \mathbf{g}_{k-1}$;

7. $\quad \mathbf{r}_{k}=\mathbf{r}_{k-1}-\alpha_{k} \mathbf{w}_{k-1}$;

8. $\quad \mathbf{z}_{w}=\mathbf{r}_{k}, \mathbf{g}_{k}=\mathbf{0}$;

9. $\quad$ For $s=\max (k-n, 0), \cdots, g_{n}(k) n-1$

10. $\tilde{\beta}_{s}^{(k)}=-\mathbf{q}_{r_{n}(s+1)}^{H} \mathbf{z}_{w} / c_{s} ; \quad \% \tilde{\beta}_{s}^{(k)}=\beta_{s}^{(k)} \rho_{g_{n}(k+1)}$

11. $\quad \mathbf{z}_{w}=\mathbf{z}_{w}+\tilde{\beta}_{s}^{(k)} \mathbf{w}_{s}$;

12. $\mathbf{g}_{k}=\mathbf{g}_{k}+\tilde{\beta}_{s}^{(k)} \mathbf{g}_{s}$;

13. End

14. $\quad \mathbf{g}_{k}=\mathbf{z}_{w}+\frac{1}{\rho_{g_{n}(k+1)}} \mathbf{g}_{k}$;

15. $\mathbf{w}_{k}=\mathbf{A g}_{k}$;

16. For $s=g_{n}(k) n, \cdots, k-1$

17. $\quad \beta_{s}^{(k)}=-\mathbf{q}_{r_{n}(s+1)}^{H} \mathbf{w}_{k} / c_{s}$;

18. $\quad \mathbf{w}_{k}=\mathbf{w}_{k}+\beta_{s}^{(k)} \mathbf{w}_{s}$;

19. $\quad \mathbf{g}_{k}=\mathbf{g}_{k}+\beta_{s}^{(k)} \mathbf{g}_{s}$;

20. End

21. Else

22. $\mathbf{x}_{k}=\mathbf{x}_{k-1}+\alpha_{k} \mathbf{g}_{k-1}$;

23. $\mathbf{u}_{k}=\mathbf{r}_{k-1}-\alpha_{k} \mathbf{w}_{k-1}$;

24. $\quad \rho_{g_{n}(k+1)}=-\left(\mathbf{A} \mathbf{u}_{k}\right)^{H} \mathbf{u}_{k} /\left\|\mathbf{A} \mathbf{u}_{k}\right\|_{2}^{2}$;

25. $\mathbf{x}_{k}=\mathbf{x}_{k}-\rho_{g_{n}(k+1)} \mathbf{u}_{k}$;

26. $\quad \mathbf{r}_{k}=\rho_{g_{n}(k+1)} \mathbf{A} \mathbf{u}_{k}+\mathbf{u}_{k}$;

27. $\quad \mathbf{z}_{w}=\mathbf{r}_{k}, \mathbf{g}_{k}=\mathbf{0}$;

28. For $s=g_{n}(k) n, \cdots, k-1$

29. $\quad \tilde{\beta}_{s}^{(k)}=-\mathbf{q}_{r_{n}(s+1)}^{H} \mathbf{z}_{w} / c_{s} ; \quad \% \tilde{\beta}_{s}^{(k)}=\beta_{s}^{(k)} \rho_{g_{n}(k+1)}$

30. $\quad \mathbf{z}_{w}=\mathbf{z}_{w}+\tilde{\beta}_{s}^{(k)} \mathbf{w}_{s}$;

31. $\quad \mathbf{g}_{k}=\mathbf{g}_{k}+\tilde{\beta}_{s}^{(k)} \mathbf{g}_{s}$;

32. End

33. $\mathbf{g}_{k}=\mathbf{z}_{w}+\frac{1}{\rho_{g_{n}(k+1)}} \mathbf{g}_{k}$;

34. $\quad \mathbf{w}_{k}=\mathbf{A g}_{k}$;

35. End

36. $\quad c_{k}=\mathbf{q}_{r_{n}(k+1)}^{H} \mathbf{w}_{k}$;

\section{3\%. End}

We remark that (i) the algorithm does not compute $\mathbf{u}_{k}$ when $r_{n}(k)<n$. In fact, $\mathbf{u}_{k}=\mathbf{r}_{k}$ when $r_{n}(k)<n$ (see the remark right after (5.2)); (ii) if the $\mathbf{u}_{k}$ on Line 23 happens to be zero, then the $\mathbf{x}_{k}$ on Line 22 will be the exact solution to system (3.1) and the algorithm stops there.

The cost and storage requirement, obtained from its preconditioned version, Algorithm 9.2 in 99, are listed in Table 5.1. Compared to Algorithm 4.1, Algorithm 5.1 saves about 20\% in saxpy. Since only three sets of vectors $\left\{\mathbf{q}_{1}, \ldots, \mathbf{q}_{n}\right\},\left\{\mathbf{g}_{k-n}, \ldots, \mathbf{g}_{k-1}\right\}$ and $\left\{\mathbf{w}_{k-n}, \ldots, \mathbf{w}_{k-1}\right\}$ are needed in iteration $k$, the storage is about $3 n N$ besides storing $\mathbf{A}$ and $\mathbf{M}$. 
TABLE 5.1. Average cost per ( $k$-)iteration step of Algorithm 9.2 and its storage requirement.

\begin{tabular}{|c|c|c|c|}
\hline Preconditioning $\left(\mathbf{M}^{-1} \mathbf{v}\right)$ & $1+\frac{1}{n}$ & Vector addition $(\mathbf{u} \pm \mathbf{v})$ & 1 \\
\hline Matvec $(\mathbf{A v})$ & $1+\frac{1}{n}$ & Saxpy $(\mathbf{u}+\alpha \mathbf{v})$ & $2 n+2+\frac{2}{n}$ \\
\hline $\operatorname{dot}$ product $\left(\mathbf{u}^{H} \mathbf{v}\right)$ & $n+1+\frac{2}{n}$ & Storage & $\mathbf{A}+\mathbf{M}+(3 n+5) N+O(n)$ \\
\hline
\end{tabular}

5.3. Properties. We summarize the properties about Algorithm 5.1 below. Their proofs are similar to those in Proposition 4.1. Since $\mathbf{r}_{0}=\widehat{\mathbf{r}}_{0}$ by (5.3), $\nu$ is also the degree of the minimal polynomial of $\mathbf{r}_{0}$ with respect to $\mathbf{A}$.

Proposition 5.1. Under the assumptions of Proposition [3.1, if $\rho_{g_{n}(k+1)} \neq 0$ and $-1 / \rho_{g_{n}(k+1)} \notin$ $\sigma(\mathbf{A})$ for $1 \leq k \leq \nu-1$, then Algorithm 5.1 does not break down by zero division for $k=1,2, \cdots, \nu$, and the approximate solution $\mathbf{x}_{\nu}$ at step $k=\nu$ is exact to the system (3.1). Moreover, the computed quantities satisfy

(a) $\mathbf{x}_{k} \in \mathbf{x}_{0}+\operatorname{span}\left\{\mathbf{r}_{0}, \mathbf{A} \mathbf{r}_{0}, \ldots, \mathbf{A}^{g_{n}(k+1)+k-1} \mathbf{r}_{0}\right\}$ and $\mathbf{r}_{k}=\mathbf{b}-\mathbf{A} \mathbf{x}_{k} \in \mathbf{r}_{0}+\operatorname{span}\left\{\mathbf{A r}_{0}, \mathbf{A}^{2} \mathbf{r}_{0}, \ldots, \mathbf{A}^{g_{n}(k+1)+k} \mathbf{r}_{0}\right\}$ for $1 \leq k \leq \nu-1$.

(b) $\mathbf{r}_{k} \neq \mathbf{0}$ for $1 \leq k \leq \nu-1 ; \mathbf{r}_{\nu}=\mathbf{0}$.

(c) $\mathbf{r}_{k} \perp \operatorname{span}\left\{\mathbf{q}_{1}, \mathbf{q}_{2}, \cdots, \mathbf{q}_{r_{n}(k)}\right\}$ and $\mathbf{r}_{k} \not \perp \mathbf{q}_{r_{n}(k)+1}$ for $1 \leq k \leq \nu-1$ with $r_{n}(k)<n ; \mathbf{r}_{k} \not \perp \mathbf{q}_{1}$ for $1 \leq k \leq \nu-1$ with $r_{n}(k)=n$.

(d) $\mathbf{u}_{k} \perp \operatorname{span}\left\{\mathbf{q}_{1}, \mathbf{q}_{2}, \cdots, \mathbf{q}_{n}\right\}$ for $1 \leq k \leq \nu$ with $r_{n}(k)=n$.

(e) $\mathbf{A g}_{k} \perp \operatorname{span}\left\{\mathbf{q}_{1}, \mathbf{q}_{2}, \cdots, \mathbf{q}_{r_{n}(k)}\right\}$ and $\mathbf{A g}_{k} \not \perp \mathbf{q}_{r_{n}(k)+1}$ for $1 \leq k \leq \nu-1$ with $r_{n}(k)<n$; $\operatorname{Ag}_{k} \not \perp \mathbf{q}_{1}$ for $1 \leq k \leq \nu-1$ with $r_{n}(k)=n$.

\section{Relations to Some Other Methods}

In this section, we discuss the relations of $\mathrm{ML}(n)$ BiCGStab with the FOM, BiCGStab and $\operatorname{IDR}(n)$ methods under the exact arithmetic environment.

\subsection{Algorithm 4.1.}

(1) Relation with FOM [22]. Consider the case where $n \geq \nu$. In this case, $g_{n}(k)=0$ and $r_{n}(k)=k$ for $k=1,2, \cdots, \nu$. Hence $\mathbf{p}_{k}=\mathbf{q}_{k}$ by (3.2). If we choose $\mathbf{q}_{k}=\widehat{\mathbf{r}}_{k-1}$ in Algorithm 3.1 (it is possible since $\widehat{\mathbf{r}}_{k-1}$ is computed before $\mathbf{q}_{k}$ is used), then the $\widehat{\mathbf{x}}_{k}$ and $\widehat{\mathbf{r}}_{k}$ computed by the algorithm satisfy

$$
\left\{\begin{array}{l}
\widehat{\mathbf{x}}_{k} \in \widehat{\mathbf{x}}_{0}+\operatorname{span}\left\{\widehat{\mathbf{r}}_{0}, \mathbf{A} \widehat{\mathbf{r}}_{0}, \ldots, \mathbf{A}^{k-1} \widehat{\mathbf{r}}_{0}\right\}, \\
\widehat{\mathbf{r}}_{k} \perp \operatorname{span}\left\{\widehat{\mathbf{r}}_{0}, \widehat{\mathbf{r}}_{1}, \ldots, \widehat{\mathbf{r}}_{k-1}\right\}
\end{array}\right.
$$

for $1 \leq k \leq \nu$ by Proposition 3.1(a), (d). (6.1) is what the FOM approximate solution $\mathbf{x}_{k}^{F O M}$ needs to satisfy. Therefore, when $n \geq \nu$ and with the choice $\mathbf{q}_{k}=\widehat{\mathbf{r}}_{k-1}$, Algorithm 3.1 is mathematically equivalent to FOM.

Now, from (4.3), the $\mathbf{r}_{k}$ computed by Algorithm 4.1 satisfies

$$
\mathbf{r}_{k}=\phi_{g_{n}(k)+1}(\mathbf{A}) \widehat{\mathbf{r}}_{k}=\phi_{1}(\mathbf{A}) \widehat{\mathbf{r}}_{k}=\left(\rho_{1} \mathbf{A}+\mathbf{I}\right) \widehat{\mathbf{r}}_{k} .
$$

Note that $\mathbf{u}_{k}=\phi_{g_{n}(k)}(\mathbf{A}) \widehat{\mathbf{r}}_{k}=\phi_{0}(\mathbf{A}) \widehat{\mathbf{r}}_{k}=\widehat{\mathbf{r}}_{k}$. Thus, for $1 \leq k \leq \nu, \mathbf{r}_{k}$ is the factor $\rho_{1} \mathbf{A}+\mathbf{I}$ times the FOM residual $\mathbf{u}_{k}$ if we set $\mathbf{q}_{1}=\mathbf{r}_{0}$ and $\mathbf{q}_{k+1}=\mathbf{u}_{k}$ in Algorithm 4.1, ${ }^{6}$

\footnotetext{
${ }^{6}$ In [39], a remark immediately following Theorem 4.1 states that, when $n \geq \nu$ and with the choice that $\mathbf{q}_{1}=$ $\phi_{1}\left(\mathbf{A}^{H}\right) \phi_{1}(\mathbf{A}) \mathbf{r}_{0}$ and $\mathbf{q}_{k}=\phi_{1}\left(\mathbf{A}^{H}\right) \mathbf{r}_{k-1}$ for $k \geq 2$, the $\mathbf{x}_{k}$ and $\mathbf{r}_{k}$ computed by Algorithm 2 (which is mathematically
} 
(2) Relation with BiCGStab [32]. When $n=1$, we have $g_{n}(k)=k-1$ and $r_{n}(k)=1$ for $k \in \mathcal{N}$. Hence $\mathbf{p}_{k}=\left(\mathbf{A}^{H}\right)^{k-1} \mathbf{q}_{1}$ by (3.2). By Proposition 3.1 (a) and (d), the $\widehat{\mathbf{x}}_{k}$ and $\widehat{\mathbf{r}}_{k}$ computed by Algorithm 3.1 satisfy

$$
\left\{\begin{array}{l}
\widehat{\mathbf{x}}_{k} \in \widehat{\mathbf{x}}_{0}+\operatorname{span}\left\{\widehat{\mathbf{r}}_{0}, \mathbf{A} \widehat{\mathbf{r}}_{0}, \ldots, \mathbf{A}^{k-1} \widehat{\mathbf{r}}_{0}\right\} \\
\widehat{\mathbf{r}}_{k} \perp \operatorname{span}\left\{\mathbf{q}_{1}, \mathbf{A}^{H} \mathbf{q}_{1}, \ldots,\left(\mathbf{A}^{H}\right)^{k-1} \mathbf{q}_{1}\right\}
\end{array}\right.
$$

for $1 \leq k \leq \nu$. (6.2) is what the BiCG approximate solution $\mathbf{x}_{k}^{B i C G}$ needs to satisfy. Therefore, when $n=1$, Algorithm 3.1 is mathematically equivalent to BiCG.

Now, from (4.3), the $\mathbf{r}_{k}$ computed by Algorithm 4.1 satisfies

$$
\mathbf{r}_{k}=\phi_{g_{n}(k)+1}(\mathbf{A}) \widehat{\mathbf{r}}_{k}=\phi_{k}(\mathbf{A}) \widehat{\mathbf{r}}_{k}
$$

which is the definition of the BiCGStab residuals. Thus Algorithm 4.1 is mathematically equivalent to BiCGStab when $n=1$.

(3) Relation with $\operatorname{IDR}(n)$ [31]. Write $k=j n+i$ as in (2.1) with $1 \leq i \leq n, 0 \leq j$. Let

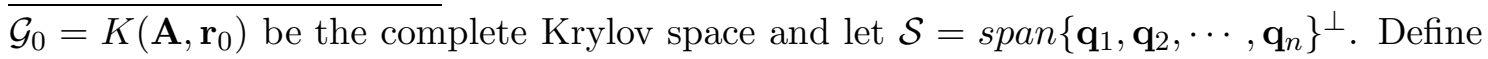
the Sonneveld spaces

$$
\mathcal{G}_{j+1}=\left(\rho_{j+1} \mathbf{A}+\mathbf{I}\right)\left(\mathcal{G}_{j} \cap \mathcal{S}\right)=\left(\rho_{g_{n}(k)+1} \mathbf{A}+\mathbf{I}\right)\left(\mathcal{G}_{j} \cap \mathcal{S}\right)
$$

for $j=0,1,2, \cdots$. By (4.3), we have

$$
\mathbf{r}_{j n+i}=\phi_{j+1}(\mathbf{A}) \widehat{\mathbf{r}}_{j n+i}=\left(\rho_{j+1} \mathbf{A}+\mathbf{I}\right) \phi_{j}(\mathbf{A}) \widehat{\mathbf{r}}_{j n+i}=\left(\rho_{j+1} \mathbf{A}+\mathbf{I}\right) \mathbf{u}_{j n+i} .
$$

From Proposition $4.1(\mathrm{~d}), \mathbf{u}_{j n+i} \not \perp \mathbf{q}_{i+1}$ if $i<n$. Hence $\mathbf{u}_{j n+i} \notin \mathcal{G}_{j} \cap \mathcal{S}$ and therefore $\mathbf{r}_{j n+i} \notin \mathcal{G}_{j+1}$ when $i<n$. From this point of view, Algorithm 4.1 is not a $\operatorname{IDR}(n)$ algorithm.

\subsection{Algorithm 5.1.}

(1) Relation with FOM. When $n \geq \nu, g_{n}(k)=0$ and $r_{n}(k)=k$ for $1 \leq k \leq \nu$ and Algorithm 3.1, with the choice $\mathbf{q}_{k}=\widehat{\mathbf{r}}_{k-1}$, is a FOM algorithm as seen in 6.1 , Now, from (5.2), the $\mathbf{r}_{k}$ computed by Algorithm [5.1 satisfies

$$
\mathbf{r}_{k}=\phi_{g_{n}(k+1)}(\mathbf{A}) \widehat{\mathbf{r}}_{k}=\phi_{0}(\mathbf{A}) \widehat{\mathbf{r}}_{k}=\widehat{\mathbf{r}}_{k} .
$$

Thus Algorithm 5.1 is a FOM algorithm when we set $\mathbf{q}_{k}=\mathbf{r}_{k-1}$.

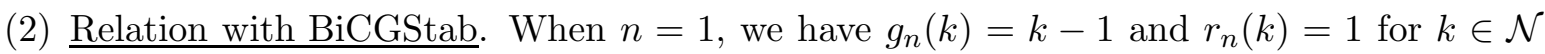
and Algorithm 3.1 is a BiCG algorithm. Now, from (5.2), the $\mathbf{r}_{k}$ computed by Algorithm 5.1 satisfies

$$
\mathbf{r}_{k}=\phi_{g_{n}(k+1)}(\mathbf{A}) \widehat{\mathbf{r}}_{k}=\phi_{k}(\mathbf{A}) \widehat{\mathbf{r}}_{k}
$$

which is the definition of the BiCGStab residuals. Thus Algorithm 5.1 is mathematically equivalent to BiCGStab.

(3) Relation with $\operatorname{IDR}(n)$. Write $k=j n+i$ as in (2.1) with $1 \leq i \leq n, 0 \leq j$. By (5.2), we have

$$
\mathbf{r}_{j n+i}=\phi_{g_{n}(j n+i+1)}(\mathbf{A}) \widehat{\mathbf{r}}_{j n+i}= \begin{cases}\phi_{j}(\mathbf{A}) \widehat{\mathbf{r}}_{j n+i} & \text { if } 1 \leq i<n \\ \phi_{j+1}(\mathbf{A}) \widehat{\mathbf{r}}_{j n+i} & \text { if } i=n\end{cases}
$$

equivalent to Algorithm 4.1 of this paper) will satisfies (6.1) and therefore Algorithm 2 is a FOM. The argument there about this remark is not correct. The author remembers that the referees of [39] were skeptical about the argument. 
By (3.2) and Proposition 3.1(d), we have

$$
\begin{cases}\phi_{t}(\mathbf{A}) \widehat{\mathbf{r}}_{j n+i} \in \mathcal{S} & \text { if } 1 \leq i<n \text { and } 0 \leq t<j \\ \phi_{t}(\mathbf{A}) \widehat{\mathbf{r}}_{j n+n} \in \mathcal{S} & \text { if } 0 \leq t \leq j\end{cases}
$$

Thus, by induction on $t$, we have

$$
\begin{cases}\phi_{t}(\mathbf{A}) \widehat{\mathbf{r}}_{j n+i} \in \mathcal{G}_{t} \cap \mathcal{S} & \text { if } 1 \leq i<n \text { and } 0 \leq t<j \\ \phi_{t}(\mathbf{A}) \widehat{\mathbf{r}}_{j n+n} \in \mathcal{G}_{t} \cap \mathcal{S} & \text { if } 0 \leq t \leq j .\end{cases}
$$

Therefore, by (6.3),

$$
\begin{cases}\mathbf{r}_{j n+i}=\widehat{\mathbf{r}}_{i} \in \mathcal{G}_{0} & \text { if } 1 \leq i<n, j=0, \\ \mathbf{r}_{j n+i}=\left(\rho_{j} \mathbf{A}+\mathbf{I}\right) \phi_{j-1}(\mathbf{A}) \widehat{\mathbf{r}}_{j n+i} \in \mathcal{G}_{j} & \text { if } 1 \leq i<n, 1 \leq j, \\ \mathbf{r}_{j n+n}=\left(\rho_{j+1} \mathbf{A}+\mathbf{I}\right) \phi_{j}(\mathbf{A}) \widehat{\mathbf{r}}_{j n+n} \in \mathcal{G}_{j+1} . & \end{cases}
$$

So, the residuals in (6.3) lie in the Sonneveld spaces $\mathcal{G}$ and therefore Algorithm 5.1 is a $\operatorname{IDR}(n)$ algortithm.

\section{IMPLEMENTATION ISSUES}

A preconditioned $\operatorname{ML}(n)$ BiCGStab algorithm can be obtained by applying either Algorithm 4.1 or Algorithm 5.1 to the system

$$
\mathrm{AM}^{-1} \mathbf{y}=\mathbf{b}
$$

where $\mathbf{M}$ is nonsingular, then recovering $\mathbf{x}$ through $\mathbf{x}=\mathbf{M}^{-1} \mathbf{y}$. The resulting algorithms, Algorithm 9.1 and Algorithm 9.2, together with their Matlab codes are presented in 99.1 and 99.2 respectively. To avoid calling the index functions $r_{n}(k)$ and $g_{n}(k)$ every $k$-iteration, we have split the $k$-loop into a $i$-loop and a $j$-loop where $i, j, k$ are related by (2.1) with $1 \leq i \leq n, 0 \leq j$. Moreover, we have optimized the operations as possible as we can in the resulting preconditioned algorithms.

Since we have compared ML( $n)$ BiCGStab with some existing methods in [39], we will only concentrate on the performance of $\mathrm{ML}(n)$ BiCGStab itself. The following test data were downloaded from Matrix Market. ${ }^{7}$

(1) e20r0100, DRIVCAV Fluid Dynamics. e20r0100 contains a $4241 \times 4241$ real unsymmetric matrix $\mathbf{A}$ with 131, 556 nonzero entries and a real right-hand side $\mathbf{b}$.

(2) qc2534, H2PLUS Quantum Chemistry, NEP Collection. qc2534 contains a $2534 \times 2534$ complex symmetric indefinite matrix with 463,360 nonzero entries, but does not provide the right-hand side $\mathbf{b}$. Following [25, we set $\mathbf{b}=\mathbf{A} \mathbf{1}$ with $\mathbf{1}=[1,1, \cdots, 1]^{T}$.

(3) utm5940, TOKAMAK Nuclear Physics (Plasmas). utm5940 contains a $5940 \times 5940$ real unsymmetric matrix $\mathbf{A}$ with 83, 842 nonzero entries and a real right-hand side $\mathbf{b}$.

Experiments were performed in Matlab Version 7.1 on a Windows XP machine with a Pentium 4 processor. $I L U(0)$ preconditioner (p.294, [21]) has been used in all the experiments. For e20r0100, the $U$-factor of the $I L U(0)$ decomposition of $\mathbf{A}$ has some zeros along its main diagonal. In that experiment, we replaced those zeros with 1 so that the $U$-factor was invertible.

In all the experiments, initial guess $\mathbf{x}_{0}=\mathbf{0}$ and stopping criterion is

$$
\left\|\mathbf{r}_{k}\right\|_{2} /\|\mathbf{b}\|_{2}<10^{-7}
$$

where $\mathbf{r}_{k}$ is the computed residual. Except where specified, auxiliary vectors $\mathbf{Q} \equiv\left[\mathbf{q}_{1}, \mathbf{q}_{2}, \cdots, \mathbf{q}_{n}\right]$ are chosen to be $\mathbf{Q}=\left[\mathbf{r}_{0}, \operatorname{randn}(N, n-1)\right]$ for e20r0100 and utm5940 and $\mathbf{Q}=\left[\mathbf{r}_{0}, \operatorname{randn}(N, n-\right.$ $1)+\operatorname{sqrt}(-1) * \operatorname{randn}(N, n-1)]$ for $q$ c2534.

\footnotetext{
${ }^{7}$ http://math.nist.gov/MatrixMarket/data/
} 
Moreover, for the convenience of our presentation, we introduce the following functions:

(a) $T_{\text {conv }}(n)$ is the time that a $\operatorname{ML}(n)$ BiCGStab algorithm takes to converge.

(b) $I_{c o n v}(n)$ is the number of iterations that a $\mathrm{ML}(n) \operatorname{BiCGStab}$ algorithm takes to converge.

(c) $E(n):=\|\mathbf{b}-\mathbf{A} \mathbf{x}\|_{2} /\|\mathbf{b}\|_{2}$ is the true relative error of $\mathbf{x}$ where $\mathbf{x}$ is the computed solution output by a $\operatorname{ML}(n)$ BiCGStab algorithm when it converges.

7.1. Stability. We plot the graphs of $I_{\text {conv }}(n)$ in Figures 7.1(a), 7.2(a) and 7.3(a). For e20r0100 and qc2534, $I_{\text {conv }}(n)$ decreases as $n$ increases. However, the $I_{c o n v}(n)$ for utm5940 behaves very irregularly due to some of the $\rho$ 's are too small. Recall that $\operatorname{ML}(n) \operatorname{BiCGStab}$ performs $1+1 / n$ matrix-vector multiplications (MVs) per iteration on average. In terms of the number of MVs, both Algorithms 9.1 and 9.2 are considerably faster than BiCGStab in all the three experiments.
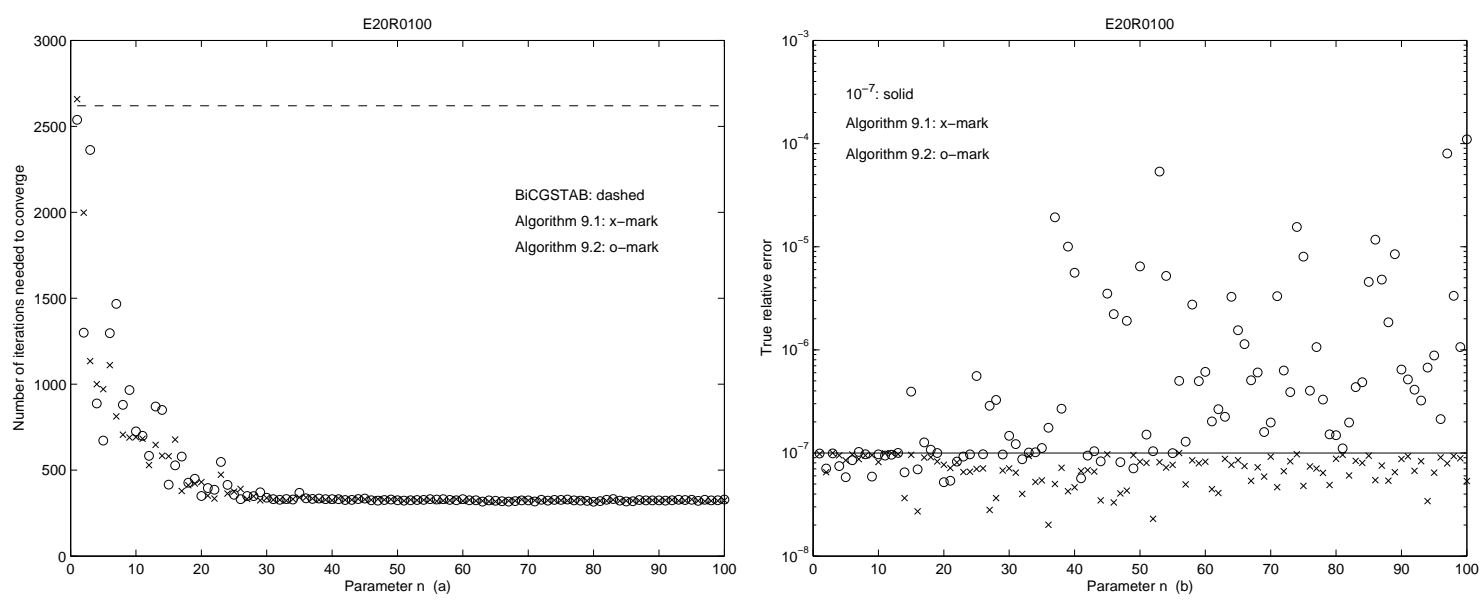

FiguRE 7.1. e20r0100: (a) Graphs of $I_{\text {conv }}(n)$ against $n$. BiCGStab took 2620 iterations/5240 MVs to converge. Full GMRES converged with 308 MVs. (b) Graphs of $E(n)$ against $n$.
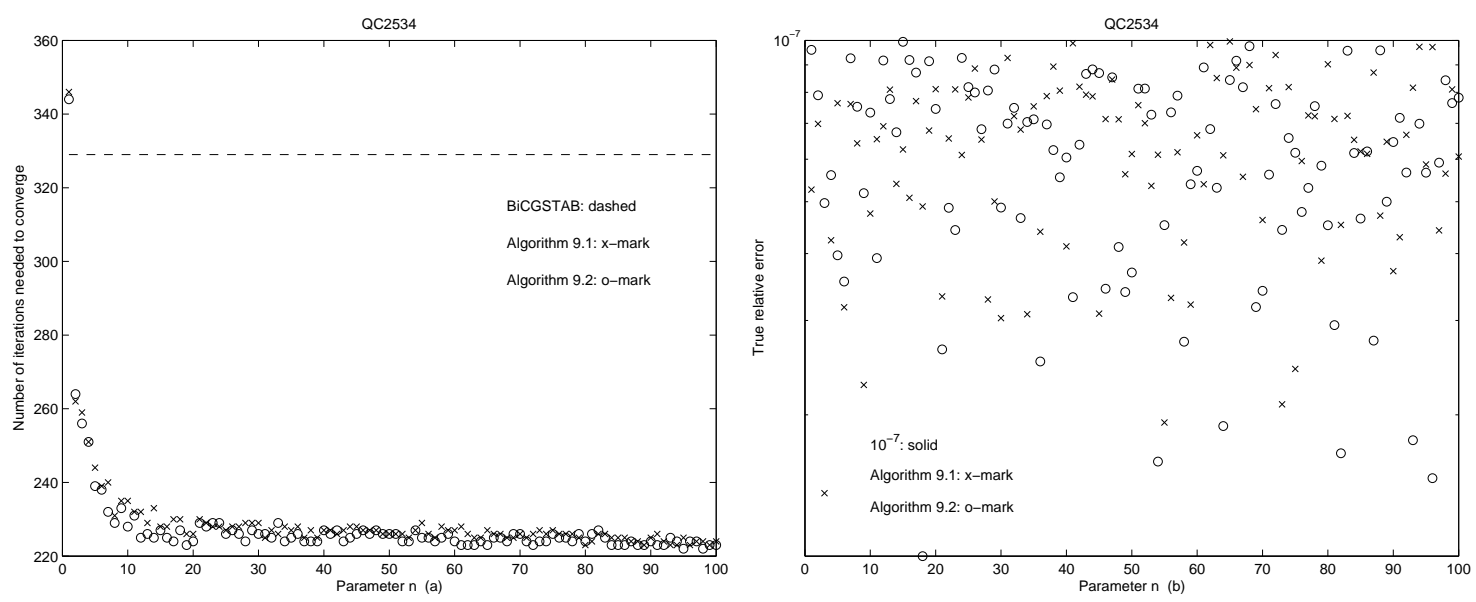

FiguRE 7.2. qc2534: (a) Graphs of $I_{\text {conv }}(n)$ against $n$. BiCGStab took 329 iterations/658 MVs to converge. Full GMRES converged with 439 MVs. (b) Graphs of $E(n)$ against $n$. 

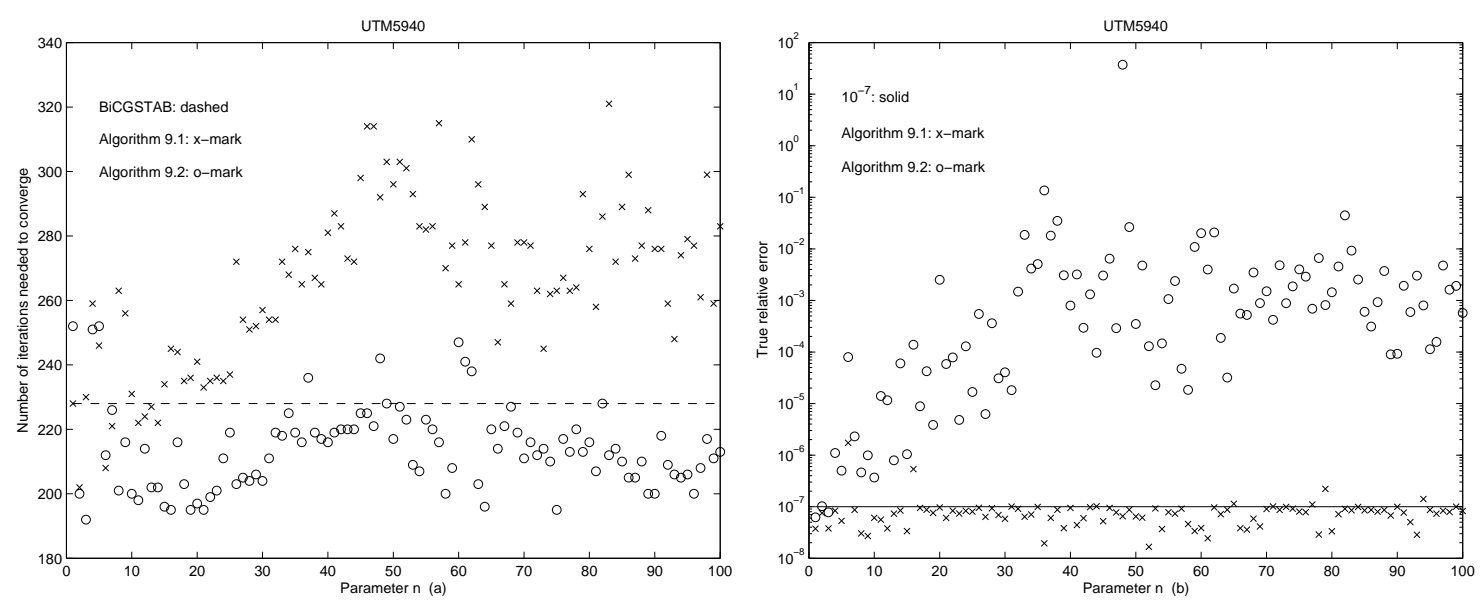

Figure 7.3. utm5940: (a) Graphs of $I_{\text {conv }}(n)$ against $n$. BiCGStab took 228 iterations/455 MVs to converge. Full GMRES converged with $176 \mathrm{MVs.}$ (b) Graphs of $E(n)$ against $n$.

The graphs of $E(n)$ are plotted in Figures 7.1(b), 7.2(b) and 7.3(b). It can be seen that the computed $\mathbf{r}_{k}$ in Algorithm 9.2 easily diverges from its exact counterpart $\mathbf{b}-\mathbf{A} \mathbf{x}_{k}$. This divergence becomes significant when $n \geq 15$ for e20r0100 and $n \geq 4$ for utm5940. By contrast, the computed relative errors $\left\|\mathbf{r}_{k}\right\|_{2} /\|\mathbf{b}\|_{2}$ by Algorithm 9.1 well approximate their corresponding true ones. Thus, from this point of view, we consider that Algorithm 9.1 is numerically more stable than Algorithm 9.2. However, Algorithm 9.2 taken twice to form a predictor-corrector pair can be an efficient and stable algorithm. We remark that the issues of divergence of computed residuals and corresponding remedy techniques have been discussed in detail in [18, 27, 34].

7.2. Choice of $n$. In this and the following subsections, we will focus on Algorithm 9.1,

From the experiments in [39] and this paper, we have observed that $\mathrm{ML}(n) \operatorname{BiCGStab}$ behaves more and more robust as $n$ is increased. So, for an ill-conditioned problem, we would tend to suggest a large $n$ for $\operatorname{ML}(n) \operatorname{BiCGStab.~}$

$\operatorname{ML}(n)$ BiCGStab minimizes $\left\|\mathbf{r}_{k}\right\|_{2}$ once every $n$ iterations. The convergence of a well-conditioned problem is usually accelerated by the minimization steps. So, when a problem is well-conditioned, we would suggest a small $n$, say, $n \leq 3$. $n=2$ may be a good choice since it reduces the MV cost by $25 \%$ per iteration while keeping the minimization performed with a high frequency.

We also plot the graphs of $T_{\text {conv }}(n)$ in Figures 7.4 and 7.5(a) to provide more information on how $n$ affects the performance of $\operatorname{ML}(n) \operatorname{BiCGStab.}$

7.3. Choice of $\rho$. The standard choice for the $\rho_{j+1}$ in Algorithm 9.1 is

$$
\rho_{j+1}=-\left(\mathbf{A} \widetilde{\mathbf{u}}_{j n+1}\right)^{H} \mathbf{u}_{j n+1} /\left\|\mathbf{A} \widetilde{\mathbf{u}}_{j n+1}\right\|_{2}^{2} .
$$

This choice of $\rho_{j+1}$ minimizes the 2-norm of $\mathbf{r}_{j n+1}=\rho_{j+1} \mathbf{A} \widetilde{\mathbf{u}}_{j n+1}+\mathbf{u}_{j n+1}$, but sometimes can cause instability due to that it can be very small during an implementation. A remedy as follows has been suggested in [26]:

$$
\begin{aligned}
& \rho_{j+1}=-\left(\mathbf{A} \widetilde{\mathbf{u}}_{j n+1}\right)^{H} \mathbf{u}_{j n+1} /\left\|\mathbf{A} \widetilde{\mathbf{u}}_{j n+1}\right\|_{2}^{2} \\
& \omega=\left(\mathbf{A} \widetilde{\mathbf{u}}_{j n+1}\right)^{H} \mathbf{u}_{j n+1} /\left(\left\|\mathbf{A} \widetilde{\mathbf{u}}_{j n+1}\right\|_{2}\left\|\mathbf{u}_{j n+1}\right\|_{2}\right) ; \\
& \text { if }|\omega|<\kappa, \quad \rho_{j+1}=\kappa \rho_{j+1} /|\omega| ; \text { end }
\end{aligned}
$$

where $\kappa$ is a user-defined parameter. In Figures 7.5(b) and 7.6(a), we compare the performances of Algorithm 9.1 with (7.1) and (7.2) respectively (we only plot the results of qc2534 and utm5940. 

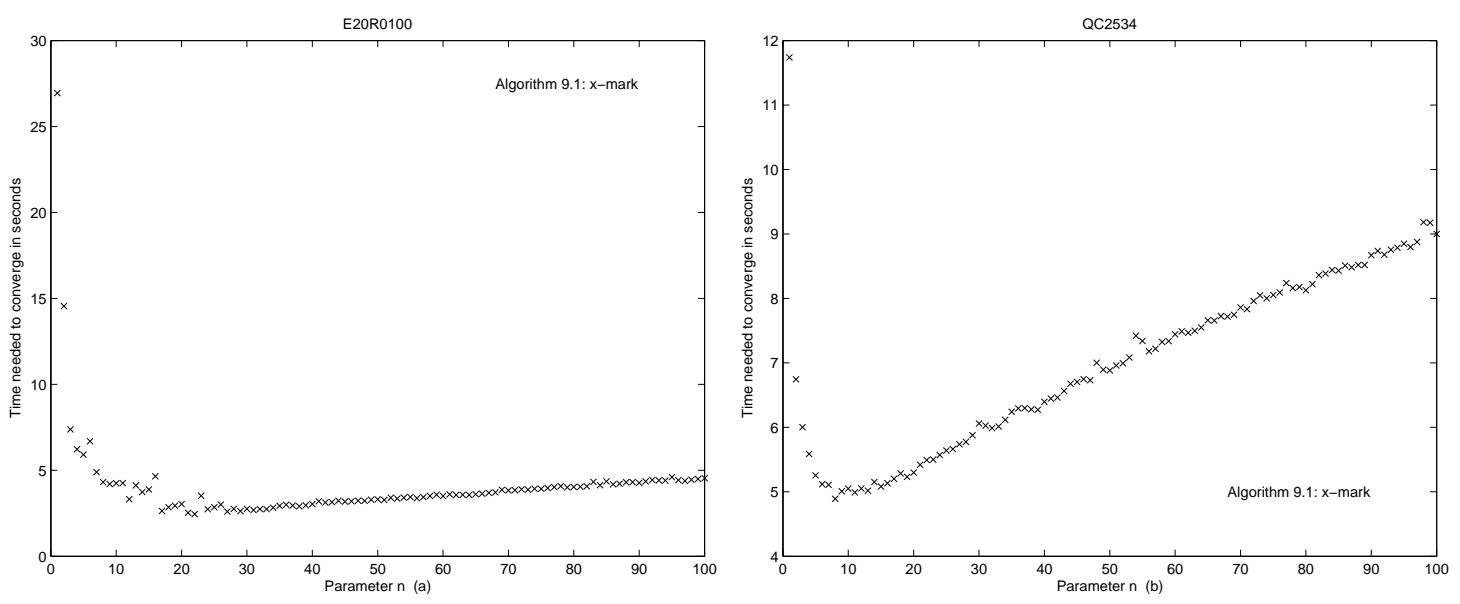

Figure 7.4. (a) e20r0100: Graph of $T_{\text {conv }}(n)$ of Algorithm 9.1 against $n$. $T_{\text {conv }}(n)$ reaches its minimum at $n=22$. (b) qc2534: Graph of $T_{\text {conv }}(n)$ of Algorithm 9.1 against $n . T_{\text {conv }}(n)$ reaches its minimum at $n=8$.
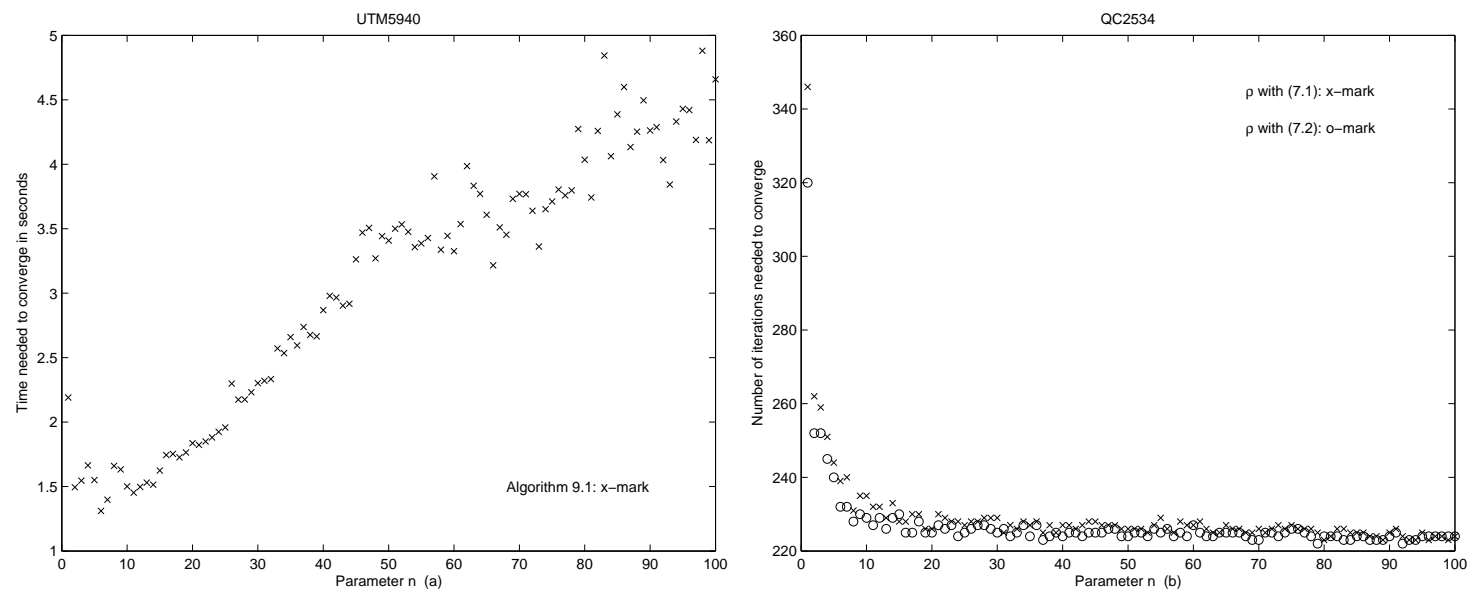

FigurE 7.5. (a) utm5940: Graph of $T_{\text {conv }}(n)$ of Algorithm 9.1 against $n$. $T_{\text {conv }}(n)$ reaches its minimum at $n=6$. (b) qc2534: Graphs of $I_{c o n v}(n)$ of Algorithm 9.1 against $n$ with choices (7.1) and (7.2) for $\rho$ respectively. In this experiment, we picked $\kappa=0.7$.

The result of e20r0100 with $\kappa=0.1$ is analogous to Figure 7.5(b)). Also, see the numerical experiments in [31] for more information about these $\rho$ choices.

7.4. Choice of $\mathbf{q}$ 's. We usually pick $\mathbf{Q}=\left[\mathbf{q}_{1}, \mathbf{q}_{2}, \cdots, \mathbf{q}_{n}\right]$ as

$$
\mathbf{Q}=\left[\mathbf{r}_{0}, \operatorname{rand} n(N, n-1)\right]
$$

for a real problem and

$$
\mathbf{Q}=\left[\mathbf{r}_{0}, \operatorname{randn}(N, n-1)+\operatorname{sqrt}(-1) * \operatorname{randn}(N, n-1)\right]
$$

for a complex problem. In our experiments, however, we observed a comparable performance when we chose

$$
\mathbf{Q}=\left[\mathbf{r}_{0}, \operatorname{sign}(\operatorname{randn}(N, n-1))\right]
$$



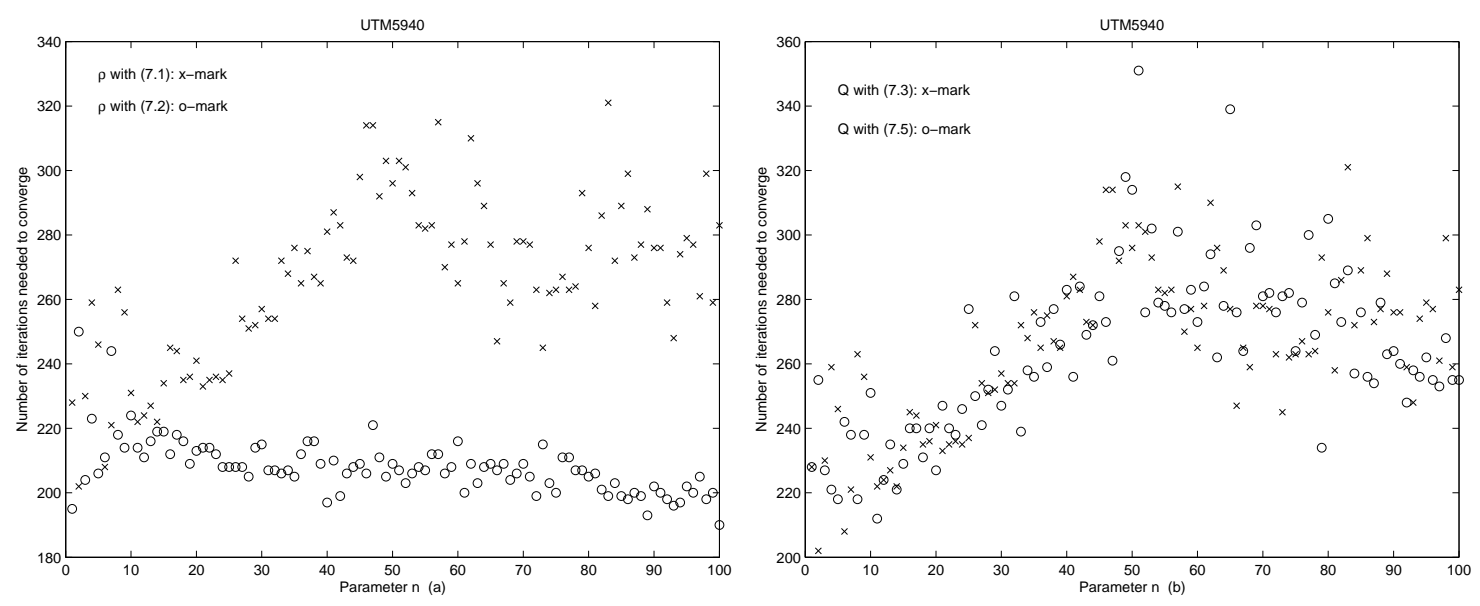

Figure 7.6. utm5940: (a) Graphs of $I_{\text {conv }}(n)$ of Algorithm 9.1 against $n$ with choices (7.1) and (7.2) for $\rho$ respectively. In this experiment, we picked $\kappa=0.7$. (b) Graphs of $I_{\text {conv }}(n)$ of Algorithm 9.1 against $n$ with choices (7.3) and (17.5) for $\mathbf{Q}$ respectively.

or

$$
\mathbf{Q}=\left[\mathbf{r}_{0}, \operatorname{sign}(\operatorname{randn}(N, n-1))+\operatorname{sqrt}(-1) * \operatorname{sign}(\operatorname{randn}(N, n-1))\right]
$$

See Figure 7.6(b) (we only plot the result of utm5940 for saving space).

The advantages of (7.5) and (7.6) over (7.3) and (7.4) are that (i) the storage of $\mathbf{Q}$ is substantially reduced. In fact, we just need to store the random signs (except its first column); (ii) an inner product with $\mathbf{q}_{i}, 2 \leq i \leq n$, is now reduced to a sum without involving scalar multiplications.

For other choices for $\mathbf{Q}$, one is referred to [31].

\section{Concluding Remarks}

With the help of index functions, we re-derived the ML(n)BiCGStab algorithm in [39] in a more systematic way. This time, we have been able to find out and remove some redundant operations so that the algorithm becomes more efficient. We also realized that there are $n$ ways to define the $\operatorname{ML}(n)$ BiCGStab residuals $\mathbf{r}_{k}$. Each of the definitions will lead to a different algorithm. We presented two definitions together with their associated algorithms, namely, (i) definition (4.3), increasing the degree of $\phi$ at the beginning of an iteration cycle, and the associated Algorithm 4.1; (ii) definition (5.2), increasing the degree of $\phi$ at the end of an iteration cycle, and the associated Algorithm 5.1. By comparison, Algorithm 5.1 is cheaper in storage and in computational cost, faster to converge, but less stable. For other definitions of $\mathbf{r}_{k}$ that increase the degree of $\phi$ somewhere within a cycle, we expect that the associated algorithms would lie between Algorithms 4.1 and 5.1 in terms of computational cost, storage and performance.

We proved that the Lanczos-based BiCG/BiCGStab and the Arnoldi-based FOM are essentially methods of the same type. Both are the extreme cases of $\mathrm{ML}(n) \operatorname{BiCG} / \mathrm{ML}(n) \operatorname{BiCGStab}$.

In this paper, we did not assume that $\mathbf{A}$ is a nonsingular matrix. When a singular system (3.1) is solved, selecting an appropriate initial guess $\widehat{\mathbf{x}}_{0}$ is a crucial step. If $\widehat{\mathbf{x}}_{0}$ is selected such that the affine space $\widehat{\mathbf{x}}_{0}+\operatorname{span}\left\{\mathbf{A}^{t} \widehat{\mathbf{r}}_{0} \mid t \in \mathcal{N}_{0}\right\}$ contains a solution to the system (3.1) ${ }^{8}, \operatorname{ML}(n) \operatorname{BiCG}$ will almost surely converge (see Corollary [3.2). Otherwise, we shall have $p_{\min }\left(0, \mathbf{A}, \widehat{\mathbf{r}}_{0}\right)=0$ (see the remark before Corollary [3.2) which yields $\operatorname{det}\left(\widehat{\mathbf{S}}_{\nu}\right)=0$ (see the remark after Proposition 3.1). In

\footnotetext{
${ }^{8}$ For an example where the affine space contains no solution, consider $\mathbf{A}=[0,1 ; 0,0], \mathbf{b}=[1,0]^{T}$ and select $\widehat{\mathbf{x}}_{0}=[1,0]^{T}$. Note that this linear system is consistent.
} 
this case, $\prod_{l=1}^{\nu} \operatorname{det}\left(\widehat{\mathbf{S}}_{l}\right)=0$ and therefore there is no guarantee that the $L U$-factorizations in the construction of $\mathrm{ML}(n) \mathrm{BiCG}$ exist (see the remark after Proposition 3.11). As a result, it is likely that $\left\|\widehat{\mathbf{r}}_{k}\right\|_{2}$ blows up to $\infty$. A similar remark also applies to $\operatorname{ML}(n) \operatorname{BiCGStab.}$

\section{APPENDIX}

In this section, we present the preconditioned $\mathrm{ML}(n) \operatorname{BiCGStab}$ algorithms together with their Matlab codes.

9.1. ML $(n)$ BiCGStab with Definition (4.3). The following algorithm is a preconditioned version of Algorithm 4.1.

\section{Algorithm 9.1. ML(n)BiCGStab with preconditioning associated with (4.3).}

1. Choose an initial guess $\mathbf{x}_{0}$ and $n$ vectors $\mathbf{q}_{1}, \mathbf{q}_{2}, \cdots, \mathbf{q}_{n}$.

2. Compute $\mathbf{r}_{0}=\mathbf{b}-\mathbf{A} \mathbf{x}_{0}$ and set $\mathbf{g}_{0}=\mathbf{r}_{0}$.

Compute $\widetilde{\mathbf{g}}_{0}=\mathbf{M}^{-1} \mathbf{g}_{0}, \mathbf{w}_{0}=\mathbf{A} \widetilde{\mathbf{g}}_{0}, c_{0}=\mathbf{q}_{1}^{H} \mathbf{w}_{0}$ and $e_{0}=\mathbf{q}_{1}^{H} \mathbf{r}_{0}$.

3. For $j=0,1,2, \cdots$

4. $\alpha_{j n+1}=e_{(j-1) n+n} / c_{(j-1) n+n}$;

5. $\quad \mathbf{u}_{j n+1}=\mathbf{r}_{(j-1) n+n}-\alpha_{j n+1} \mathbf{w}_{(j-1) n+n} ;$

6. $\quad \mathbf{x}_{j n+1}=\mathbf{x}_{(j-1) n+n}+\alpha_{j n+1} \widetilde{\mathbf{g}}_{(j-1) n+n} ;$

7. $\widetilde{\mathbf{u}}_{j n+1}=\mathbf{M}^{-1} \mathbf{u}_{j n+1}$;

8. $\rho_{j+1}=-\left(\mathbf{A} \widetilde{\mathbf{u}}_{j n+1}\right)^{H} \mathbf{u}_{j n+1} /\left\|\mathbf{A} \widetilde{\mathbf{u}}_{j n+1}\right\|_{2}^{2}$;

9. $\quad \mathbf{x}_{j n+1}=\mathbf{x}_{j n+1}-\rho_{j+1} \widetilde{\mathbf{u}}_{j n+1}$;

10. $\mathbf{r}_{j n+1}=\rho_{j+1} \mathbf{A} \widetilde{\mathbf{u}}_{j n+1}+\mathbf{u}_{j n+1}$;

11. For $i=1,2, \cdots, n-1$

12. $f_{j n+i}=\mathbf{q}_{i+1}^{H} \mathbf{u}_{j n+i}$;

13. If $j \geq 1$

14. $\quad \beta_{(j-1) n+i}^{(j n+i)}=-f_{j n+i} / c_{(j-1) n+i}$;

15. If $i \leq n-2$

16.

17. $\quad \mathbf{g}_{j n+i}=\beta_{(j-1) n+i}^{(j n+i)} \mathbf{g}_{(j-1) n+i}$;

$$
\mathbf{z}_{d}=\mathbf{u}_{j n+i}+\beta_{(j-1) n+i}^{(j n+i)} \mathbf{d}_{(j-1) n+i} ;
$$

18.

$\mathbf{z}_{w}=\beta_{(j-1) n+i}^{(j n+i)} \mathbf{w}_{(j-1) n+i} ;$

19.

20.

21.

22.

23.

24.

25.

$\beta_{(j-1) n+i+1}^{(j n+i)}=-\mathbf{q}_{i+2}^{H} \mathbf{z}_{d} / c_{(j-1) n+i+1}$;

26.

For $s=i+1, \cdots, n-2$

27.

28.

29.

$$
\begin{aligned}
& \mathbf{z}_{d}=\mathbf{z}_{d}+\beta_{(j-1) n+s}^{(j n+i)} \mathbf{d}_{(j-1) n+s} ; \\
& \mathbf{g}_{j n+i}=\mathbf{g}_{j n+i}+\beta_{(j-1) n+s}^{(j n+i)} \mathbf{g}_{(j-1) n+s} ;
\end{aligned}
$$

3.

.

$$
\mathbf{z}_{w}=\mathbf{z}_{w}+\beta_{(j-1) n+s}^{(j n+i)} \mathbf{w}_{(j-1) n+s}
$$

End

$$
\beta_{(j-1) n+s+1}^{(j n+i)}=-\mathbf{q}_{s+2}^{H} \mathbf{z}_{d} / c_{(j-1) n+s+1} \text {; }
$$

30.

$$
\mathbf{g}_{j n+i}=\mathbf{g}_{j n+i}+\beta_{(j-1) n+n-1}^{(j n+i)} \mathbf{g}_{(j-1) n+n-1} ;
$$$$
\mathbf{z}_{w}=\mathbf{z}_{w}+\beta_{(j-1) n+n-1}^{(j n+i)} \mathbf{w}_{(j-1) n+n-1} ;
$$

$$
\mathbf{z}_{w}=\mathbf{r}_{j n+i}+\rho_{j+1} \mathbf{z}_{w}
$$

$$
\text { Else }
$$

$$
\mathbf{g}_{j n+i}=\beta_{(j-1) n+n-1}^{(j n+i)} \mathbf{g}_{(j-1) n+n-1} ;
$$


31.

32.

33.

34.

35.

36.

$3 \%$.

38.

39.

40.

41.

42.

43.

44.

45.

46.

47.

48.

49.

50 .

51.

52.

53.

54.

55.

56 .

$5 \%$.

58.

59.

60. $\quad e_{j n+n}=\mathbf{q}_{1}^{H} \mathbf{r}_{j n+n}$;

61. $\tilde{\beta}_{(j-1) n+n}^{(j n+n)}=-e_{j n+n} / c_{(j-1) n+n} ; \quad \% \tilde{\beta}_{(j-1) n+n}^{(j n+n)}=\rho_{j+1} \beta_{(j-1) n+n}^{(j n+n)}$

62. $\mathbf{z}_{w}=\mathbf{r}_{j n+n}+\tilde{\beta}_{(j-1) n+n}^{(j n+n)} \mathbf{w}_{(j-1) n+n}$;

63. $\mathbf{g}_{j n+n}=\mathbf{z}_{w}+\left(\tilde{\beta}_{(j-1) n+n}^{(j n+n)} / \rho_{j+1}\right) \mathbf{g}_{(j-1) n+n}$;

64. If $n \geq 2$

65. $\quad \beta_{j n+1}^{(j n+n)}=-\mathbf{q}_{2}^{H} \mathbf{z}_{w} / c_{j n+1}$;

66. For $s=1, \cdots, n-2$

67.

68.

69.

70.

71.

72.

73.

End

$$
\mathbf{z}_{w}=\mathbf{r}_{j n+i}+\rho_{j+1} \beta_{(j-1) n+n-1}^{(j n+i)} \mathbf{w}_{(j-1) n+n-1} ;
$$

$$
\tilde{\beta}_{(j-1) n+n}^{(j n+i)}=-\mathbf{q}_{1}^{H} \mathbf{z}_{w} / c_{(j-1) n+n} ; \quad \% \tilde{\beta}_{(j-1) n+n}^{(j n+i)}=\rho_{j+1} \beta_{(j-1) n+n}^{(j n+i)}
$$

Else

$$
\tilde{\beta}_{(j-1) n+n}^{(j n+i)}=-\mathbf{q}_{1}^{H} \mathbf{r}_{j n+i} / c_{(j-1) n+n} ; \quad \% \tilde{\beta}_{(j-1) n+n}^{(j n+i)}=\rho_{j+1} \beta_{(j-1) n+n}^{(j n+i)}
$$$$
\mathbf{z}_{w}=\mathbf{r}_{j n+i}+\tilde{\beta}_{(j-1) n+n}^{(j n+i)} \mathbf{w}_{(j-1) n+n} ;
$$

End

$$
\mathbf{g}_{j n+i}=\mathbf{z}_{w}+\left(\tilde{\beta}_{(j-1) n+n}^{(j n+i)} / \rho_{j+1}\right) \mathbf{g}_{(j-1) n+n} ;
$$

$$
\begin{aligned}
& \beta_{j n+s}^{(j n+i)}=-\mathbf{q}_{s+1}^{H} \mathbf{z}_{w} / c_{j n+s} ; \\
& \mathbf{g}_{j n+i}=\mathbf{g}_{j n+i}+\beta_{j n+s}^{(j n+i)} \mathbf{g}_{j n+s} ;
\end{aligned}
$$$$
\text { End }
$$$$
\text { If } i<n-1
$$$$
\mathbf{d}_{j n+i}=\mathbf{z}_{w}-\mathbf{u}_{j n+i} ;
$$$$
c_{j n+i}=\mathbf{q}_{i+1}^{H} \mathbf{d}_{j n+i} \text {; }
$$$$
\widetilde{\alpha}_{j n+i+1}=f_{j n+i} / c_{j n+i} \text {; }
$$$$
\% \widetilde{\alpha}_{j n+i+1}=\alpha_{j n+i+1} / \rho_{j+1}
$$

Else

$$
c_{j n+i}=\mathbf{q}_{i+1}^{H}\left(\mathbf{z}_{w}-\mathbf{u}_{j n+i}\right) ;
$$

$$
\widetilde{\alpha}_{j n+i+1}=f_{j n+i} / c_{j n+i}
$$

$\% \widetilde{\alpha}_{j n+i+1}=\alpha_{j n+i+1} / \rho_{j+1}$$$
\mathbf{g}_{j n+i}=\mathbf{M}^{-1} \mathbf{g}_{j n+i}
$$$$
\mathbf{w}_{j n+i}=\mathbf{A} \widetilde{g}_{j n+i} \text {; }
$$$$
\mathbf{x}_{j n+i+1}=\mathbf{x}_{j n+i}+\rho_{j+1} \widetilde{\alpha}_{j n+i+1} \widetilde{\mathbf{g}}_{j n+i} ;
$$$$
\mathbf{r}_{j n+i+1}=\mathbf{r}_{j n+i}-\rho_{j+1} \widetilde{\alpha}_{j n+i+1} \mathbf{w}_{j n+i}
$$

$$
\mathbf{g}_{j n+n}=\mathbf{g}_{j n+n}+\beta_{j n+s}^{(j n+n)} \mathbf{g}_{j n+s} ;
$$$$
\mathbf{z}_{w}=\mathbf{z}_{w}+\beta_{j n+s}^{(j n+n)} \mathbf{d}_{j n+s} ;
$$$$
\beta_{j n+s+1}^{(j n+n)}=-\mathbf{q}_{s+2}^{H} \mathbf{z}_{w} / c_{j n+s+1} ;
$$

\section{End}

$$
\begin{aligned}
& \text { End } \\
& \widetilde{\mathbf{g}}_{j n+n}=\mathbf{M}^{-1} \mathbf{g}_{j n+n}
\end{aligned}
$$

$\mathbf{g}_{j n+n}=\mathbf{g}_{j n+n}+\beta_{j n+n-1}^{(j n+n)} \mathbf{g}_{j n+n-1} ;$ 
74. $\quad \mathbf{w}_{j n+n}=\mathbf{A} \widetilde{\mathbf{g}}_{j n+n} ;$

75. $c_{j n+n}=\mathbf{q}_{1}^{H} \mathbf{w}_{j n+n}$;

76. End

\section{Matlab code of Algorithm 9.1}

1. function $[x, e r r, i t e r, f l a g]=\operatorname{mlbicgstab}(A, x, b, Q, M$, max_it,tol,kappa $)$

2 .

3. \% input: $A$ : N-by-N matrix. $M: \quad$ N-by-N preconditioner matrix.

4. $\% \quad Q$ : N-by-n auxiliary matrix $\left[\mathbf{q}_{1}, \cdots, \mathbf{q}_{n}\right] . x$ : initial guess.

5. $\% \quad b$ : right hand side vector. max_it: maximum number of iterations.

6. $\%$ tol: error tolerance.

7. $\%$ kappa: (real number) minimization step controller:

8. $\% \quad k a p p a=0$, standard minimization

9. $\% \quad k a p p a>0$, Sleijpen-van der Vorst minimization

10. \%output: $x$ : solution computed. err: error norm. iter: number of iterations performed.

11. $\%$ flag: $=0$, solution found to tolerance

12. $\%=1$, no convergence given $\max \_$it iterations

13. $\%=-1$, breakdown.

14. \% storage: $D: N \times(n-2)$ matrix defined only when $n>2$.

15. \% $G, Q, W: N \times n$ matrices. $A, M: N \times N$ matrices.

16. $\% \quad x, r, g_{-} t, u, z, b: N \times 1$ matrices. $c: 1 \times n$ matrix.

17.

18. $N=\operatorname{size}(A, 2) ; n=\operatorname{size}(Q, 2)$;

19. $G=z \operatorname{eros}(N, n) ; W=z \operatorname{eros}(N, n) ; \quad \%$ initialize workspace for $\mathbf{d}, \mathbf{g}, \mathbf{w}$ and $c$

20. $\quad$ if $n>2, \quad D=z \operatorname{eros}(N, n-2)$; end

21. $\quad c=z \operatorname{eros}(1, n) ; \quad \%$ end initialization

22.

23. $\quad$ iter $=0 ; \operatorname{flag}=1 ; \operatorname{bnrm} 2=\operatorname{norm}(b)$;

24. if bnrm $2==0.0$, bnrm $2=1.0$; end

25.

26. $\quad r=b-A * x ;$ err $=\operatorname{norm}(r) /$ bnrm 2 ;

27. if err $<$ tol, flag $=0$; return, end

28.

29. $\quad G(:, n)=r ; g_{-} t=M \backslash r ; W(:, n)=A * g_{-} t$;

30. $\quad c(n)=Q(:, 1)^{\prime} * W(:, n)$;

31. if $c(n)==0$, flag $=-1$; return, end

32. $e=Q(:, 1)^{\prime} * r$;

33.

34. for $j=0: \max _{-} i t$

35. $\quad$ alpha $=e / c(n)$;

36. $\quad x=x+$ alpha $* g_{-} t$

37. $u=r-$ alpha $* W(:, n)$;

38. $\quad$ err $=\operatorname{norm}(u) /$ bnrm 2 ;

39. $\quad$ if err $<$ tol, flag $=0$; iter $=i t e r+1$; return, end

40.

41. $\quad g_{-} t=M \backslash u ; z=A * g_{-} t ; r h o=z^{\prime} * z$;

42. if $r h o==0$, flag $=-1$; return, end

43. omega $=z^{\prime} * u$;

44. if omega $==0$, flag $=-1$; return, end 
45. $\quad r h o=-$ omega/rho;

46. $\quad$ if kappa $>0$

47.

48.

omega $=$ omega $/(\operatorname{norm}(z) * \operatorname{norm}(u))$;

49.

abs_om = abs (omega);

$50 . \quad$ end

51. $\quad x=x-r h o * g_{-} t$;

52. $\quad r=r h o * z+u$;

53. $\quad$ err $=\operatorname{norm}(r) /$ bnrm 2 ;

54. $\quad$ iter $=$ iter +1 ;

55. $\quad$ if err $<$ tol, flag $=0$; return, end

56. $\quad$ if $i t e r ~>=$ max_it, return, end

57.

58. for $i=1: n-1$

59. $f=Q(:, i+1)^{\prime} * u$;

60. $\quad$ if $j>=1$

61.

62.

63.

64.

65.

66.

67.

68.

69.

70.

71.

72.

73.

74.

75 .

76.

77.

78.

79.

$$
\text { beta }=-f / c(i)
$$

if $i<=n-2$

$$
\begin{aligned}
& D(:, i)=u+\operatorname{bet} a * D(:, i) ; \\
& G(:, i)=\operatorname{beta} * G(:, i) ;
\end{aligned}
$$$$
W(:, i)=\text { beta } * W(:, i) \text {; }
$$$$
\text { beta }=-Q(:, i+2)^{\prime} * D(:, i) / c(i+1) \text {; }
$$

for $s=i+1: n-2$

$$
\begin{aligned}
& D(:, i)=D(:, i)+\text { beta } * D(:, s) \\
& G(:, i)=G(:, i)+\text { beta } * G(:, s)
\end{aligned}
$$$$
W(:, i)=W(:, i)+\operatorname{beta} * W(:, s) \text {; }
$$$$
\text { beta }=-Q(:, s+2)^{\prime} * D(:, i) / c(s+1) \text {; }
$$

end

$G(:, i)=G(:, i)+$ beta $* G(:, n-1)$;

$W(:, i)=W(:, i)+$ beta $* W(:, n-1)$;

else $W(:, i)=r+r h o * W(:, i) ;$

80.

81.

82.

83.

84.

85.

$G(:, i)=$ beta $* G(:, n-1) ;$

$W(:, i)=r+(r h o *$ beta $) * W(:, n-1) ;$

$$
\text { end }
$$

beta $=-Q(:, 1)^{\prime} * W(:, i) / c(n)$;

$W(:, i)=W(:, i)+$ beta $* W(:, n)$;

$G(:, i)=G(:, i)+W(:, i)+($ beta $/ r h o) * G(:, n) ;$

else

86.

87.

88.

89.

90.

91.

92.

93.

$$
\text { beta }=-Q(:, 1)^{\prime} * r / c(n)
$$

$W(:, i)=r+$ beta $* W(:, n)$;

$G(:, i)=W(:, i)+($ beta $/ r h o) * G(:, n) ;$

end

for $s=1: i-1$

$$
\begin{aligned}
& \text { beta }=-Q(:, s+1)^{\prime} * W(:, i) / c(s) \\
& G(:, i)=G(:, i)+\text { beta } * G(:, s) ;
\end{aligned}
$$

$W(:, i)=W(:, i)+$ beta $* D(:, s)$;

end

if $i<n-1$ 
94.

95.

96.

97.

98.

99.

100.

101.

102.

103.

104.

105.

106.

107.

108.

109 .

110.

111.

112.

113.

114.

115.

116.

117.

118.

119.

120.

121.

122 .

123.

124.

125.

126.

127.

128.

$D(:, i)=W(:, i)-u$

$c(i)=Q(:, i+1)^{\prime} * D(:, i)$;

if $c(i)==0$, flag $=-1$; return, end

alpha $=f / c(i)$;

$u=u-\operatorname{alph} a * D(:, i)$;

else

$$
c(i)=Q(:, i+1)^{\prime} *(W(:, i)-u)
$$

if $c(i)==0$, flag $=-1$; return, end

end

$$
\text { alpha }=f / c(i) \text {; }
$$

$g_{\_} t=M \backslash G(:, i) ; W(:, i)=A * g_{-} t$;

alpha $=$ rho $*$ alpha;

$x=x+$ alpha $* g_{-} t ;$

$r=r-\operatorname{alph} a * W(:, i)$

err $=\operatorname{norm}(r) /$ bnrm 2 ;

iter $=$ iter +1 ;

if err $<$ tol, flag $=0$; return, end

if iter $>=$ max_it, return, end end

$$
e=Q(:, 1)^{\prime} * r ; \text { beta }=-e / c(n) ;
$$

$W(:, n)=r+$ beta $* W(:, n)$;

$G(:, n)=W(:, n)+($ beta $/ r h o) * G(:, n)$;

if $n>=2$

beta $=-Q(:, 2)^{\prime} * W(:, n) / c(1)$;

for $s=1: n-2$

$G(:, n)=G(:, n)+$ beta $* G(:, s)$

$W(:, n)=W(:, n)+$ beta $* D(:, s)$;

beta $=-Q(:, s+2)^{\prime} * W(:, n) / c(s+1)$;

end

$G(:, n)=G(:, n)+\operatorname{beta} * G(:, n-1) ;$

end

$g_{\_} t=M \backslash G(:, n) ; W(:, n)=A * g_{-} t ;$

$c(n)=Q(:, 1)^{\prime} * W(:, n)$;

if $c(n)==0$, flag $=-1$; return, end

end

9.2. $\mathbf{M L}(n)$ BiCGStab with Definition (5.2). The following algorithm is a preconditioned version of Algorithm 5.1 .

\section{Algorithm 9.2. $\mathrm{ML}(n) \mathrm{BiCGStab}$ with preconditioning associated with (5.2)}

1. Choose an initial guess $\mathbf{x}_{0}$ and $n$ vectors $\mathbf{q}_{1}, \mathbf{q}_{2}, \cdots, \mathbf{q}_{n}$.

2. Compute $\mathbf{r}_{0}=\mathbf{b}-\mathbf{A} \mathbf{x}_{0}, \widetilde{\mathbf{g}}_{0}=\mathbf{M}^{-1} \mathbf{r}_{0}, \mathbf{w}_{0}=\mathbf{A} \widetilde{\mathbf{g}}_{0}, c_{0}=\mathbf{q}_{1}^{H} \mathbf{w}_{0}$ and $e_{0}=\mathbf{q}_{1}^{H} \mathbf{r}_{0}$.

3. For $j=0,1,2, \cdots$

4. For $i=1,2, \cdots, n-1$

5. $\quad \alpha_{j n+i}=e_{j n+i-1} / c_{j n+i-1}$

6. $\quad \mathbf{x}_{j n+i}=\mathbf{x}_{j n+i-1}+\alpha_{j n+i} \widetilde{\mathbf{g}}_{j n+i-1} ; \quad \% \widetilde{\mathbf{g}}=\mathbf{M}^{-1} \mathbf{g}$

7. $\quad \mathbf{r}_{j n+i}=\mathbf{r}_{j n+i-1}-\alpha_{j n+i} \mathbf{w}_{j n+i-1}$;

8. $\quad e_{j n+i}=\mathbf{q}_{i+1}^{H} \mathbf{r}_{j n+i}$;

9. If $j \geq 1$ 


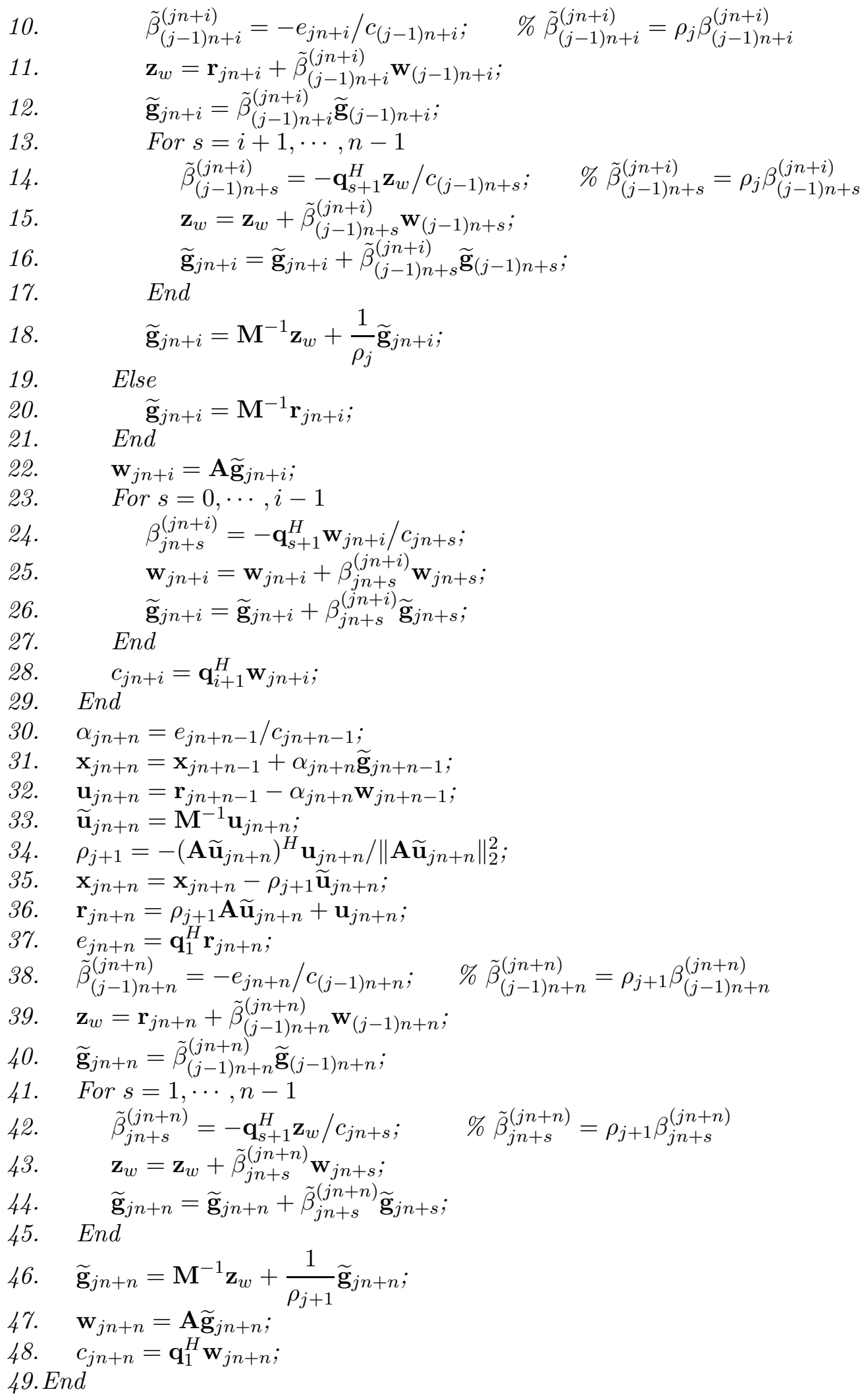

\section{Matlab code of Algorithm 9.2}


1. function $[x, e r r, i t e r, f l a g]=\operatorname{mlbicgstab}(A, x, b, Q, M$, max_it,tol,kappa $)$

2 .

3. \% input: $A$ : N-by-N matrix. $M$ : N-by-N preconditioner matrix

4. $\% Q$ : N-by-n auxiliary matrix with columns $\mathbf{q}_{1}, \cdots, \mathbf{q}_{n}$.

5. $\% \quad x$ : initial guess. $b$ : right hand side vector.

6. \% max_it: maximum number of iterations. tol: error tolerance.

7. $\%$ kappa: (real number) minimization step controller:

8. $\%$

9. $\%$

10. $\%$ output:

11. $\%$

12. $\%$

13. $\%$

14. $\%$ zero, standard minimization

15. \% storage: positive, Sleijpen-van der Vorst minimization

16. $\%$

17.

18. $\quad N=\operatorname{size}(A, 2) ; n=\operatorname{size}(Q, 2)$;

19. $G=z \operatorname{eros}(N, n) ; W=z \operatorname{eros}(N, n) ; \quad \%$ initialize workspace for $\widetilde{\mathbf{g}}, \mathbf{w}$ and $c$

20. $\quad c=z \operatorname{eros}(1, n) ; \quad \%$ end initialization

21.

22. $\quad$ iter $=0 ; \operatorname{flag}=1 ; \operatorname{bnrm} 2=\operatorname{norm}(b)$;

23. if bnrm $2==0.0$, bnrm $2=1.0$; end

24. $\quad r=b-A * x ;$ err $=\operatorname{norm}(r) /$ bnrm 2 ;

25. if err $<$ tol, flag $=0$; return, end

26.

27. $\quad G(:, 1)=M \backslash r ; W(:, 1)=A * G(:, 1) ; c(1)=Q(:, 1)^{\prime} * W(:, 1)$;

28. if $c(1)==0$, flag $=-1$; return, end

29. $e=Q(:, 1)^{\prime} * r$;

30.

31. for $j=0:$ max_it

32. for $i=1: n-1$

33. $\quad$ alpha $=e / c(i)$;

34.

35.

$x=x+$ alpha $* G(:, i)$;

$r=r-\operatorname{alph} a * W(:, i)$;

36. $\quad \operatorname{err}=\operatorname{norm}(r) /$ bnrm 2 ;

37. $\quad$ iter $=$ iter +1 ;

38. $\quad$ if $\mathrm{err}<\mathrm{tol}$, flag $=0$; return, end

39. $\quad$ if iter $>=$ max_it, return, end

40.

41.

42.

43.

44.

45.

46.

47.

48.

49.

$e=Q(:, i+1)^{\prime} * r$

if $j>=1$

beta $=-e / c(i+1)$

$W(:, i+1)=r+$ beta $* W(:, i+1) ;$

$G(:, i+1)=\operatorname{beta} * G(:, i+1)$;

for $s=i+1: n-1$

beta $=-Q(:, s+1)^{\prime} * W(:, i+1) / c(s+1)$;

$W(:, i+1)=W(:, i+1)+$ beta $* W(:, s+1)$;

$G(:, i+1)=G(:, i+1)+\operatorname{beta} * G(:, s+1)$; 
50.

51.

52.

53.

54.

55.

56.

57.

58.

59.

60.

61.

62.

63.

64.

65.

66.

67.

68.

69.

70.

71.

72.

73 .

74.

75.

76.

77.

78.

79.

80.

81.

82.

83.

84.

85.

86.

87.

88.

89.

90.

91.

92.

93.

94.

95.

96.

97.

98.

end

$$
G(:, i+1)=(M \backslash W(:, i+1))+(1 / r h o) * G(:, i+1) ;
$$

else

end

$$
G(:, i+1)=M \backslash r
$$

$W(:, i+1)=A * G(:, i+1)$;

for $s=0: i-1$

$$
\begin{aligned}
& \text { beta }=-Q(:, s+1)^{\prime} * W(:, i+1) / c(s+1) ; \\
& W(:, i+1)=W(:, i+1)+\operatorname{beta} * W(:, s+1) ;
\end{aligned}
$$$$
G(:, i+1)=G(:, i+1)+\operatorname{beta} * G(:, s+1) ;
$$

end

$c(i+1)=Q(:, i+1)^{\prime} * W(:, i+1)$;

if $c(i+1)==0$, flag $=-1$; return, end

end

$$
\text { alpha }=e / c(n)
$$

$x=x+$ alpha $* G(:, n) ;$

$r=r-$ alpha $* W(:, n)$;

err $=\operatorname{norm}(r) /$ bnrm 2 ;

if err $<$ tol, flag $=0$; iter $=$ iter +1 ; return, end

$u_{-} t=M \backslash r ; z=A * u_{-} t ; r h o=z^{\prime} * z$;

if $r h o==0$, flag $=-1$; return, end

omega $=z^{\prime} * r$;

if omega $==0$, flag $=-1$; return, end

rho $=-$ omega/rho;

if kappa $>0$

$$
\begin{aligned}
& \text { omega }=\text { omega } /(\operatorname{norm}(z) * \text { norm }(r)) \\
& \text { abs_om }=\text { abs }(\text { omega }) \\
& \text { if } a b s \_o m<k a p p a \\
& \quad r h o=r h o * k a p p a / a b s \_o m ; \\
& \text { end }
\end{aligned}
$$


99. end

\section{A sample run of $\mathrm{ML}(n)$ BiCGstab}

1. $N=100 ; A=\operatorname{randn}(N) ; M=\operatorname{randn}(N) ; b=\operatorname{randn}(N, 1)$;

2. $n=10 ;$ kappa $=0.7 ;$ tol $=10^{-7} ;$ max_it $=3 * N$;

3. $Q=\operatorname{sign}(\operatorname{randn}(N, n)) ; x=z \operatorname{eros}(N, 1) ; Q(:, 1)=b-A * x$;

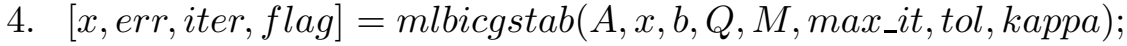

\section{ACKNOWLEDGEMENTS}

Thanks will be added.

\section{REFERENCES}

[1] J. Aliaga, D. Boley, R. Freund and V. Hernández, A Lanczos-type method for multiple starting vectors, Math. Comp. 69 (2000), pp. 1577-1601.

[2] A. Berman and R. J. Plemmons, Cones and iterative methods for best least squares solutions of linear systems, SIAM J. Numer. Anal., 11:145-154, 1974.

[3] A. Edelman, Eigenvalues and condition numbers of random matrices, SIAM J. Matrix Anal. Appl., 9(1988), 543-560.

[4] A. El Guennouni, K. JBilou And H. Sadok, A block version of BiCGSTAB for linear systems with multiple right-hand sides, ETNA 16 (2003), 129-142.

[5] R. Fletcher, Conjugate gradient methods for indefinite systems, volume 506 of Lecture Notes Math., pages 73-89. Springer-Verlag, Berlin-Heidelberg-New York, 1976.

[6] M. H. Gutknecht, A completed theory of the unsymmetric Lanczos process and related algorithms. Part I., SIAM J. Matrix Anal. Appl. 1992, 13:594-639.

[7] — - Variants of BICGStab for matrices with complex spectrum, SIAM J. Sci. Comput., 14 (1993), pp. 10201033.

[8] - A completed theory of the unsymmetric Lanczos process and related algorithms. Part II., SIAM J. Matrix Anal. Appl. 1994, 15:15-58.

[9] - Lanczos-type solvers for nonsymmetric linear systems of equations, Acta Numerica, 6 (1997), pp. $271-397$.

[10] - IDR Explained, submitted to ETNA.

[11] R. Horn And C. Johnson, Matrix analysis, Cambridge University Press, 1985.

[12] W. D. Joubert, Generalized conjugate gradient and Lanczos methods for the solution of nonsymmetric systems of linear equations, Ph.D. thesis and Tech. Report CNA-238, Center for Numerical Analysis, University of Texas, Austin, TX, 1990.

[13] - Lanczos methods for the solution of nonsymmetric systems of linear equations, SIAM Journal on Matrix Analysis and Applications 1992; 13:926-943.

[14] E. F. KaAsschieter, Preconditioned conjugate gradients for solving singular systems, Journal of Computational and Applied Mathematics 1988; 24:265-275.

[15] C. LAnczos, Solution of systems of linear equations by minimized iterations, J. Research Nat. Bureau of Standards, 49 (1952), pp. 33-53.

[16] I. MareK And D. B. Szyld, Comparison theorems for weak splittings of bounded operators, Numer. Math., 58:387-397, 1990.

[17] D. O'LEARY, The block conjugate gradient algorithm and related methods, Linear Algebra Appl., 29(1980), pp. 293-322.

[18] K. Moriya AND T. NoderA, Breakdown-free ML(k)BiCGStab algorithm for non-Hermitian linear systems, O. Gervasi et al. (Eds.): ICCSA 2005, LNCS 3483, pp. 978-988, 2005.

[19] L. REICHEL AND Q. YE, Breakdown-free GMRES for singular systems, SIAM Journal on Matrix Analysis and Applications 2005; 26:1001-1021.

[20] Y. SAAD, The Lanczos biorthogonalization algorithm and other oblique projection methods for solving large unsymmetric systems, SIAM Journal on Numerical Analysis, 19(1982), pp.485-506.

[21] — Iterative methods for sparse linear systems, 2nd edition, SIAM, Philadelphia, PA, 2003.

[22] Y. SAAD AND M. H. Schultz, GMRES: A generalized minimal residual algorithm for solving nonsymmetric linear systems, SIAM J. Sci. Statist. Comput., 7 (1986), pp. 856-869.

[23] Y. SAAD AND H. A. VAN DER VORST, Iterative solution of linear systems in the 20-th century, J. Comp. and Appl. Math., 123(1-2):1-33, 2000. 
[24] G. L. G. Sleijpen And D. R. Fokkema, BiCGSTAB(l) for linear equations involving unsymmetric matrices with complex spectrum, ETNA, 1:11-32, 1993.

[25] G. L.G. Sleijpen, P. Sonneveld, and M. B. van Gijzen, Bi-CGSTAB as an induced dimension reduction method, Preprint 1369, Dep. Math., University Utrecht (April, 2008).

[26] G. L. G. Sleijpen And H. A. VAn Der Vorst, Maintaining convergence properties of BiCGSTAB methods in finite precision arithmetic, Numer. Algorithms, 10 (1995), pp. 203-223.

[27] — Reliable updated residuals in hybrid Bi-CG methods, Computing, 56 (1996), pp. 141-163.

[28] G. L. G. Sleijpen, H. A. Van Der Vorst, and D. R. Fokkema, BiCGstab(l) and other hybrid Bi-CG methods, Numerical Algorithms, 7 (1994), pp. 75-109. Received Oct. 29, 1993.

[29] P. Sonneveld, CGS, a fast Lanczos-type solver for nonsymmetric linear systems, SIAM J. Sci. Statist. Comput., 10 (1989), pp. 36-52.

[30] P. Sonneveld And M. van Gijzen, IDR(s): a family of simple and fast algorithms for solving large nonsymmetric linear systems, Delft University of Technology, Reports of the Department of Applied Mathematical Analysis, Report 07-07.

[31] - IDR(s): a family of simple and fast algorithms for solving large nonsymmetric linear systems, SIAM J. Sci. Comput. Vol. 31, No. 2, pp. 1035-1062.

[32] H. A. VAN DER Vorst, Bi-CGSTAB: A fast and smoothly converging variant of Bi-CG for the solution of nonsymmetric linear systems, SIAM J. Sci. Statist. Comput., 12 (1992), pp. 631-644.

[33] - Iterative Krylov methods for large linear systems, Cambridge University Press, Cambridge, April 2003.

[34] H. VAn Der Vorst And Q. YE, Residual Replacement Strategies for Krylov Subspace Iterative Methods for the Convergence of True Residuals, SIAM J. Sci. Comput., 22 (2000):836-852.

[35] M. VAn GiJzen And P. Sonneveld, An elegant IDR(s) variant that efficiently exploits bi-orthogonality properties, Delft University of Technology, Reports of the Department of Applied Mathematical Analysis, Report 08-21.

[36] Y. WeI, H. Wu, Convergence properties of Krylov subspace methods for singular linear systems with arbitrary index, Journal of Computational and Applied Mathematics 2000; 114:305-318.

[37] P. Wesseling And P. Sonneveld, Numerical experiments with a multiple grid and a preconditioned Lanczos type method, in Approximation methods for Navier-Stokes problems (Proc. Sympos., Univ. Paderborn, Paderborn, 1979), vol. 771 of Lecture Notes in Math., Springer, Berlin, 1980, pp. 543-562.

[38] M. Yeung And D. Boley, Transpose-free multiple Lanczos and its application in Padé approximation, Journal of Computational and Applied Mathematics, Vol 177/1 pp. 101-127, 2005.

[39] M. Yeung And T. Chan, ML(k)BiCGSTAB: A BiCGSTAB variant based on multiple Lanczos starting vectors, SIAM J. Sci. Comput., Vol. 21, No. 4, pp. 1263-1290, 1999.

[40] N. ZhANG AND Y. WEI, On the convergence of general stationary iterative methods for range-Hermitian singular linear systems, Numer. Linear Algebra Appl. 2010; 17:139-154.

[41] Shao-Liang Zhang, GPBi-CG: Generalized product-type methods based on Bi-CG for solving nonsymmetric linear systems, SIAM J. Sci. Comput., 18:537-551, 1997. 


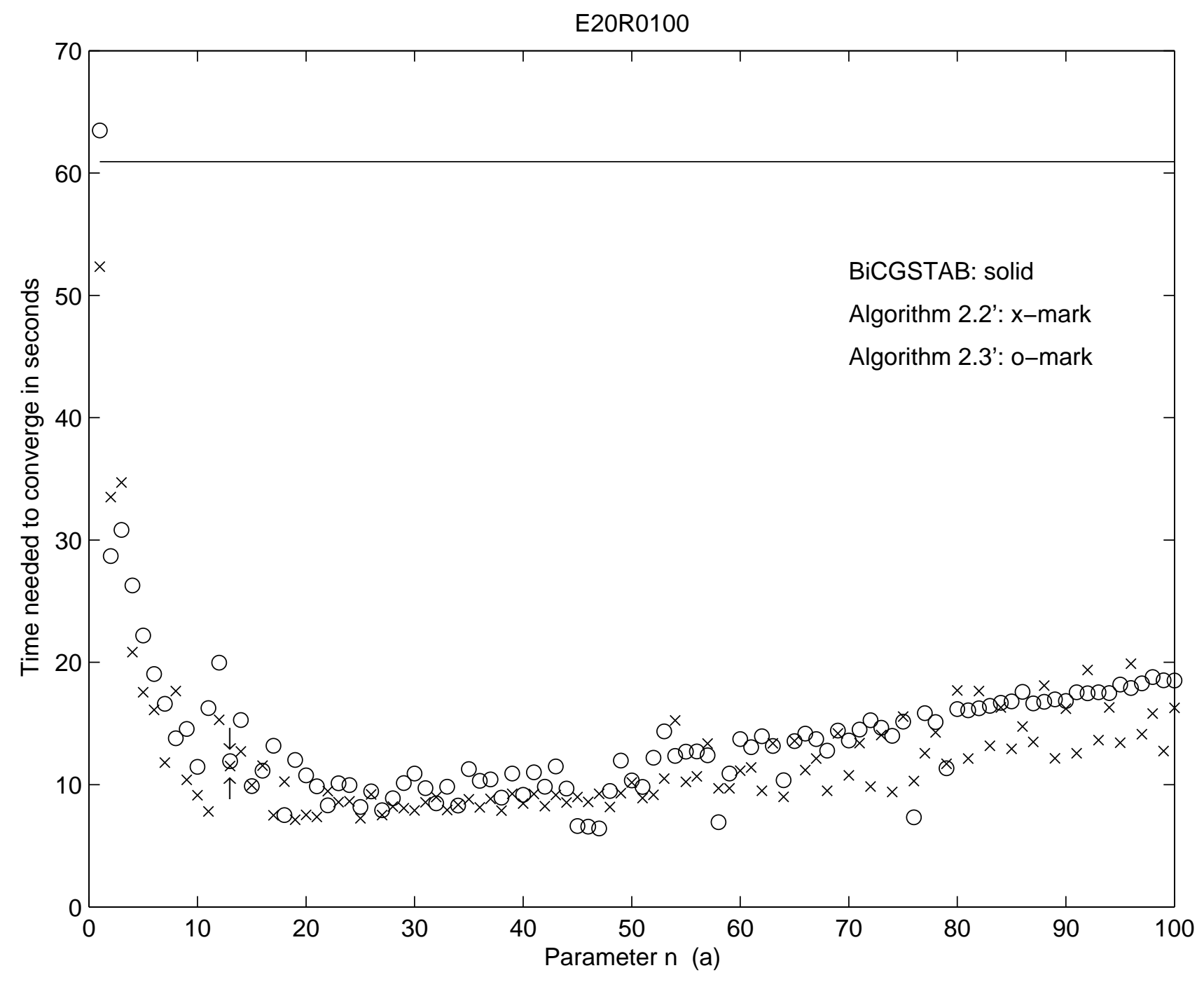




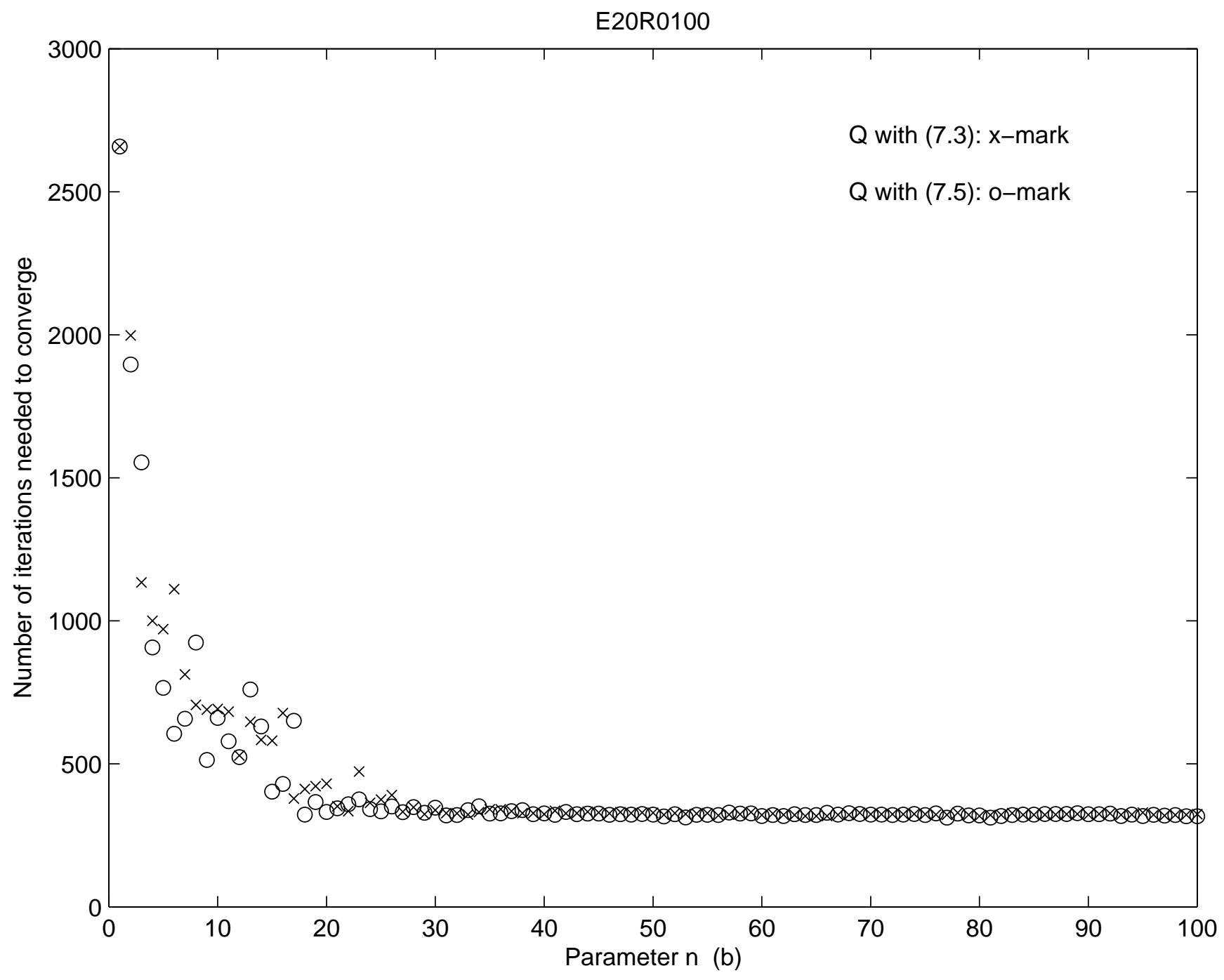




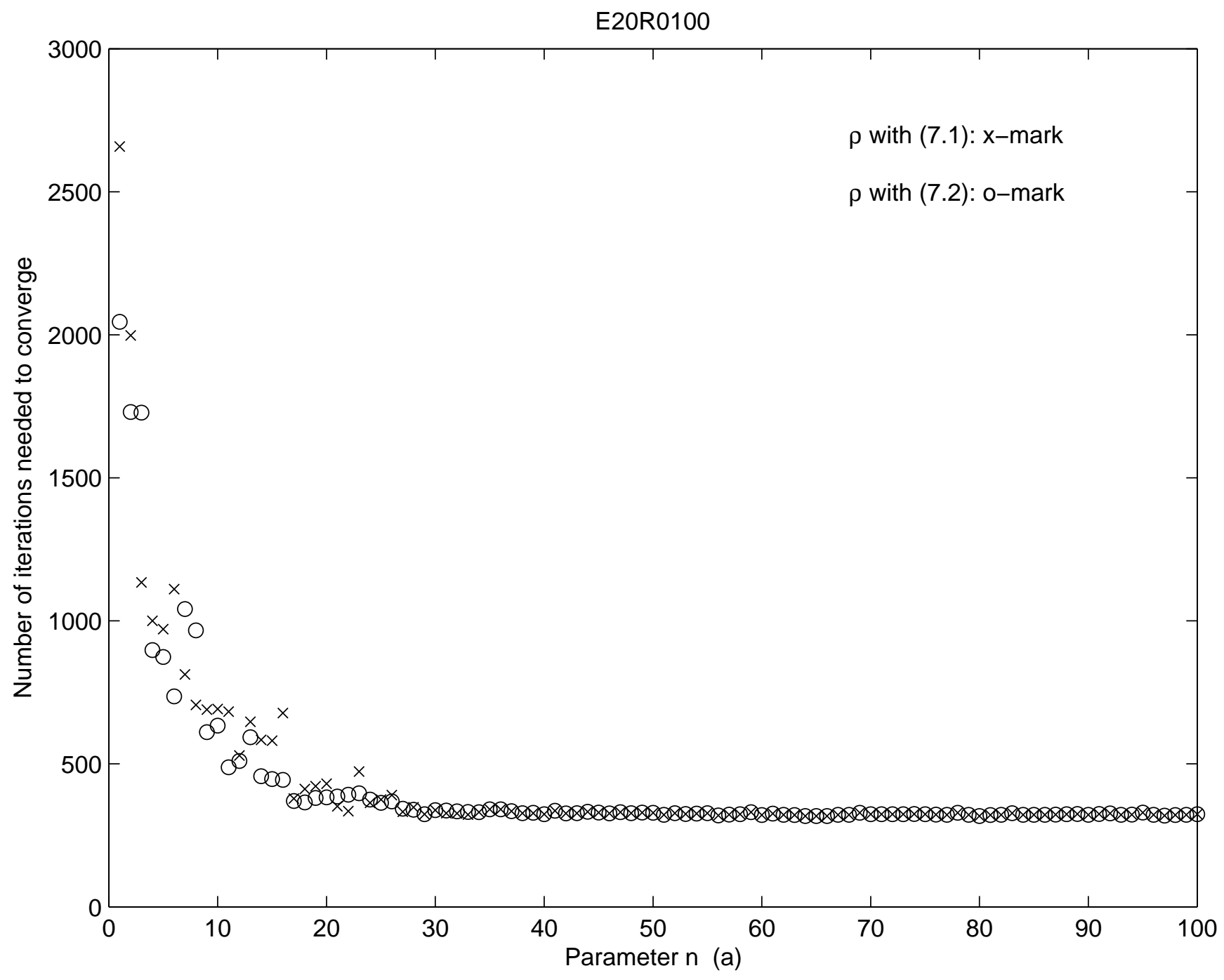




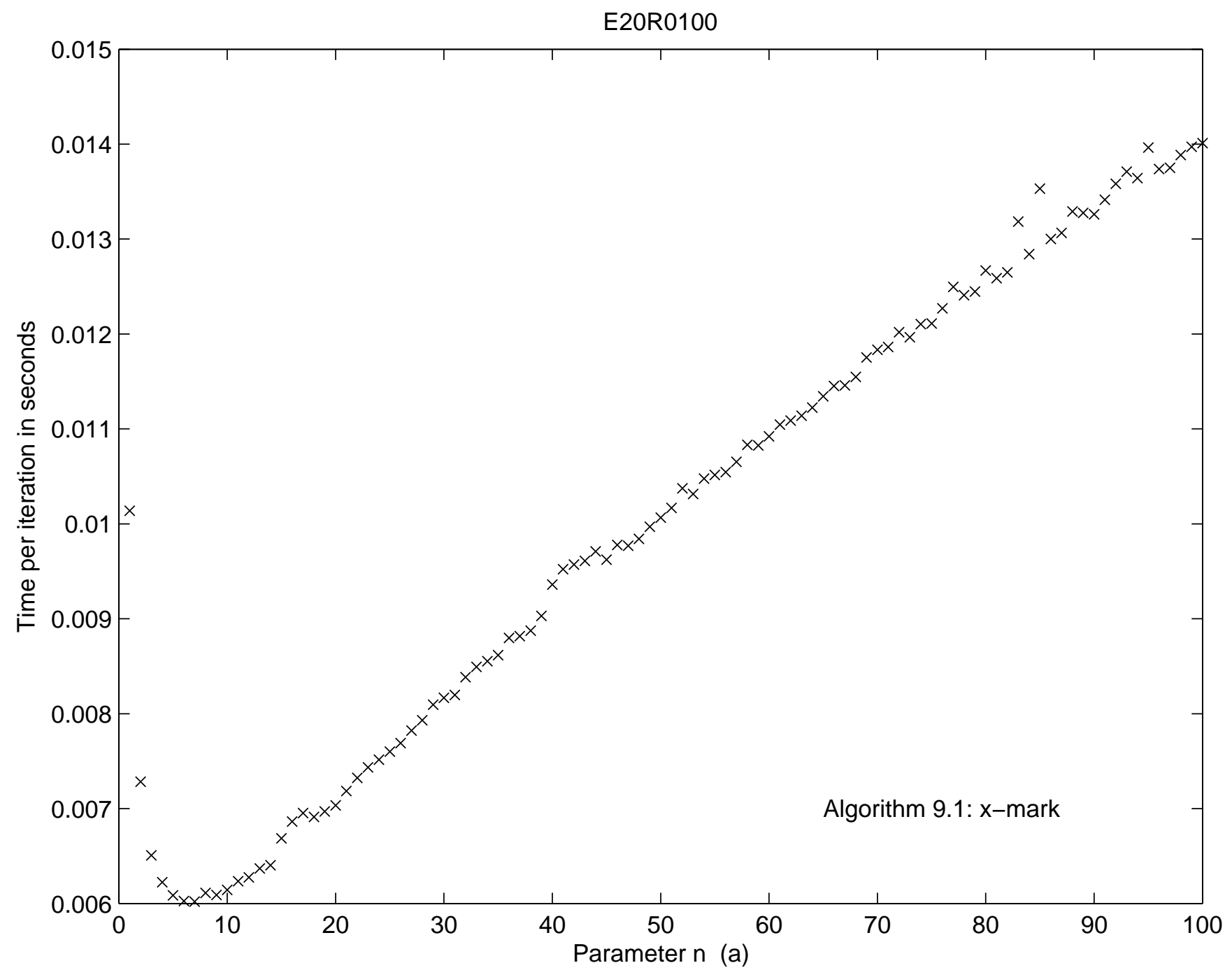




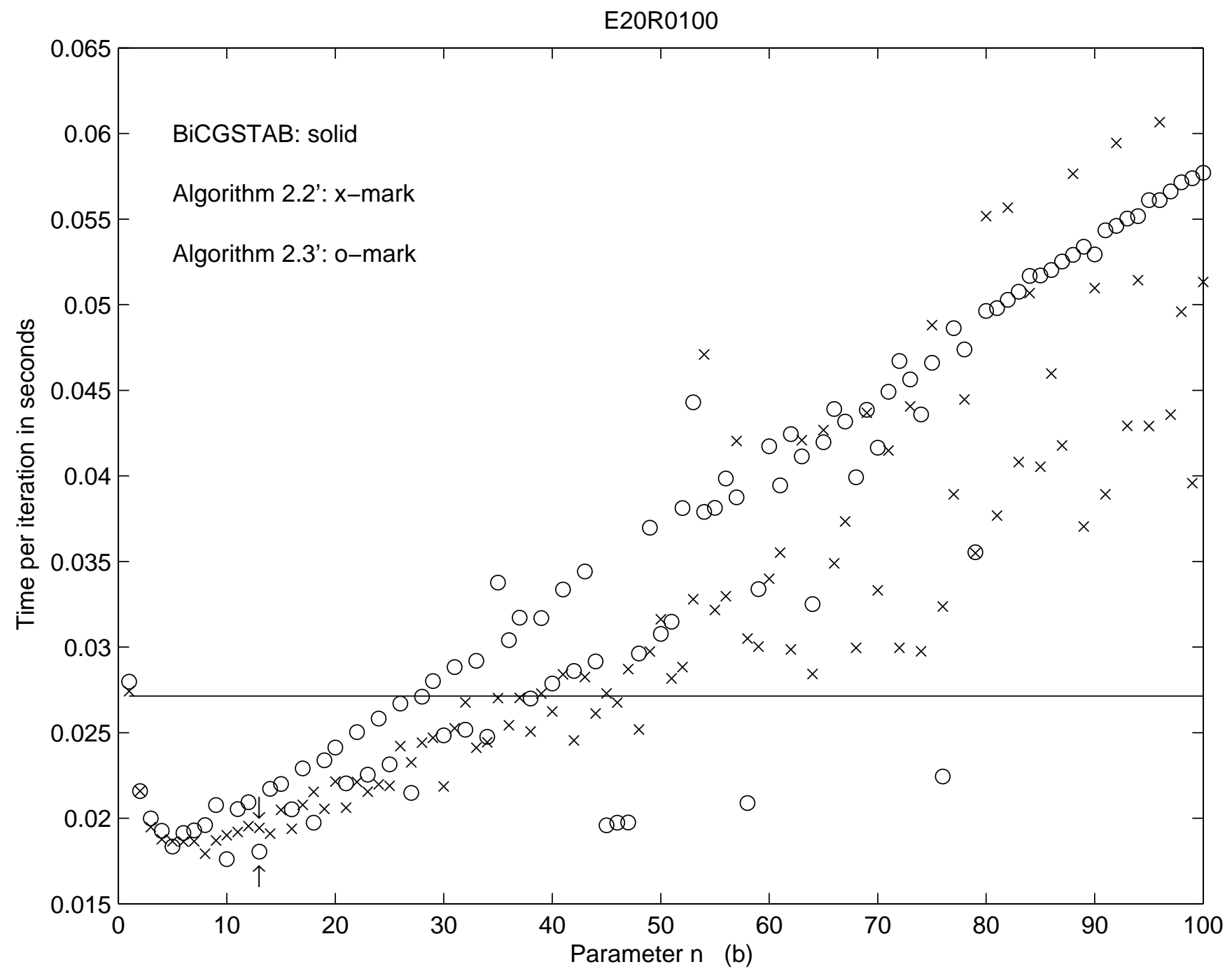




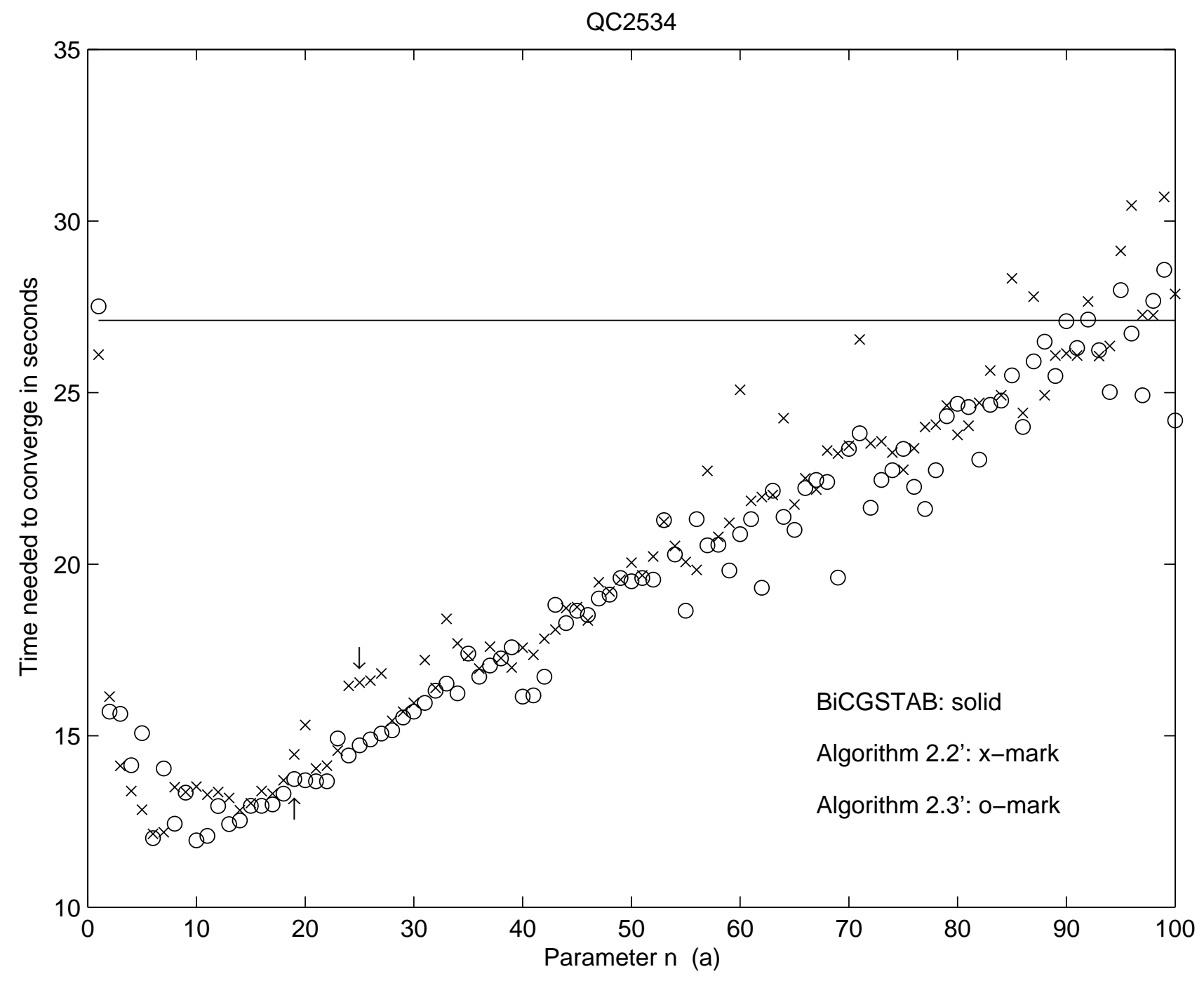




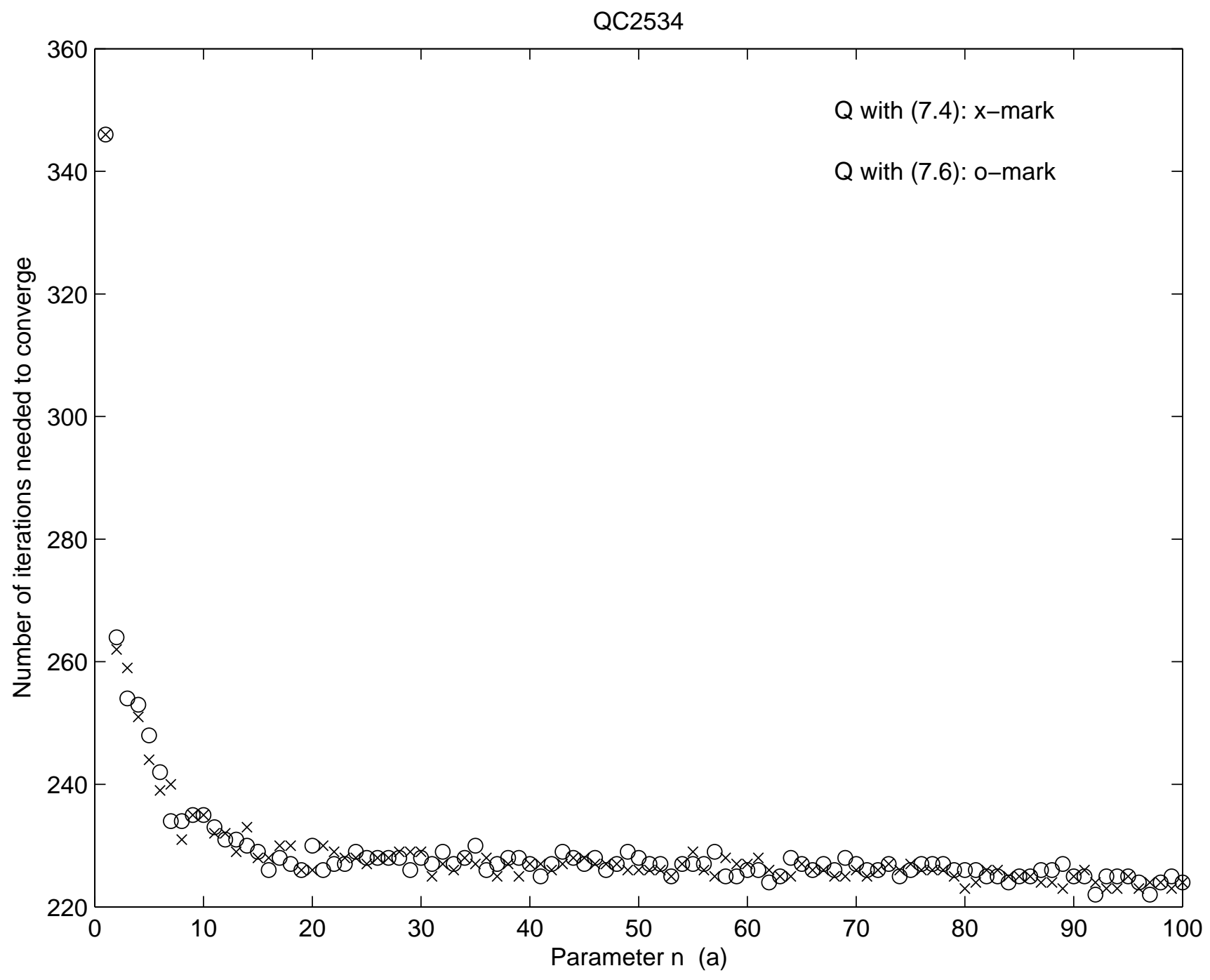




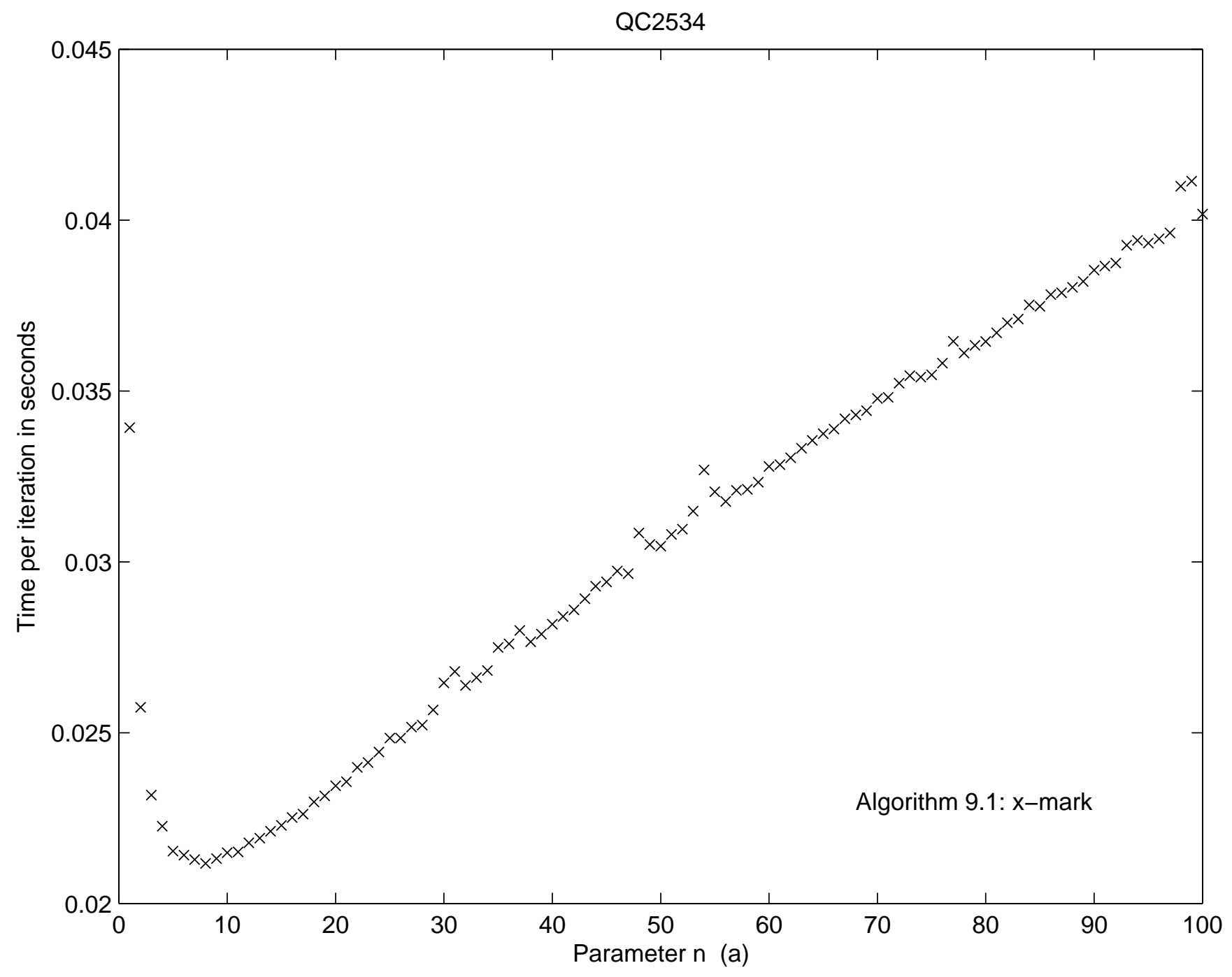




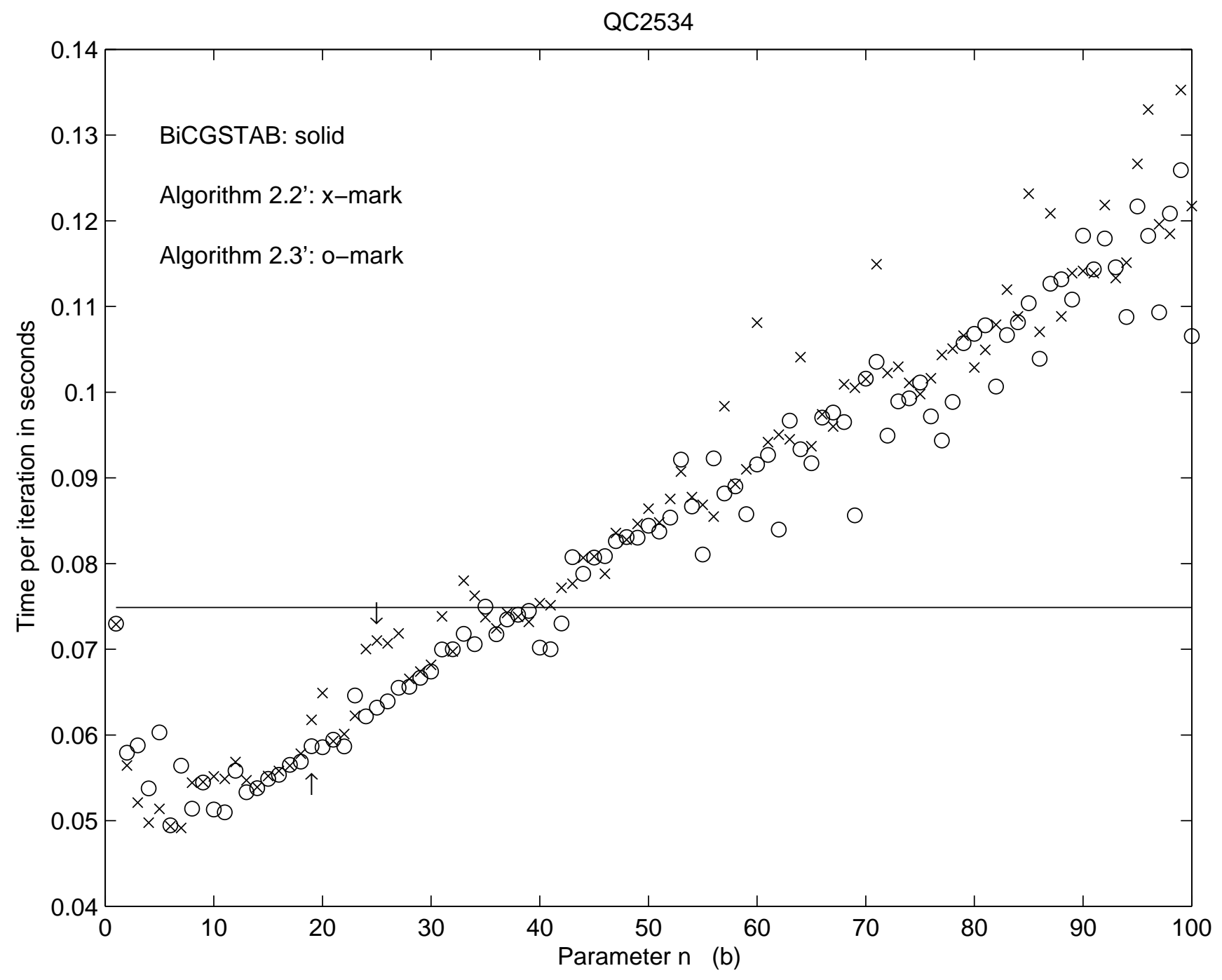




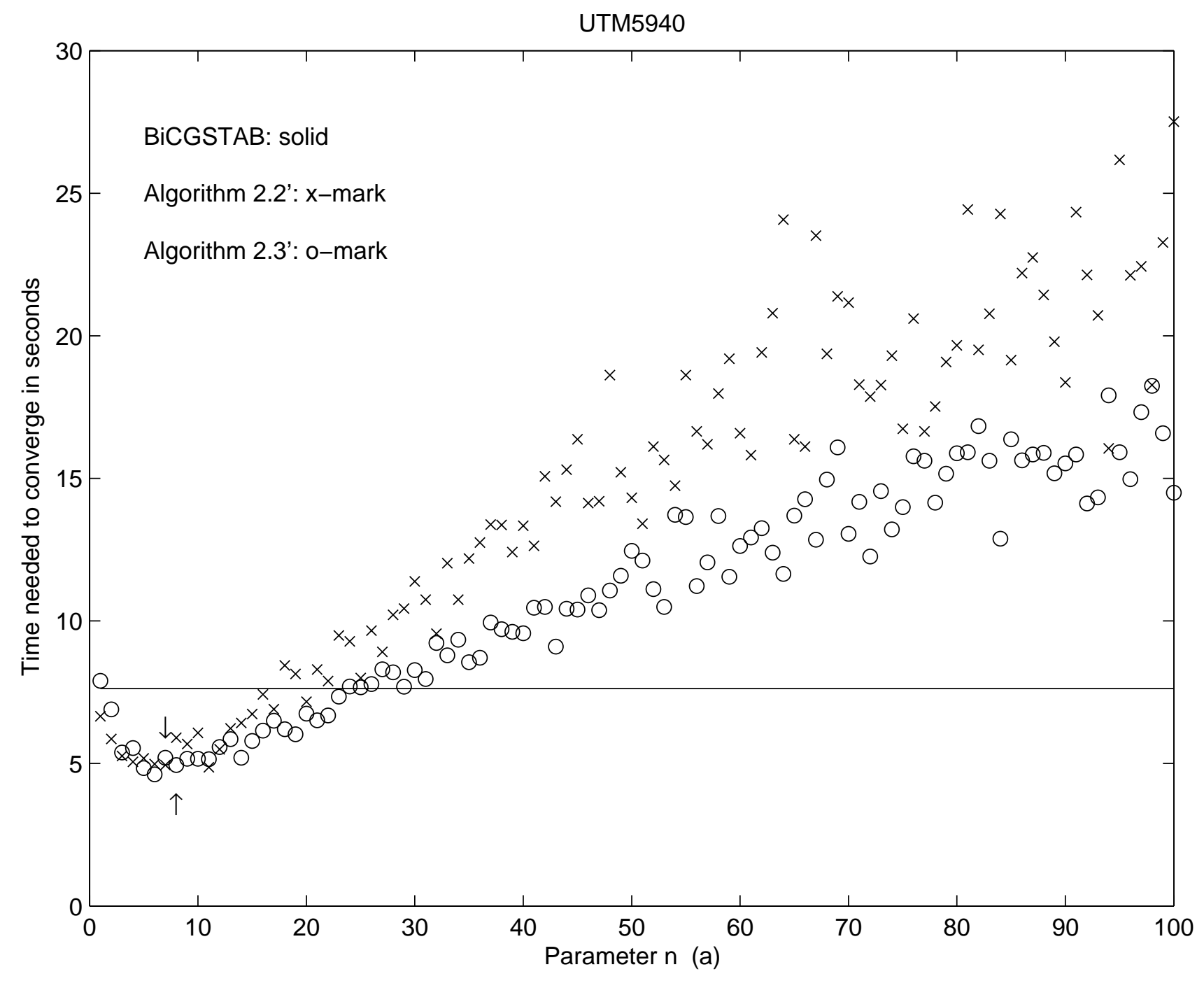




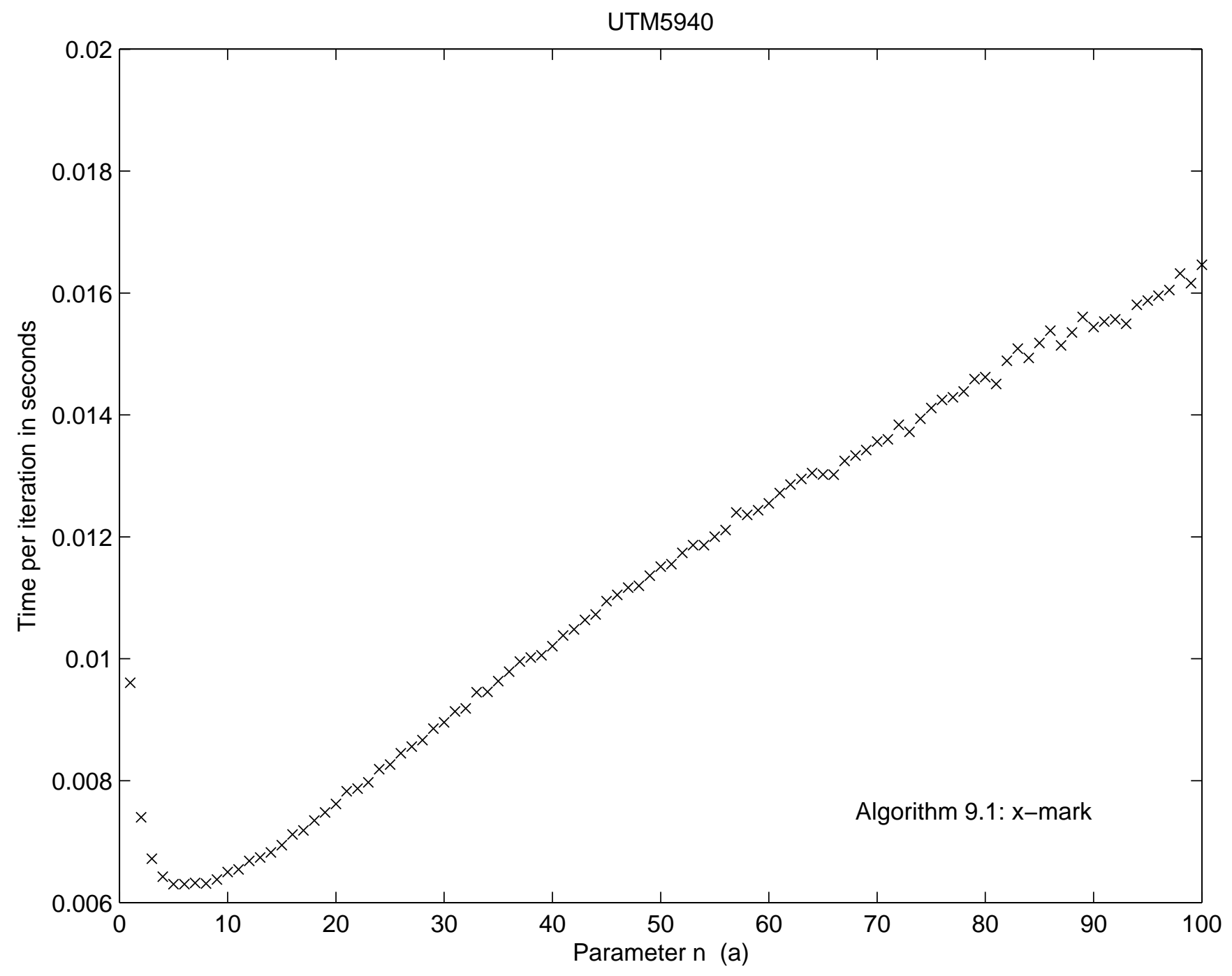




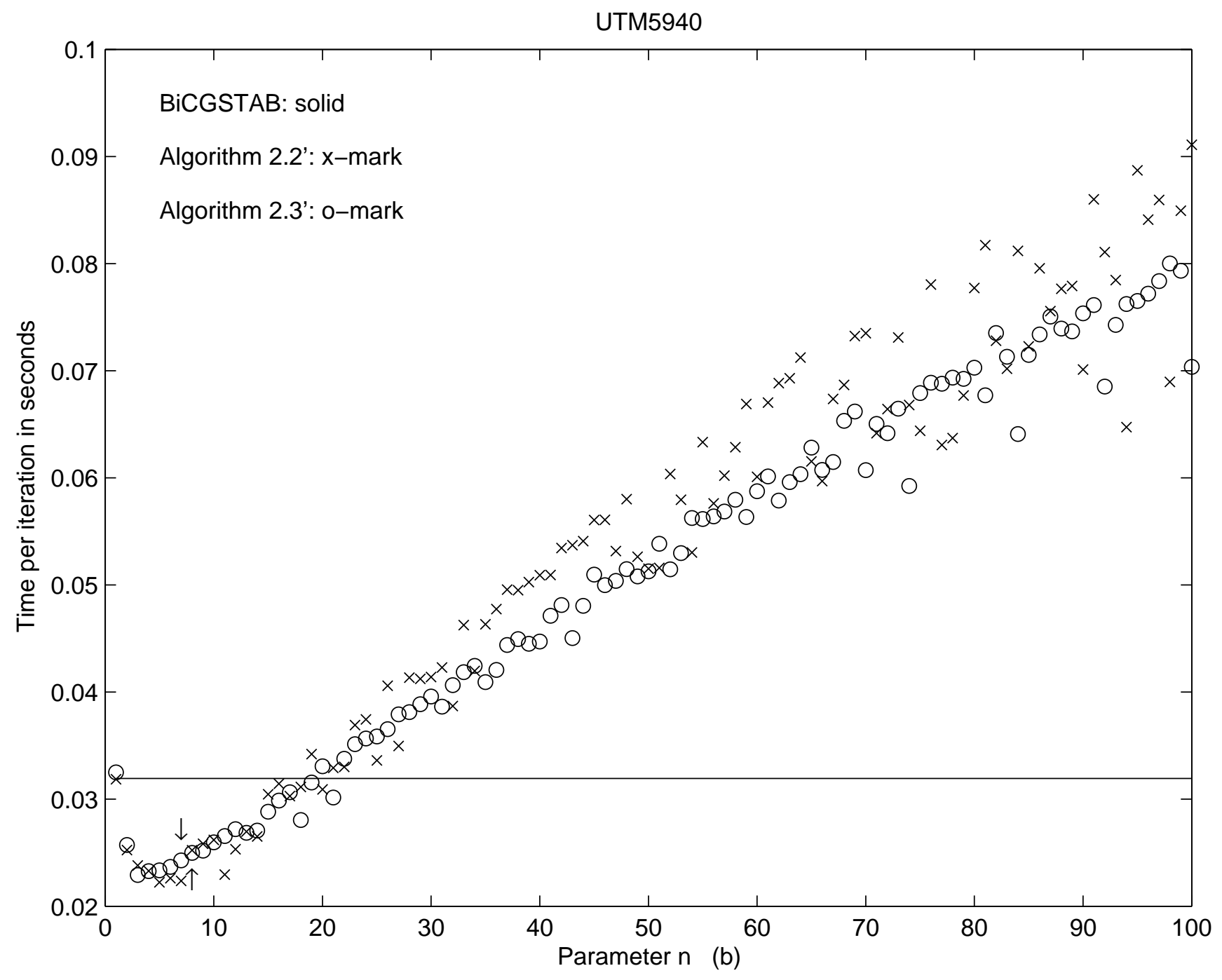

